\title{
Asymmetric Direct Vinylogous Michael Reaction of Activated Alkenes to Nitroolefins Catalyzed by Modified Cinchona Alkaloids
}

\author{
Dong Xue ${ }^{a, c}$, Ying-Chun Chen* ${ }^{b}$, Qi-Wei Wang, ${ }^{a}$ Ling-Feng Cun ${ }^{a}$, Jin Zhu ${ }^{a}$ and Jin-Gen Deng ${ }^{\text {a,c }}$ \\ ${ }^{a}$ Key Laboratory of Asymmetric Synthesis \& Chirotechnology of Sichuan Province and Union Laboratory of \\ Asymmetric Synthesis, Chengdu Institute of Organic Chemistry, Chinese Academy of Sciences, Chengdu \\ 610041, China; ${ }^{b}$ Department of Medicinal Chemistry, West China School of Pharmacy, Sichuan University, \\ Chengdu 610041, China; ${ }^{c}$ Graduated School of Chinese Academy of Sciences, Beijing, China \\ E-mail: jgdeng@cioc.ac.cn; ycchenhuaxi@yahoo.com.cn
}

\section{Supporting Information}

Table of Contents

1. General Methods........................................................................ 2

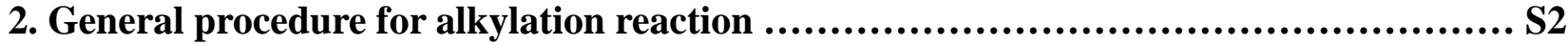

3. General procedure for asymmetric direct vinylogous Michael reaction ...................S3

4. The absolute configurations of the vinylogous Michael reaction product 3c..............S10

5. Preparation and transformation of enantiopure addition product ..................... S10

6. Crystal data of compounds, 2, rac-3e, 3c, and rac-5................................S13

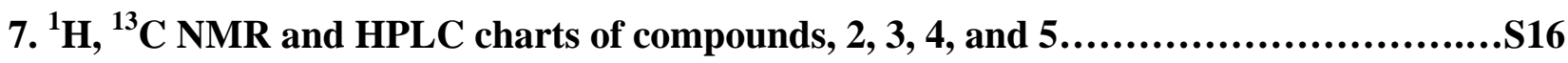


1. General Methods: Column chromatography was performed using silica gel (200-300 mesh) eluting with ethyl acetate and petroleum ether or menthol and Methylene chloride. TLC was performed on glass-backed silica plates. ${ }^{1} \mathrm{H}$ and ${ }^{13} \mathrm{C}$ NMR was recorded on Bruker 300 and 600 MHz spectrometers. Chemical shifts were reported in ppm down field from tetramethylsilane with the solvent resonance as the internal standard. HPLC analysis was carried on Waters-Breeze (2487 Dual Absorbance Detector and 1525 Binary HPLC Pump) using chiral columns. Chiralpak AS column was purchased from Daicel Chemical Industries, LTD. Optical rotations were measured on a Perkin-Elmer 341 Polarimeter at $=589 \mathrm{~nm}$. FT-ICRMS spectra were recorded on P-SIMS-Gly of Bruker Daltonics. Inc.. Melting points were determined in open caplillaries and were uncorrected. Commercial grade solvents were dried and purified by standard procedures as specified in Purification of Laboratory Chemicals, $4^{\text {th }}$ Ed (Armarego, W. L. F.; Perrin, D. D. Butterworth Heinemann: 1997). All catalytic reactions and manipulations were performed under argon.

\section{General procedure for alkylation reaction}<smiles>N#CC(C#N)=C1CCCc2ccccc21</smiles>
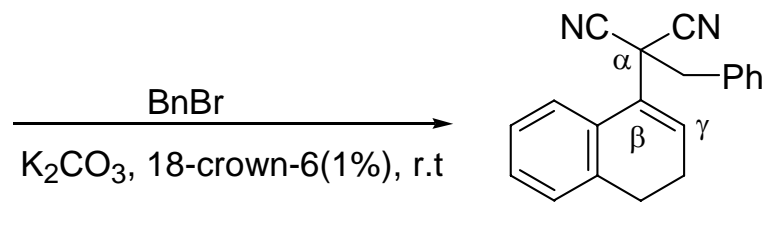

2h, Yield: 94\%

Benzyl bromide (90 mg, $0.64 \mathrm{mmol}$ ) and 18-crown-6 (1.4 mg, $0.0064 \mathrm{mmol})$ were added to a stirred mixture of activated olefin 1a $(103 \mathrm{mg}, 0.53 \mathrm{mmol})$ and $\mathrm{K}_{2} \mathrm{CO}_{3}(84 \mathrm{mg}, 0.61 \mathrm{mmol})$ in DCM $(0.5 \mathrm{~mL})$. The solution was stirred at room temperature and monitored by TLC. After $2 \mathrm{~h}$, the reaction was completed. The solution was concentrated and flash chromatography (ethyl acetate/petroleum ether $=1: 10$ ) gave the $\alpha$-alkylation product, 2 (Yield: 94\%).<smiles>N#CC(C#N)(Cc1ccccc1)C1=CCCc2ccccc21</smiles>

2-Benzyl-2-(3,4-dihydro-naphthalen-1-yl)malononitrile 2. White solid, mp: 78-80 ${ }^{\circ} ;{ }^{1} \mathrm{H} \mathrm{NMR}\left(\mathrm{CDCl}_{3}, 300 \mathrm{MHz}\right) \delta(\mathrm{ppm}) 7.81$ (d, $\left.J=8.0 \mathrm{~Hz}, 1 \mathrm{H}\right)$, 7.38-7.16 (m, 8H), 6.49 (t, $J=4.8 \mathrm{~Hz}, 1 \mathrm{H}), 3.62$ (s, 2H), 2.69-2.65 (m, 2H), 
2.34-2.27 (m, 2H); ${ }^{13} \mathrm{C} \mathrm{NMR}\left(\mathrm{CDCl}_{3}, 75 \mathrm{MHz}\right) \delta(\mathrm{ppm})$ 137.4, 133.4, 131.7, 130.3, 129.4, 128.6, 128.5, 128.4, 127.2, 126.7, 123.2, 114.8, 42.7, 42.5, 27.5, 23.2; IR (KBr): v 3432, 3060, 3032, 2955, 1497, 1485, 1448, 1231, 1089, 825, 769, 736, $698 \mathrm{~cm}^{-1}$; HRMS (Positive-SIMS) calcd for $\mathrm{C}_{20} \mathrm{H}_{16} \mathrm{~N}_{2}+$ Glycerol $+\mathrm{H}$ : 377.1865 , found: 377.1860 . The structure of alkylation product was further proved by X-ray crystallographic analysis (Figure 1).

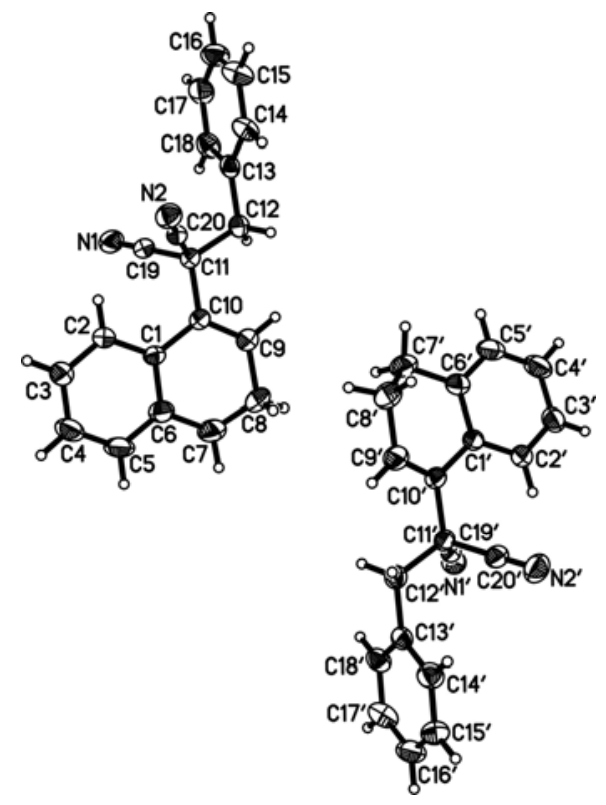

Figure 1 X-Ray crystallographic structure of alkylation product 2.

\section{General procedure for asymmetric direct vinylogous Michael reaction}

Modified cinchona alkaloid (3.0 mg, $0.0034 \mathrm{mmol})$, activated alkene $(0.068 \mathrm{mmol})$ and nitroolefin $(0.068 \mathrm{mmol})$ were added to a dry flask in turn. The flask was put into a cooler at -40 ${ }^{\circ} \mathrm{C}$, and freshly distilled $\mathrm{CH}_{2} \mathrm{Cl}_{2}(0.3 \mathrm{~mL})$ was added under argon. After the reaction was completed, the solution was concentrated and flash chromatography (ethyl acetate $=1$ : 10 ) gave the corresponding asymmetric vinylogous Michael addition product.

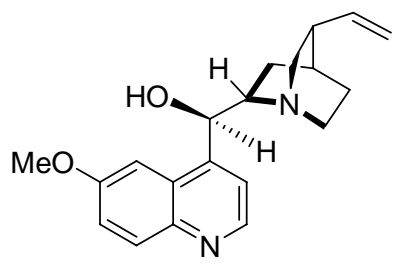

Quinine<smiles>C=CC1CN2CCC1C[C@H]2[C@H](O)c1ccnc2ccc(OC)cc12</smiles>

Quinidine 


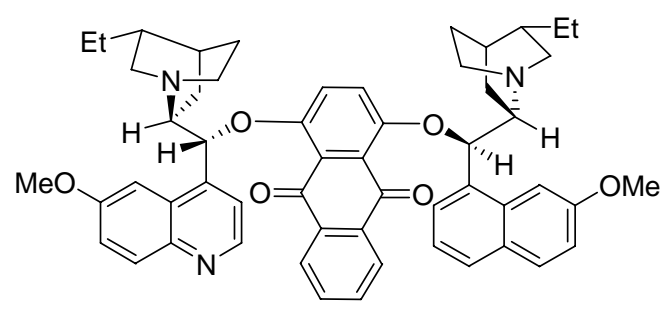

$[\mathrm{DHQ}]_{2} \mathrm{AQN}$

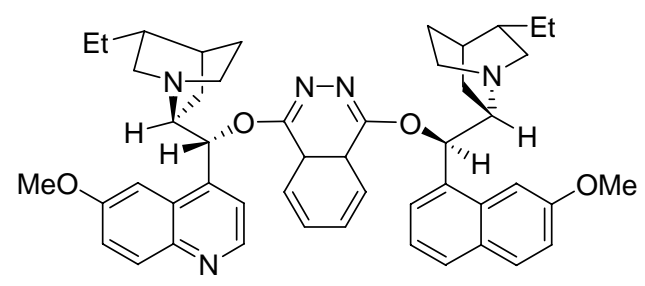

$[\mathrm{DHQ}]_{2} \mathrm{PHAL}$

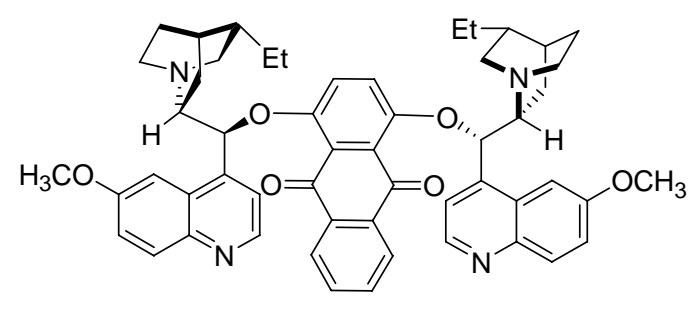

$[\mathrm{DHQD}]_{2} \mathrm{AQN}$

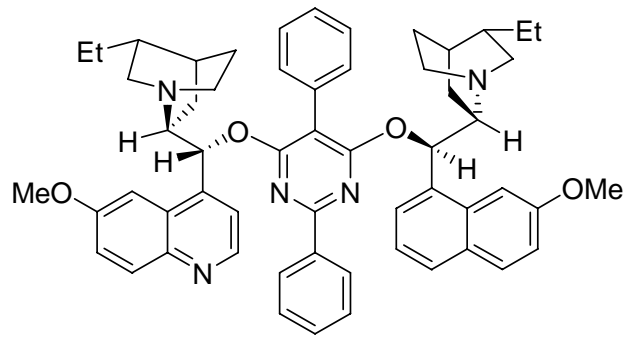

$[\mathrm{DHQ}]_{2} \mathrm{PYR}$<smiles>CCN1CC2CCC1C2[C@H](Oc1nc(-c2ccccc2)nc(OC(c2ccnc3ccc(OC)cc23)[C@@H]2CC3CCN2C3)c1-c1ccccc1)c1ccnc2ccc(OC)cc12</smiles>

$[D H Q D]_{2} P Y R$

Chart 1 The structure of organocatalysts. All modified cinchona alkaloids were purchased from Aldrich.<smiles>N#CC(C#N)=C1c2ccccc2CC[C@H]1[C@H](C[N+](=O)[O-])c1ccccc1</smiles>

2-[2-(1-Phenyl-2-nitroethyl)-3,4-dihydro-2H-naphalen-1-ylidene]-malon

onitrile (3a). White solid, mp: $163-165^{\circ} \mathrm{C} ;{ }^{1} \mathrm{H}$ NMR $\left(\mathrm{CDCl}_{3}, 300 \mathrm{MHz}\right) \delta$ (ppm) 8.07 (d, $J=7.8$ Hz, 1H), 7.62-7.57 (m, 1H), 7.44-7.33 (m, 5H), 7.28-7.26 (m, 1H), 4.69 (dd, $J=12.6,2.5$ Hz, 1H), 4.42 (dd, $J=12.6,5.1 \mathrm{~Hz}, 1 \mathrm{H}), 3.66-3.61$ (m, 1H), 3.51-3.45 (m, 1H), 3.03-2.99 (m, 1H), 2.92-2.89, 1.84-1.82 (m, 1H); ${ }^{13} \mathrm{C} \mathrm{NMR}\left(\mathrm{CDCl}_{3}, 75 \mathrm{MHz}\right) \delta$ (ppm) 174.6, 139.5, 135.9, 134.5, 130.0, 129.4, 128.7, 128.6, 127.8, 127.7, 127.3, 113.1, 78.6, 44.8, 44.5, 25.5, 24.0; IR (KBr): v 3369, 2227, 1457, 1381, 1155, $740 \mathrm{~cm}^{-1}$; HRMS (ESI) calcd for $\mathrm{C}_{21} \mathrm{H}_{17} \mathrm{~N}_{3} \mathrm{O}_{2}+\mathrm{NH}_{4}$ : 361.1659, found: 361.1665; 91\% ee determined by HPLC on AS column, 30\% 2-propanol/hexane, $1.0 \mathrm{ml} / \mathrm{min}, \mathrm{UV} 254 \mathrm{~nm}, \mathrm{t}_{\mathrm{minor}}=13.8 \mathrm{~min}, \mathrm{t}_{\mathrm{major}}=20.9 \mathrm{~min} ;[\alpha]_{\mathrm{D}}{ }^{25}-203.5^{\circ}$ (c 0.06, EtOAc). 
<smiles>Cc1ccc(C(CN[N+](=O)[O-])[C@H]2CCc3ccccc3C2=C(C#N)C#N)cc1</smiles>

\section{2-[2-(1-p-Tolyl-2-nitroethyl)-3,4-dihydro-2H-naphalen-1-ylidene]}

-malononitrile (3b). White solid, mp: $173-175{ }^{\circ} \mathrm{C}$; ${ }^{1} \mathrm{H}$ NMR $\left(\mathrm{CDCl}_{3}, 300 \mathrm{MHz}\right) \delta(\mathrm{ppm}) 8.06$ (d, $J=7.9 \mathrm{~Hz}, 1 \mathrm{H}), 7.59$ (t, $J=7.5 \mathrm{~Hz}, 1 \mathrm{H}), 7.44-7.13$ (m, 6H), 4.67 (dd, $J=12.5,10.4 \mathrm{~Hz}, 1 \mathrm{H}), 4.40$ (dd, $J=12.5,5.1 \mathrm{~Hz}, 1 \mathrm{H}$ ), 3.63-3.59 (m, 1H), 3.46-3.41 (m, 1H), 3.02-2.98 (m, 1H), 2.91-2.89 (m, 1H), 2.35 (s, 3H), 1.99-1.95 (m, 1H), 1.85-1.84 (m, 1H); ${ }^{13} \mathrm{C} \mathrm{NMR}\left(\mathrm{CDCl}_{3}, 75 \mathrm{MHz}\right) \delta(\mathrm{ppm})$ 174.7, 139.5, 138.6, 134.4, 132.8, 130.1, 129.9, 128.6, 127.8, 127.5, 127.3, 113.1, 80.9, 78.7, 44.8, 44.2, 25.5, 24.0, 21.1; IR (KBr): v 3442, 2922, 2226, 1554, 1513, 1456, 1379, 813, 773, $737 \mathrm{~cm}^{-1}$; HRMS (SIMS) calcd for $\mathrm{C}_{22} \mathrm{H}_{19} \mathrm{~N}_{3} \mathrm{O}_{2}+$ Glycerol+H: 450.2023, found: 450.2016; 90\% ee was determined by HPLC on AS column, 30\% 2-propanol/hexane, $1.0 \mathrm{ml} / \mathrm{min}$, UV 254nm, $\mathrm{t}_{\mathrm{minor}}=11.2$ $\min , \mathrm{t}_{\text {major }}=17.9 \mathrm{~min} ;[\alpha]_{\mathrm{D}}{ }^{25}-189.4^{\circ}\left(\mathrm{c} 0.06, \mathrm{CHCl}_{3}\right)$<smiles>N#CC(C#N)=C1c2ccccc2CC[C@H]1[C@H](C[N+](=O)[O-])c1ccc(Cl)cc1</smiles>

2-\{2-[1-(p-Chloro-phenyl)-2-nitroethyl]-3,4-dihydro-2H-naphalen-1ylidene\}-malononitrile (3c). White solid, mp: $197-199{ }^{\circ} \mathrm{C} ;{ }^{1} \mathrm{H} \mathrm{NMR}\left(\mathrm{CDCl}_{3}, 300 \mathrm{MHz}\right) \delta(\mathrm{ppm})$ 8.08 (dd, $J=7.9,1.0 \mathrm{~Hz}, 1 \mathrm{H}), 7.58-7.61$ (m, 1H), 7.42-7.33 (m, 4H), 7.22 (dd, $J=2.4,6.6 \mathrm{~Hz}$, 2H), 4.67 (dd, $J=12.7,10.4 \mathrm{~Hz}, 1 \mathrm{H}$ ), 4.40 (dd, $J=12.7,4.9 \mathrm{~Hz}, 1 \mathrm{H}$ ), 3.61-3.56 (m, $1 \mathrm{H}$ ), 3.49-3.43 (m, $1 \mathrm{H}), 2.93-2.91(\mathrm{~m}, 2 \mathrm{H}), 2.04-1.98(\mathrm{~m}, 1 \mathrm{H}), 1.82-1.80(\mathrm{~m}, 1 \mathrm{H})$; ${ }^{13} \mathrm{C} \mathrm{NMR}\left(\mathrm{CDCl}_{3}\right.$, $75 \mathrm{MHz}) \delta(\mathrm{ppm}) 174.0,139.3,134.8,134.7,134.5,130.1,129.7,129.1,128.7,127.7,127.5$, 113.1, 112.9, 81.2, 78.4, 44.5, 43.9, 25.4, 23.9; IR (KBr): v 3469, 2229, 1574, 1557 1494, 1379, 1093, 1013, 825, 771, $741 \mathrm{~cm}^{-1}$; HRMS (ESI) calcd for $\mathrm{C}_{21} \mathrm{H}_{16} \mathrm{ClN}_{3} \mathrm{O}_{2}+\mathrm{Na}$ : 400.0529, found: 400.0644; 91\% ee was determined by HPLC on AS column, 30\% 2-propanol/hexane, $1.0 \mathrm{ml} / \mathrm{min}$, UV 254nm, $\mathrm{t}_{\text {minor }}=16.5 \mathrm{~min}, \mathrm{t}_{\text {major }}=22.6 \mathrm{~min} ;[\alpha]_{\mathrm{D}}{ }^{25}-221.7^{\circ}\left(c \mathrm{cos}, \mathrm{CHCl}_{3}\right)$.<smiles>Cc1ccc(C(C[N+](=O)[O-])[C@H]2CCc3ccccc3C2=C(C#N)C#N)cc1</smiles>

Br 2-\{2-[1-(p-Bromo-phenyl)-2-nitro-ethyl]-3,4-dihydro-2H-naphalen-1ylidene\}-malononitrile (3d). White solid, mp: 195-197 ${ }^{\circ} \mathrm{C} ;{ }^{1} \mathrm{H} \mathrm{NMR}\left(\mathrm{CDCl}_{3}, 300 \mathrm{MHz}\right) \delta(\mathrm{ppm})$ 8.08 (dd, $J=7.9,0.83 \mathrm{~Hz}, 1 \mathrm{H}), 7.51-7.61$ (m, 3H), 7.42-7.33 (m, 1H), 7.34 (d, $J=6.6 \mathrm{~Hz}, 1 \mathrm{H})$, $7.14(\mathrm{~d}, J=6.6 \mathrm{~Hz}, 2 \mathrm{H}), 4.66$ (dd, $J=12.8,10.4 \mathrm{~Hz}, 1 \mathrm{H}), 4.39$ (dd, $J=12.7,4.9 \mathrm{~Hz}, 1 \mathrm{H})$, 
3.61-3.56 (m, 1H), 3.48-3.43 (m, 1H), 2.96-2.92 (m, 2H), 1.99-1.98 (m, 1H), 1.83-1.81 (m, 1H); ${ }^{13} \mathrm{C} \mathrm{NMR}\left(\mathrm{CDCl}_{3}, 75 \mathrm{MHz}\right) \delta(\mathrm{ppm}) 173.9,139.3,134.9,134.6,132.7,130.1,129.4,128.7,127.7$, 127.5, 122.8, 113.0, 112.9, 78.3, 77.4, 44.4, 43.9, 25.4, 23.9; IR (KBr): v 3464, 2927, 2229, 1574, 1557, 1494, 1436, 1379, 1093, 1013, 824, 770, 740, $651 \mathrm{~cm}^{-1}$; HRMS (SIMS) calcd for $\mathrm{C}_{21} \mathrm{H}_{16} \mathrm{BrN}_{3} \mathrm{O}_{2}+$ Glycerol+H: 514.0972, found: 514.0972; 91\% ee was determined by HPLC on AS column, 30\% 2-propanol/hexane, $1.0 \mathrm{ml} / \mathrm{min}$, UV 254nm, $\mathrm{t}_{\text {minor }}=17.1 \mathrm{~min}, \mathrm{t}_{\mathrm{major}}=24.2 \mathrm{mmin} ;[\alpha]_{\mathrm{D}}{ }^{25}$ $-198.8^{\circ}\left(\right.$ c $\left.0.27, \mathrm{CHCl}_{3}\right)$.<smiles>COc1ccc(C(C[N+](=O)[O-])C2CCc3ccccc3C2=C(C#N)C#N)cc1</smiles>

2-\{2-[1-(4-Methoxy-phenyl)-2-nitro-ethyl]-3,4-dihydro-2H-napht halen-1-ylidene\}-malononitrile (3e). White solid, mp: $181-183^{\circ} \mathrm{C} ;{ }^{1} \mathrm{H} \mathrm{NMR}\left(\mathrm{CDCl}_{3}, 300 \mathrm{MHz}\right) \delta$ (ppm) 8.07 (dd, $J=7.9,0.83 \mathrm{~Hz}, 1 \mathrm{H}), 7.59-7.57$ (m, 1H), 7.41-7.32 (m, 2H), 7.18 (d, $J=6.7 \mathrm{~Hz}$, 2H), 6.90 (d, $J=6.6 \mathrm{~Hz}, 2 \mathrm{H}$ ), 4.65 (dd, $J=12.5,10.4 \mathrm{~Hz}, 1 \mathrm{H}), 4.39$ (dd, $J=12.5,5.1 \mathrm{~Hz}, 1 \mathrm{H}$ ), 3.80 (s, 3H), 3.61-3.56 (m, 1H), 3.45-3.40 (m, 1H), 2.91-2.89 (m, 2H), 1.98-1.96 (m, 1H), 1.86-1.84 (m, 1H); ${ }^{13} \mathrm{C} \mathrm{NMR}\left(\mathrm{CDCl}_{3}, 75 \mathrm{MHz}\right) \delta(\mathrm{ppm})$ 174.8, 159.7, 139.5, 134.5, 130.0, 128.8, 128.6, 127.8, 127.6, 127.3, 114.8, 113.1, 113.0, 80.9, 78.8, 55.3, 44.9, 43.9, 25.5, 24.0; IR (KBr): $v$ 3469, 2229, 1573, 1557, 1514, 1379, 1258, 1178, 1035, 827, 772, 740, $574 \mathrm{~cm}^{-1}$; HRMS (ESI) calcd for $\mathrm{C}_{22} \mathrm{H}_{19} \mathrm{~N}_{3} \mathrm{O}_{3}+\mathrm{NH}_{4}$ : 391.1765, found: 391.1772; 94\% ee was determined by HPLC on AS column, 30\% 2-propanol/hexane, $1.0 \mathrm{ml} / \mathrm{min}, \mathrm{UV} 254 \mathrm{~nm}, \mathrm{t}_{\text {minor }}=18.0 \mathrm{~min}, \mathrm{t}_{\mathrm{major}}=41.1 \mathrm{mmin}$; $[\alpha]_{\mathrm{D}}{ }^{25}-172.3^{\circ}\left(\mathrm{c} \quad 0.17, \mathrm{CHCl}_{3}\right)$. The anti-stereochemistry of $3 \mathbf{e}$ was proved by X-ray crystallographic analysis of racemic $3 \mathbf{e}$ (Figure 2).

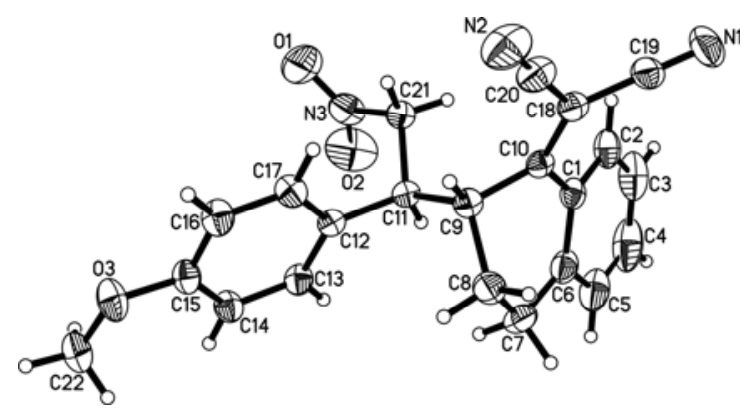

Figure 2 X-Ray crystallographic structure of rac-3e. 
<smiles>CN(C)c1ccc(C(C[N+](=O)[O-])C2(C=C(C#N)C#N)CCc3ccccc32)cc1</smiles>

\section{2-\{2-[1-(4-Dimethylamino-phenyl)-2-nitro-ethyl]-3,4-dihydro-2}

$\boldsymbol{H}$-naphthalen-1-ylidene\}-malononitrile (3f). White solid, mp: $195-197^{\circ} \mathrm{C} ;{ }^{1} \mathrm{H} \mathrm{NMR}\left(\mathrm{CDCl}_{3}\right.$, $300 \mathrm{MHz}) \delta(\mathrm{ppm}) 8.07$ (d, $J=7.9 \mathrm{~Hz}, 1 \mathrm{H}), 7.61-7.55$ (m, 1H), 7.43-7.32 (m, 2H), 7.09 (d, $J=8.1$ Hz, 2H), 6.69 (d, $J=8.1 \mathrm{~Hz}, 2 \mathrm{H}), 4.68-4.61$ (m, 1H), 4.38 (dd, $J=12.3,5.2 \mathrm{~Hz}, 1 \mathrm{H}), 3.59-3.55$ (m, 1H), 3.39-3.31 (m, 1H), 3.06-3.02 (m, 1H), 2.96 (bs, 6H), 2.89-2.81 (m, 1H), 2.01-1.91(m, 2H); ${ }^{13} \mathrm{C} \mathrm{NMR}\left(\mathrm{CDCl}_{3}, 75 \mathrm{MHz}\right) \delta$ (ppm) 175.2, 150.2, 139.7, 134.4, 130.0, 128.7, 128.4, 127.9, 127.2, 113.2, 112.9, 80.7, 78.9, 45.1, 43.9, 40.4, 25.7, 24.1; IR (KBr): v 3469, 2228, 1615, 1561, 1526, 1382, 1360, 1168, 945, 813, 773, $740 \mathrm{~cm}^{-1}$; HRMS (ESI) calcd for $\mathrm{C}_{23} \mathrm{H}_{22} \mathrm{~N}_{4} \mathrm{O}_{2}+\mathrm{H}: 387.1816$, found: 387.1823; 93\% ee was determined by HPLC on AS column, 30\% 2-propanol/hexane, $1.0 \mathrm{ml} / \mathrm{min}, \mathrm{UV} 254 \mathrm{~nm}, \mathrm{t}_{\mathrm{minor}}=18.1 \mathrm{~min}, \mathrm{t}_{\mathrm{major}}=39.9 \mathrm{mmin} ;[\alpha]_{\mathrm{D}}^{25}-109.1^{\circ}\left(c 0.15, \mathrm{CHCl}_{3}\right)$.<smiles>N#CC(C#N)=C1c2ccccc2CC[C@H]1c1ccco1</smiles>

2-[2-(1-Furan-2-yl-2-nitro-ethyl)-3,4-dihydro-2H-naphthalen-1-ylidene]malononitrile (3g). White solid, mp: $145-147^{\circ} \mathrm{C} ;{ }^{1} \mathrm{H} \mathrm{NMR}\left(\mathrm{CDCl}_{3}, 300 \mathrm{MHz}\right) \delta(\mathrm{ppm}) 7.99$ (d, $J$ = 7.9 Hz, $1 \mathrm{H})$, 7.59-7.57 (m, 1H), 7.40-7.30 (m, 3H), 6.33-6.26 (m, 2H), 4.79-4.72 (m, 1H), 4.42-4.38 (m, 1H), 3.71-3.68 (m, 2H), 3.04-2.91 (m, 2H), 2.08-2.04 (m, 1H), 1.86-1.84 (m, 1H); ${ }^{13} \mathrm{C} \mathrm{NMR}\left(\mathrm{CDCl}_{3}, 75 \mathrm{MHz}\right) \delta(\mathrm{ppm}) 173.9,148.7,143.2,139.5,134.3,129.8,128.6,127.9,127.2$, 113.0, 112.8, 110.5, 109.7, 81.5, 76.2, 43.2, 38.7, 25.5, 24.4; IR (KBr): v 2243, 1558, 1377, 1026, 774, 742, 731, $598 \mathrm{~cm}^{-1}$; HRMS (ESI) calcd for $\mathrm{C}_{19} \mathrm{H}_{15} \mathrm{~N}_{3} \mathrm{O}_{3}+\mathrm{Na}$ : 356.1006, found: 356.1007; 91\% ee was determined by HPLC on AS column, 30\% 2-propanol/hexane, $1.0 \mathrm{ml} / \mathrm{min}, \mathrm{UV} 254 \mathrm{~nm}$, $\mathrm{t}_{\text {minor }}=15.3$ min, $\mathrm{t}_{\text {major }}=23.8 \mathrm{~min} ;[\alpha]_{\mathrm{D}}^{25}-256.3^{\circ}\left(c 0.25, \mathrm{CHCl}_{3}\right)$.<smiles>N#CC(C#N)=C1c2ccccc2CC[C@H]1c1cccs1</smiles>

2-[2-(1-Thiophen-2-yl-2-nitroethyl)-3,4-dihydro-2H-naphthalen-1-yliden e]-malononitrile (3h). White solid, mp: $166-168^{\circ} \mathrm{C} ;{ }^{1} \mathrm{H} \mathrm{NMR}\left(\mathrm{CDCl}_{3}, 300 \mathrm{MHz}\right) \delta$ (ppm) 8.02 (d, $J=7.9$ Hz, 1H), 7.59-7.56 (m, 1H), 7.39-7.29 (m, 3H), 7.00-6.99 (m, 2H), 4.66 (dd, $J=12.7,9.9$ Hz, 1H), 4.44 (dd, $J=12.7,5.2$ Hz, 1H), 3.86-3.83 (m, 1H), 3.62-3.57 (m, 1H), 3.07-2.93 (m, 2H), 2.06-2.00 (m, 2H); ${ }^{13} \mathrm{C} \mathrm{NMR}\left(\mathrm{CDCl}_{3}, 75 \mathrm{MHz}\right) \delta(\mathrm{ppm})$ 173.9, 139.5, 138.1, 134.5, 129.9, 128.6, 
127.8, 127.4, 127.1, 125.9, 112.9, 81.3, 79.1, 45.8, 40.1, 25.5, 24.2; IR (KBr): v 3432, 2227, 1573, 1558, 1433, 1379, 775, 740, $699 \mathrm{~cm}^{-1}$; HRMS (ESI) calcd for $\mathrm{C}_{19} \mathrm{H}_{15} \mathrm{~N}_{3} \mathrm{O}_{2} \mathrm{~S}+\mathrm{NH}_{4}$ : 367.1223, found: 367.1224; 94\% ee was determined by HPLC on AS column, 30\% 2-propanol/hexane, $1.0 \mathrm{ml} / \mathrm{min}, \mathrm{UV} 254 \mathrm{~nm}, \mathrm{t}_{\text {minor }}=25.5 \mathrm{~min}, \mathrm{t}_{\text {major }}=40.9 \mathrm{~min} ;[\alpha]_{\mathrm{D}}{ }^{25}-239.8^{\circ}\left(c 0.11, \mathrm{CHCl}_{3}\right)$.

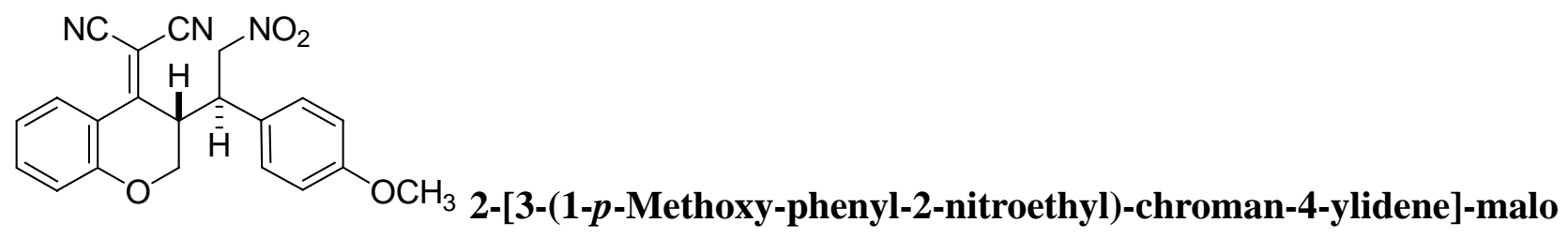
nonitrile (3i). Yellow solid, mp: $187-189^{\circ} \mathrm{C} ;{ }^{1} \mathrm{H}$ NMR $\left(\mathrm{CDCl}_{3}, 300 \mathrm{MHz}\right) \delta$ (ppm) 8.25 (d, $J=8.2$ Hz, 1H), 7.63-7.58 (m, 1H), 7.22 (d, $J=8.4 \mathrm{~Hz}, 2 \mathrm{H}), 7.17-7.12(\mathrm{~m}, 1 \mathrm{H}), 7.05$ (d, $J=8.4 \mathrm{~Hz}, 1 \mathrm{H})$, 6.93 (d, $J=8.2 \mathrm{~Hz}, 2 \mathrm{H}), 4.84-4.76$ (m, 1H), 4.47 (dd, $J=12.8,5.1 \mathrm{~Hz}, 1 \mathrm{H}), 4.15-4.06$ (m, 2H), 3.81 (s, 3H), 3.72-3.63 (m, 1H), 3.26 (d, $J=11.3 \mathrm{~Hz}, 1 \mathrm{H}) ;{ }^{13} \mathrm{C} \mathrm{NMR}\left(\mathrm{CDCl}_{3}, 75 \mathrm{MHz}\right) \delta(\mathrm{ppm})$ 165.4, 159.9, 156.0, 137.6, 129.2, 127.8, 126.9, 122.3, 118.5, 114.9, 114.8, 113.1, 112.7, 77.8, 77.6, 66.5, 55.3, 43.1, 42.9; IR (KBr): v 3430, 2226, 1610, 1567, 1556, 1514, 1481, 1383, 1331, 1256, 1034, 826, $746 \mathrm{~cm}^{-1}$; HRMS (ESI) $\mathrm{C}_{21} \mathrm{H}_{17} \mathrm{~N}_{3} \mathrm{O}_{4}+$ Glycerol+H: 468.1765, found: 468.1762; 70\% ee was determined by HPLC on AS column, 30\% 2-propanol/hexane, $1.0 \mathrm{ml} / \mathrm{min}$, UV 254nm, $\mathrm{t}_{\mathrm{minor}}=$ $22.18 \mathrm{~min}, \mathrm{t}_{\mathrm{major}}=47.3 \mathrm{mmin} ;[\alpha]_{\mathrm{D}}{ }^{25}-172.3^{\circ}\left(c 0.15, \mathrm{CHCl}_{3}\right)$.<smiles>N#CC(C#N)=C1c2ccccc2SCC1[C@H](C[N+](=O)[O-])c1ccccc1</smiles>

2-[3-(1-Phenyl-2-nitroethyl)-thiochroman-4-ylidene]-malononitrile (3j). Yellow solid, mp: $148-150{ }^{\circ} \mathrm{C} ;{ }^{1} \mathrm{H} \mathrm{NMR}\left(\mathrm{CDCl}_{3}, 300 \mathrm{MHz}\right) \delta(\mathrm{ppm}) 7.95$ (dd, $J=7.9,0.92 \mathrm{~Hz}$, 1H), 7.51-7.48 (m, 1H), 7.42-7.24 (m, 7H), 4.73 (dd, $J=12.6,9.7$ Hz, 1H), 4.36 (dd, $J=12.6,4.8$ $\mathrm{Hz}, 1 \mathrm{H}), 3.84-3.71$ (m, 2H), 3.34 (dd, $J=14.0,3.3 \mathrm{~Hz}, 1 \mathrm{H}), 2.64(\mathrm{dd}, J=14.0,3.2 \mathrm{~Hz}, 1 \mathrm{H}) ;{ }^{13} \mathrm{C}$ NMR $\left(\mathrm{CDCl}_{3}, 75 \mathrm{MHz}\right) \delta(\mathrm{ppm})$ 170.3, 137.9, 135.3, 134.5, 130.4, 129.5, 129.4, 129.3, 129.2, 129.0, 127.9, 127.4, 125.3, 124.3, 112.7, 112.5, 82.9, 77.9, 43.4, 41.6, 28.9; IR (KBr): v 3460, 2228, 1557, 1458, 1432, 1378, 1316, 907, 762, 739, 699 cm-1; HRMS (ESI) $\mathrm{C}_{20} \mathrm{H}_{15} \mathrm{~N}_{3} \mathrm{O}_{2} \mathrm{~S}+\mathrm{Na}$ : 384.0777, found: 384.0765; 86\% ee was determined by HPLC on AS column, 35\% 2-propanol/hexane, $1.0 \mathrm{ml} / \mathrm{min}, \mathrm{Uv} 254 \mathrm{~nm}, \mathrm{t}_{\mathrm{minor}}=15.78 \mathrm{~min}, \mathrm{t}_{\mathrm{major}}=31.04 \mathrm{~min} ;[\alpha]_{\mathrm{D}}{ }^{25}-686.4^{\circ}(c$ $\left.0.29 \mathrm{CHCl}_{3}\right)$. 
<smiles>COc1ccc(C(C[N+](=O)[O-])[C@H](C)C(=C(C#N)C#N)c2ccccc2)cc1</smiles>

\section{2-[3-(4-Methoxy-phenyl)-2-methyl-4-nitro-1-phenyl-butylidene]-}

malononitrile (3k). White solid, mp: $150-152^{\circ} \mathrm{C}$; ${ }^{1} \mathrm{H} \mathrm{NMR}\left(\mathrm{CDCl}_{3}, 300 \mathrm{MHz}\right) \delta(\mathrm{ppm})$ 7.61-7.56 (m, 3H), 7.36-7.33 (m, 2H), 7.10 (d, $J=8.6 \mathrm{~Hz}, 2 \mathrm{H}), 6.87$ (d, $J=8.6 \mathrm{~Hz}, 2 \mathrm{H}), 4.74-4.67$ (m, 1H), 4.62-4.57 (m, 1H), 3.78 (s, 3H), 3.53-3.50 (m, 2H), 0.98 (d, $J=6.1 \mathrm{~Hz}, 3 \mathrm{H}) ;{ }^{13} \mathrm{C} \mathrm{NMR}\left(\mathrm{CDCl}_{3}, 75\right.$ MHz) $\delta$ (ppm); 181.7, 159.6, 133.2, 131.5, 129.5, 129.1, 127.4, 126.9, 114.7, 111.5, 111.4, 89.2, 78.8, 55.2, 46.7, 44.8, 17.8; IR (KBr): v 3430, 2229, 1613, 1556, 1515, 1378, 1258, 1181, 1032, 831, $702 \mathrm{~cm}^{-1}$; HRMS (ESI) calcd for $\mathrm{C}_{21} \mathrm{H}_{19} \mathrm{~N}_{3} \mathrm{O}_{3}+\mathrm{NH}_{4}$ : 379.1765, found: 379.1773; 81\% ee was determined by HPLC on AS column, 30\% 2-propanol/hexane, $1.0 \mathrm{ml} / \mathrm{min}$, Uv 254nm, $\mathrm{t}_{\mathrm{minor}}=16.4$ $\min , \mathrm{t}_{\text {major }}=21.6 \mathrm{~min} ;[\alpha]_{\mathrm{D}}{ }^{25}+27.4^{\circ}\left(c 0.22, \mathrm{CHCl}_{3}\right)$.<smiles>C[C@H](C(=C(C#N)C#N)c1ccccc1)C(C[N+](=O)[O-])c1ccccc1</smiles>

2-(4-Nitro-1, 3-diphenyl-butylidene)-malononitrile (3l). White solid, mp: 88-90 ${ }^{\circ}$; ${ }^{1} \mathrm{H}$ NMR $\left(\mathrm{CDCl}_{3}, 300 \mathrm{MHz}\right) \delta$ (ppm) 7.61-7.57 (m, 3H), 7.38-7.32 (m, 5H), 7.21-7.18 (m, 2H), 4.79-4.72 (m, 1H), 4.63 (dd, $J=12.4,3.3 \mathrm{~Hz}, 1 \mathrm{H}), 3.59-3.56$ (m, 2H), 0.99 (d, $J=6.2 \mathrm{~Hz}, 3 \mathrm{H}) ;{ }^{13} \mathrm{C} \mathrm{NMR}\left(\mathrm{CDCl}_{3}, 75 \mathrm{MHz}\right) \delta(\mathrm{ppm})$ 181.4, 135.7, 133.2, 131.5, 129.5, 129.3, 128.6, 127.9, 126.9, 111.5, 89.3, 78.6, 47.3, 44.5, 17.8; IR (KBr): v 3461, 2230, 1552, 1494, 1454, 1377, 759, $700 \mathrm{~cm}^{-1}$; 76\% ee was determined by HPLC on AS column, 30\% 2-propanol/hexane, $1.0 \mathrm{ml} / \mathrm{min}, \mathrm{Uv} 254 \mathrm{~nm}, \mathrm{t}_{\text {minor }}=12.9 \mathrm{~min}, \mathrm{t}_{\mathrm{major}}=15.6 \mathrm{~min} ;[\alpha]_{\mathrm{D}}{ }^{25}+15.5^{\circ}\left(c 0.20, \mathrm{CHCl}_{3}\right)$.

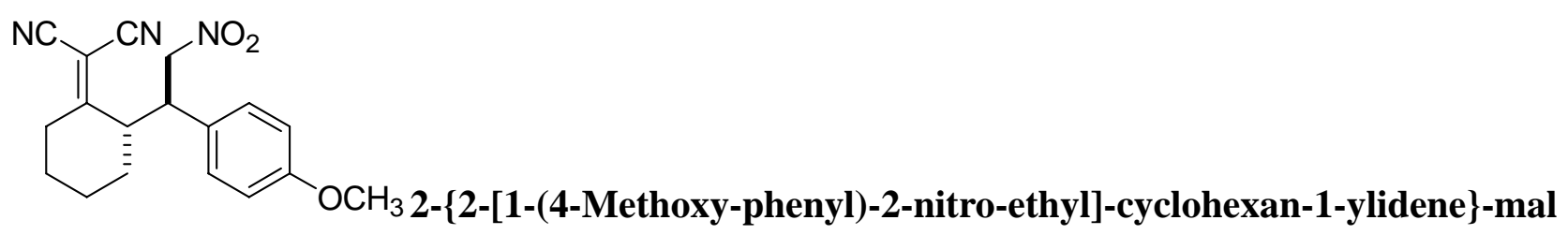

ononitrile (3m). White solid; mp: $146-148{ }^{\circ} \mathrm{C}$; ${ }^{1} \mathrm{H}$ NMR $\left(\mathrm{CDCl}_{3}, 300 \mathrm{MHz}\right) \delta(\mathrm{ppm}) 7.16(\mathrm{~d}, J=$ $8.7 \mathrm{~Hz} 2 \mathrm{H}$ ), 6.90 (d, $J=8.7 \mathrm{~Hz} 2 \mathrm{H}$ ), 4.58 (dd, $J=12.4,10.8 \mathrm{~Hz}, 1 \mathrm{H}$ ), 4.30 (dd, $J=12.4,4.8 \mathrm{~Hz}$, $1 \mathrm{H})$, 3.83-3.79 (m, 1H), $3.78(\mathrm{~s}, 3 \mathrm{H}), 3.32-3.31(\mathrm{~m}, 1 \mathrm{H}), 3.09-3.04(\mathrm{~m}, 1 \mathrm{H}), 2.58(\mathrm{~m}, 1 \mathrm{H})$, 2.15-2.30 (m, 1H), 1.69-1.51 (m, 5H); ${ }^{13} \mathrm{C}$ NMR $\left(\mathrm{CDCl}_{3}, 75 \mathrm{MHz}\right) \delta(\mathrm{ppm}) ; 185.0,159.6,128.8$, 127.4, 114.8, 111.3, 110.8, 84.9, 78.6, 55.2, 46.1, 44.0, 31.3, 30.1, 28.6, 19.3; HRMS (ESI) calcd for $\mathrm{C}_{18} \mathrm{H}_{19} \mathrm{~N}_{3} \mathrm{O}_{3}+\mathrm{NH}_{4}$ : 343.1765, found: 343.1762; $66 \%$ ee was determined by HPLC on AS column, 30\% 2-propanol/hexane, $1.0 \mathrm{ml} / \mathrm{min}, \mathrm{Uv} 254 \mathrm{~nm}, \mathrm{t}_{\text {minor }}=11.2 \mathrm{~min}, \mathrm{t}_{\text {major }}=42.4 \mathrm{~min} ;[\alpha]_{\mathrm{D}}{ }^{25}$ 
$-17.4^{\circ}\left(\right.$ c $\left.0.12, \mathrm{CHCl}_{3}\right)$.

\section{The absolute configurations of the vinylogous Michael reaction product 3c}

Enantiopure compound 3c (>99\% ee) were obtained by crystallized slowly from a mixture of ethyl acetate and petroleum ether and the crystals were suitable for X-ray structural analysis. Thus, the stereochemistry of the two newly created stereocenters of $3 \mathbf{c}$ was revealed to possess (10S,11R)-configuration (Figure 3).
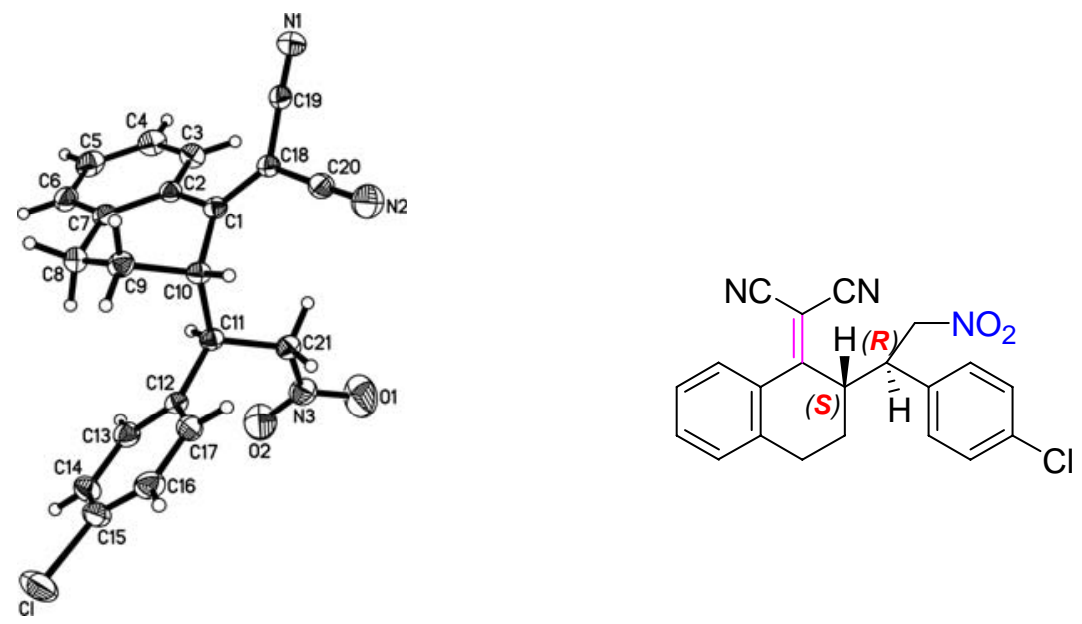

Figure 3 X-Ray crystallographic structure of enantiopure 3c.

\section{Preparation and transformation of enantiopure addition product}

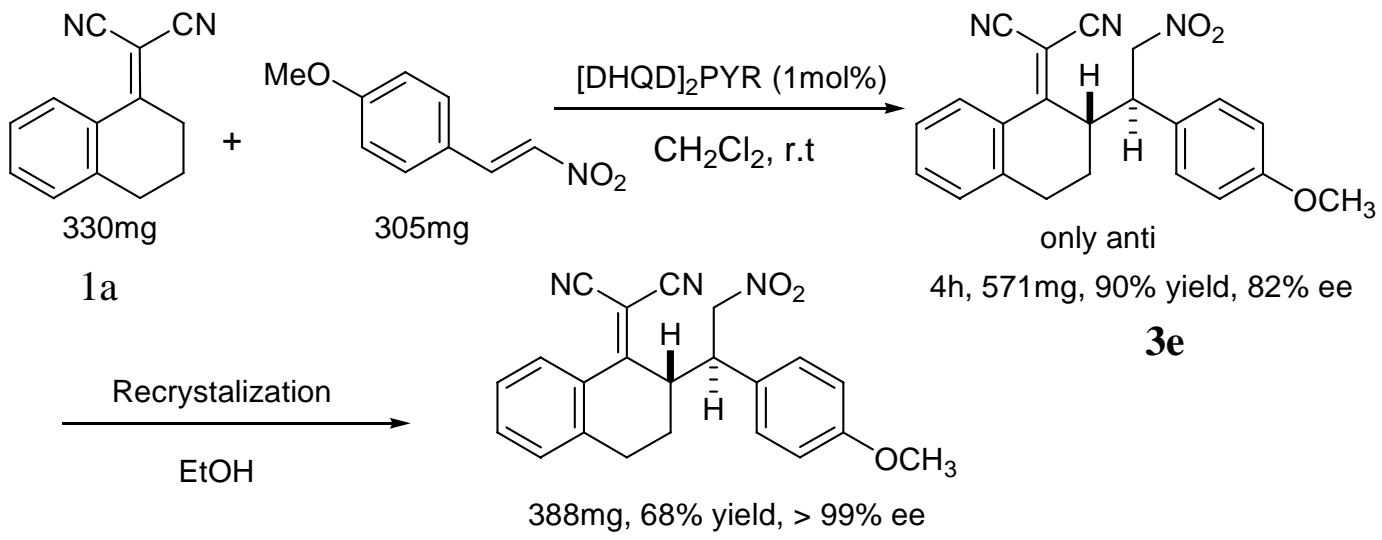

3e

[DHQD] ${ }_{2}$ PYR (15 mg, $0.017 \mathrm{mmol}$ ), activated alkene 1a (330 mg, 1.7,mmol) and p-MeO-nitrostyrene (305 mg, $1.7 \mathrm{mmol}$ ) were added to a flask in turn and then freshly distilled $\mathrm{CH}_{2} \mathrm{Cl}_{2}(15 \mathrm{~mL})$ was added. The solution was stirred at room temperature and monitored by TLC. After $4 \mathrm{~h}$, the reaction was completed. The solution was concentrated and flash chromatography (ethyl acetate/petroleum ether = 1: 10) gave the vinylogous Michael addition product 3e 571mg. Yield: 90\%, ee: 82\%. Enantiopure compound was obtained by recrystalization form EtOH (50mL). 
Yield: 68\%, ee: $99.6 \%$. mp: $174.9-175.2^{\circ} \mathrm{C} ;[\alpha]_{\mathrm{D}}{ }^{25}-201.5^{\circ}$ (c $\left.0.25, \mathrm{CHCl}_{3}\right)$.<smiles>COc1ccc(C(CN[N+](=O)[O-])C2CCc3ccccc3C2=C(C#N)C#N)cc1</smiles>

$3 e$

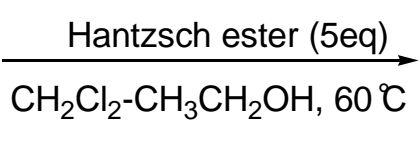<smiles>CCOC(=O)C1=C(C)NC(C)=C(C(=O)OCC)C1</smiles>

Hantzsch ester<smiles>COc1ccc(C(C[N+](=O)[O-])[C@H]2CCc3ccccc3[C@]2(C)C(C#N)C#N)cc1</smiles>

48h, $87 \%$ Yield $>99 \%$ ee

Hantzsch ester (339 mg, $1.33 \mathrm{mmol}$ ) was added to a stirred solution of vinylogous Michael addition product $3 \mathbf{e}(100 \mathrm{mg}, 0.26 \mathrm{mmol})$ in $\mathrm{DCM}-\mathrm{CH}_{3} \mathrm{CH}_{2} \mathrm{OH}(1: 1,10 \mathrm{~mL})$. The solution was stirred at $60{ }^{\circ} \mathrm{C}$ and monitored by TLC. After $48 \mathrm{~h}$, the reaction was completed. The solution was concentrated and flash chromatography (ethyl acetate/petroleum ether $=1$ : 11 ) gave the reductive product (Yield: 87\%). The cis-structure was determinated by NMR analysis (NOESY).

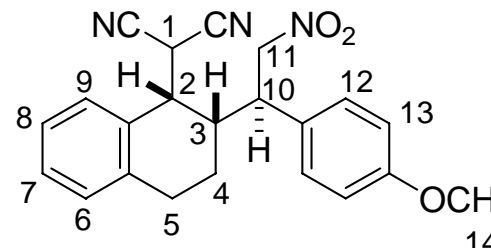
14 2-\{2-[1-(4-Methoxy-phenyl)-2-nitroethyl]-3,4-dihydro-naphthale n-1-yl\}-malononitrile (4). White Solid, m.p: $141-142{ }^{\circ} \mathrm{C} ;{ }^{1} \mathrm{H} \mathrm{NMR}\left(\mathrm{CDCl}_{3}, 600 \mathrm{MHz}\right)(\mathrm{H}-\mathrm{H}, \mathrm{C}-\mathrm{H}$ COSY, $\left.\mathrm{CDCl}_{3}, 300 \mathrm{MHz}\right) \delta(\mathrm{ppm}) 7.41$ (d, $\left.J=7.5 \mathrm{~Hz}, 1 \mathrm{H}\right), 7.34$ (t, $\left.J=7.5 \mathrm{~Hz}, 1 \mathrm{H}\right), 7.28$ (m, 1H), 7.20 (d, $J=7.5 \mathrm{~Hz}, 1 \mathrm{H}$ ), 7.14 (d, $J=8.6 \mathrm{~Hz}, 2 \mathrm{H}, H-12$ ), 6.93 (d, $J=8.6 \mathrm{~Hz}, 2 \mathrm{H}, H-13$ ), 4.83-4.80 (m, 1H, H-11), 4.69-4.66 (m, 1H, H-11), 4.07 (d, $J=2.6 \mathrm{~Hz}, 1 \mathrm{H}, H-1$ ), 3.83 (s, 3H, H-14), 3.56-3.53 (m, 2H), 3.04 (dd, $J=18.1,7.3$ Hz, 1H, H-5), 2.84-78 (m, 1H, H-5), 2.41-2.37 (m, 1H, $H-3), 1.82-1.78(\mathrm{~m}, 1 \mathrm{H}, H-4), 1.72-1.69$ (m, $1 \mathrm{H}, H-4) ;{ }^{13} \mathrm{C} \mathrm{NMR}\left(\mathrm{CDCl}_{3}, 150 \mathrm{MHz}\right) \delta(\mathrm{ppm})$ 159.7, 136.2, 132.7, 129.9, 129.4, 129.2, 128.9, 128.7, 126.5, 114.9, 113.0, 111.5, 79.0, 55.3, 46.2, 43.4, 40.9, 27.7, 24.8, 21.1; IR (KBr): v 3449, 2912, 2255, 1610, 1553, 1513, 1381, 1255, 1181, 1031, 831, $739 \mathrm{~cm}^{-1}$; HRMS (ESI) calcd for $\mathrm{C}_{22} \mathrm{H}_{21} \mathrm{~N}_{3} \mathrm{O}_{3}+\mathrm{Na}$ : 398.1475, found: 398.1482; >99.5\% ee was determined by HPLC on AS column, 30\% 2-propanol/hexane, 1.0ml/min, UV273nm, $\mathrm{t}_{\text {major }}$ $=20.7 \mathrm{~min} ;[\alpha]_{\mathrm{D}}{ }^{25}-152.6^{\circ}(c \mathrm{c} 0.17, \mathrm{THF})$. 
<smiles>COc1ccc([C@H](C[N+](=O)[O-])[C@H]2CCc3ccccc3C2=C(C#N)C#N)cc1</smiles>

$3 e$

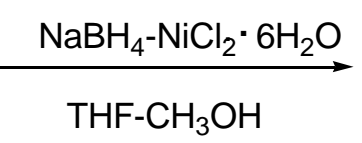

THF- $\mathrm{CH}_{3} \mathrm{OH}$

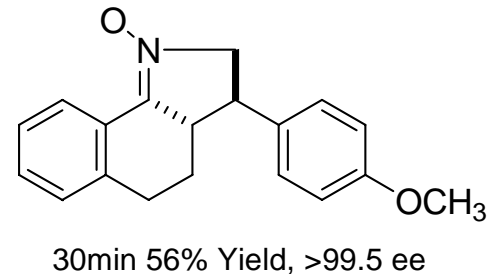

5

$\mathrm{NaBH}_{4}$ (21 mg, $0.054 \mathrm{mmol}$ ) was added to a stirred solution of enantiopure vinylogous Michael addition product $3 \mathbf{e}(200 \mathrm{mg}, 0.54 \mathrm{mmol})$ and $\mathrm{NiCl}_{2} \cdot 6 \mathrm{H}_{2} \mathrm{O}(128 \mathrm{mg}, 0.54 \mathrm{mmol})$ in THF- $\mathrm{CH}_{3} \mathrm{OH}$ (1:1, $5 \mathrm{~mL}$ ). The mixture was stirred at room temperature and monitored by TLC. After 30 min, the reaction was completed. The solution was removed under reduced pressure and flash chromatography (ethyl acetate/petroleum ether $=1: 10-2: 1$ ) gave the product (Yield: $56 \%$, Ee $>99.5 \%)$.<smiles>COc1ccc(C2CN(O)C3c4ccccc4CCC23)cc1</smiles>

3-(4-Methoxy-phenyl)-3,3a,4,5-terahydro-2H-benzo[g]indol-1-ol. (5) White Solid, mp: $120^{\circ} \mathrm{C}$ (decomposed); ${ }^{1} \mathrm{H}$ NMR $\left(\mathrm{CDCl}_{3}, 600 \mathrm{MHz}\right) \delta$ (ppm) 9.37-9.36 (m, 1H), 7.37-7.33 (m, 1H), 7.27 (d, $J=8.3 \mathrm{~Hz}, 2 \mathrm{H}), 7.23-7.22(\mathrm{~m}, 1 \mathrm{H}), 6.94$ (d, $J=8.5 \mathrm{~Hz}, 2 \mathrm{H})$, 4.44-4.39 (m, 1H), 4.33-4.29 (m, 1H), 3.83 (s, 3H), 3.37-3.32 (m, 1H), 3.22 (bs, 1H), 2.96-2.86 (m, 2H), 2.19-2.05 (m, 1H), 1.72-1.65 (m, 1H); ${ }^{13} \mathrm{C} \mathrm{NMR}\left(\mathrm{CDCl}_{3}, 150 \mathrm{MHz}\right) \delta(\mathrm{ppm})$ 159.1, 140.9, 138.5, 130.5, 128.5, 128.50, 127.2, 126.7, 125.8, 114.5, 114.2, 69.9, 55.4, 50.3, 45.5, 30.2, 27.7; IR (KBr): v 3458, 2934, 2835, 1609, 1569, 1512, 1469, 1442, 1382, 1301, 1246, 1218, 1179, 1122, 1032, 824, 794, 755, 734, 740, 612, 580 $\mathrm{cm}^{-1}$; HRMS (ESI) calcd for $\mathrm{C}_{19} \mathrm{H}_{19} \mathrm{NO}_{2}+\mathrm{Na}$ : 316.1306, found: 316.1316; >99.5\% ee was determined by HPLC on AS column, 30\% 2-propanol/hexane, $1.0 \mathrm{ml} / \mathrm{min}$, UV 254nm, $\mathrm{t}_{\text {major }}=22.4 \mathrm{~min}, \mathrm{t}_{\text {minor }}=48.9 ;[\alpha]_{\mathrm{D}}{ }^{25}-14.3^{\circ}$ (c 0.24, EtOAc). The structure of 5 was further proved by X-ray crystallographic analysis of racemic 5 (Figure 4).

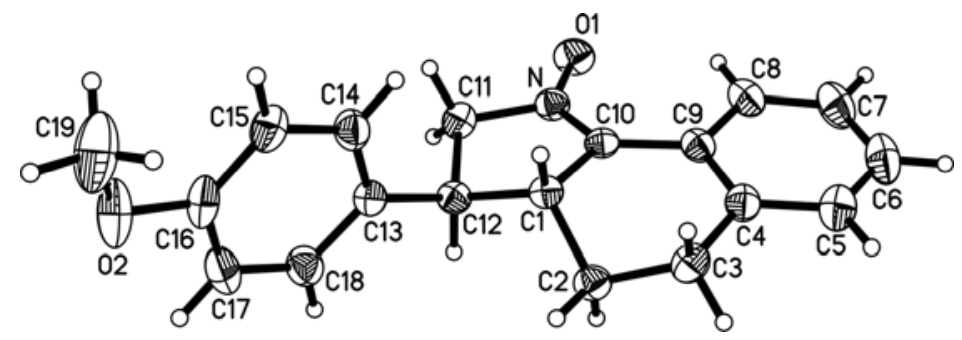

Figure 4 X-Ray crystallographic structure of rac-5. 
6. Crystal data of compounds, 2, rac-3e, 3c, and rac-5

1) Crystal data of 2-Benzyl-2-(3,4-dihydro-naphthalen-1-yl)-malononitrile, 2

\begin{tabular}{|c|c|}
\hline Identification code & y1893 \\
\hline Empirical formula & $\mathrm{C}_{20} \mathrm{H}_{16} \mathrm{~N}_{2}$ \\
\hline Formula weight & 284.35 \\
\hline Temperature & $287(2) \mathrm{K}$ \\
\hline Wavelength & $0.71073 \mathrm{~A}$ \\
\hline Crystal system & Triclinic \\
\hline Space group & $\mathrm{P}-1$ \\
\hline \multirow[t]{3}{*}{ Unit cell dimensions } & $\mathrm{a}=8.829(1) \mathrm{A} \quad$ alpha $=78.48(1) \mathrm{deg}$. \\
\hline & beta $=88.69(1) \mathrm{deg}$. \\
\hline & $\mathrm{c}=20.210(2) \mathrm{A} \quad$ gamma $=84.74(1) \mathrm{deg}$ \\
\hline Volume, Z & 1566.7(3) A^3, 4 \\
\hline Density (calculated) & $1.205 \mathrm{Mg} / \mathrm{m}^{\wedge} 3$ \\
\hline Absorption coefficient & $0.071 \mathrm{~mm}^{\wedge}-1$ \\
\hline $\mathrm{F}(000)$ & 600 \\
\hline Crystal size & $0.56 \times 0.34 \times 0.30 \mathrm{~mm}$ \\
\hline Theta range for data collection & 1.03 to $25.50 \mathrm{deg}$. \\
\hline Limiting indices & $0<=\mathrm{h}<=10,-10<=\mathrm{k}<=10,-24<=\mathrm{l}<=24$ \\
\hline Reflections collected & 6417 \\
\hline Independent reflections & $5804[\mathrm{R}(\mathrm{int})=0.0135]$ \\
\hline Absorption correction & None \\
\hline Refinement method & Full-matrix least-squares on $\mathrm{F}^{\wedge} 2$ \\
\hline Data / restraints / parameters & 5804 / 0 / 398 \\
\hline Goodness-of-fit on $\mathrm{F}^{\wedge} 2$ & 0.870 \\
\hline Final R indices [I $>2 \operatorname{sigma}(\mathrm{I})]$ & $\mathrm{R} 1=0.0594, \mathrm{wR} 2=0.1624$ \\
\hline $\mathrm{R}$ indices (all data) & $\mathrm{R} 1=0.1113, \mathrm{wR} 2=0.1836$ \\
\hline Extinction coefficient & $0.0053(18)$ \\
\hline Largest diff. peak and hole & 0.507 and -0.260 e. $\mathrm{A}^{\wedge}-3$ \\
\hline
\end{tabular}

2) Crystal data of 2-\{2-[1-(4-Methoxy-phenyl)-2-nitroethyl]-3,4-dihydro-2H-naphthalenylidene\}-malononitrile, rac-3e

$\begin{array}{lc}\text { Identification code } & \mathrm{y} 1892 \\ \text { Empirical formula } & \mathrm{C}_{22} \mathrm{H}_{19} \mathrm{~N}_{3} \mathrm{O}_{3} \\ \text { Formula weight } & 373.40 \\ \text { Temperature } & 287(2) \mathrm{K} \\ \text { Wavelength } & 0.71073 \mathrm{~A} \\ \text { Crystal system } & \text { Monoclinic }\end{array}$


Space group

Unit cell dimensions

Volume, Z

Density (calculated)

Absorption coefficient

$\mathrm{F}(000)$

Crystal size

Theta range for data collection

Limiting indices

Reflections collected

Independent reflections

Absorption correction

Refinement method

Data / restraints / parameters

Goodness-of-fit on $\mathrm{F}^{\wedge} 2$

Final R indices [I $>2 \operatorname{sigma}(\mathrm{I})]$

$\mathrm{R}$ indices (all data)

Extinction coefficient

Largest diff. peak and hole
$\mathrm{P} 2(1) / \mathrm{c}$

$$
\begin{array}{ll}
\mathrm{a}=7.074(1) \mathrm{A} & \text { alpha }=90 \mathrm{deg} . \\
\mathrm{b}=15.601(2) \mathrm{A} & \text { beta }=91.76(2) \mathrm{deg} . \\
\mathrm{c}=17.909(3) \mathrm{A} & \text { gamma }=90 \mathrm{deg} .
\end{array}
$$

$$
\text { 1975.51(55) A^3, } 4
$$

$1.255 \mathrm{Mg} / \mathrm{m}^{\wedge} 3$

$0.085 \mathrm{~mm}^{\wedge}-1$

784

$0.52 \times 0.40 \times 0.32 \mathrm{~mm}$

1.73 to $25.50 \mathrm{deg}$.

$0<=\mathrm{h}<=8,0<=\mathrm{k}<=18,-21<=\mathrm{l}<=21$

4293

3679 [R(int) $=0.0161]$

None

Full-matrix least-squares on $\mathrm{F}^{\wedge} 2$

3679 / 0 / 255

0.878

$\mathrm{R} 1=0.0416, \mathrm{wR} 2=0.0928$

$\mathrm{R} 1=0.0858, \mathrm{wR} 2=0.1048$

0.0193(13)

0.118 and -0.135 e. $\mathrm{A}^{\wedge}-3$

\section{3) Crystal data of 2-\{2-[1-(4-Chloro-phenyl)-2-nitroethyl]-3,4-dihydro-2H-naphthalen-1-}

\section{ylidene\}-malononitriole, $3 c$}

Identification code

Empirical formula

Formula weight

Temperature

Wavelength

Crystal system

Space group

Unit cell dimensions

Volume, Z

Density (calculated)

Absorption coefficient

$\mathrm{F}(000)$

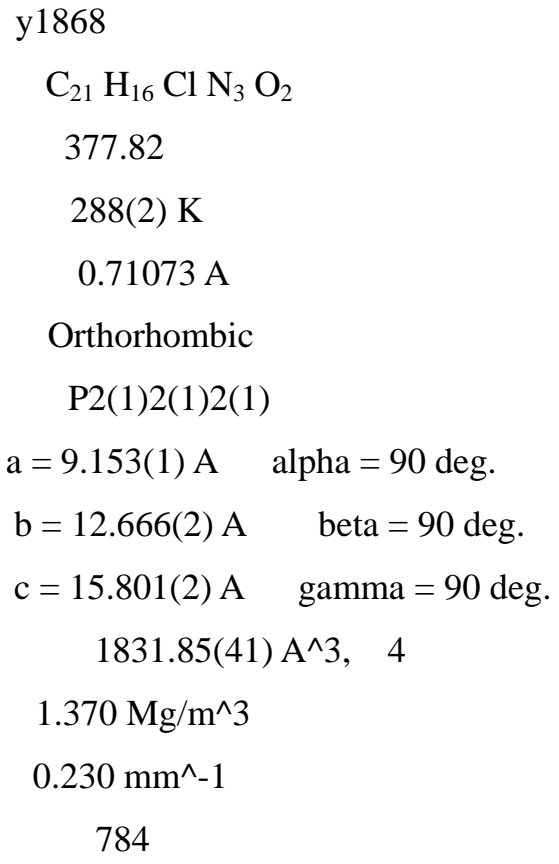


Theta range for data collection $\quad 2.06$ to $27.00 \mathrm{deg}$.

Limiting indices

Reflections collected

Independent reflections

Absorption correction

Max. and min. transmission

Refinement method

Data / restraints / parameters

Goodness-of-fit on $\mathrm{F}^{\wedge} 2$

Final R indices [I $>2 \operatorname{sigma}(\mathrm{I})]$

$\mathrm{R}$ indices (all data)

Absolute structure parameter

Extinction coefficient

Largest diff. peak and hole $-11<=\mathrm{h}<=11,-16<=\mathrm{k}<=16,-20<=\mathrm{l}<=20$

4770

$4000[\mathrm{R}(\mathrm{int})=0.0172]$

Empirical

0.9785 and 0.8775

Full-matrix least-squares on $\mathrm{F}^{\wedge} 2$

4000 / 0 / 245

0.966

$\mathrm{R} 1=0.0460, \mathrm{wR} 2=0.1045$

$\mathrm{R} 1=0.0716, \mathrm{wR} 2=0.1138$

$0.14(9)$

$0.0122(16)$

0.351 and -0.251 e. $\mathrm{A}^{\wedge}-3$

\section{4) Crystal data of 3-(4-Methoxy-phenyl)-3,3a,4,5-tetrahydro-2H-benzo[g]indol-1-ol, rac-5}

Identification code

Empirical formula

Formula weight

Temperature

Wavelength

Crystal system

Space group

Unit cell dimensions

Volume, Z

Density (calculated)

Absorption coefficient

$\mathrm{F}(000)$

Crystal size

Theta range for data collection

Limiting indices

Reflections collected

Independent reflections

Absorption correction

Refinement method y1939

$\mathrm{C}_{19} \mathrm{H}_{19} \mathrm{~N} \mathrm{O}_{2}$

293.35

300(2) K

$0.71073 \mathrm{~A}$

Orthorhombic

Pbca

$\mathrm{a}=10.162(2) \mathrm{A} \quad$ alpha $=90 \mathrm{deg}$.

$\mathrm{b}=11.205(2) \mathrm{A} \quad$ beta $=90 \mathrm{deg}$.

$\mathrm{c}=27.271(5) \mathrm{A} \quad$ gamma $=90 \mathrm{deg}$.

3105.4(10) A^3, 8

$1.255 \mathrm{Mg} / \mathrm{m}^{\wedge} 3$

$0.081 \mathrm{~mm}^{\wedge}-1$

1248

$0.42 \times 0.36 \times 0.30 \mathrm{~mm}$

1.49 to $25.50 \mathrm{deg}$.

$0<=\mathrm{h}<=12,0<=\mathrm{k}<=13,-1<=\mathrm{l}<=33$

3497

2895 [R(int) $=0.0088]$

None

Full-matrix least-squares on $\mathrm{F}^{\wedge} 2$ 
Data / restraints / parameters

Goodness-of-fit on $\mathrm{F}^{\wedge} 2$

Final R indices [I $>2$ sigma(I)]

$\mathrm{R}$ indices (all data)

Extinction coefficient

Largest diff. peak and hole
2895 / 0 / 201

0.847

$\mathrm{R} 1=0.0412, \mathrm{wR} 2=0.0766$

$\mathrm{R} 1=0.1027, \mathrm{wR} 2=0.0896$

0.0034(3)

0.132 and -0.135 e. $\mathrm{A}^{\wedge}-3$

7. ${ }^{1} \mathrm{H},{ }^{13} \mathrm{C}$ NMR and HPLC charts of compounds, $2,3,4$, and 5

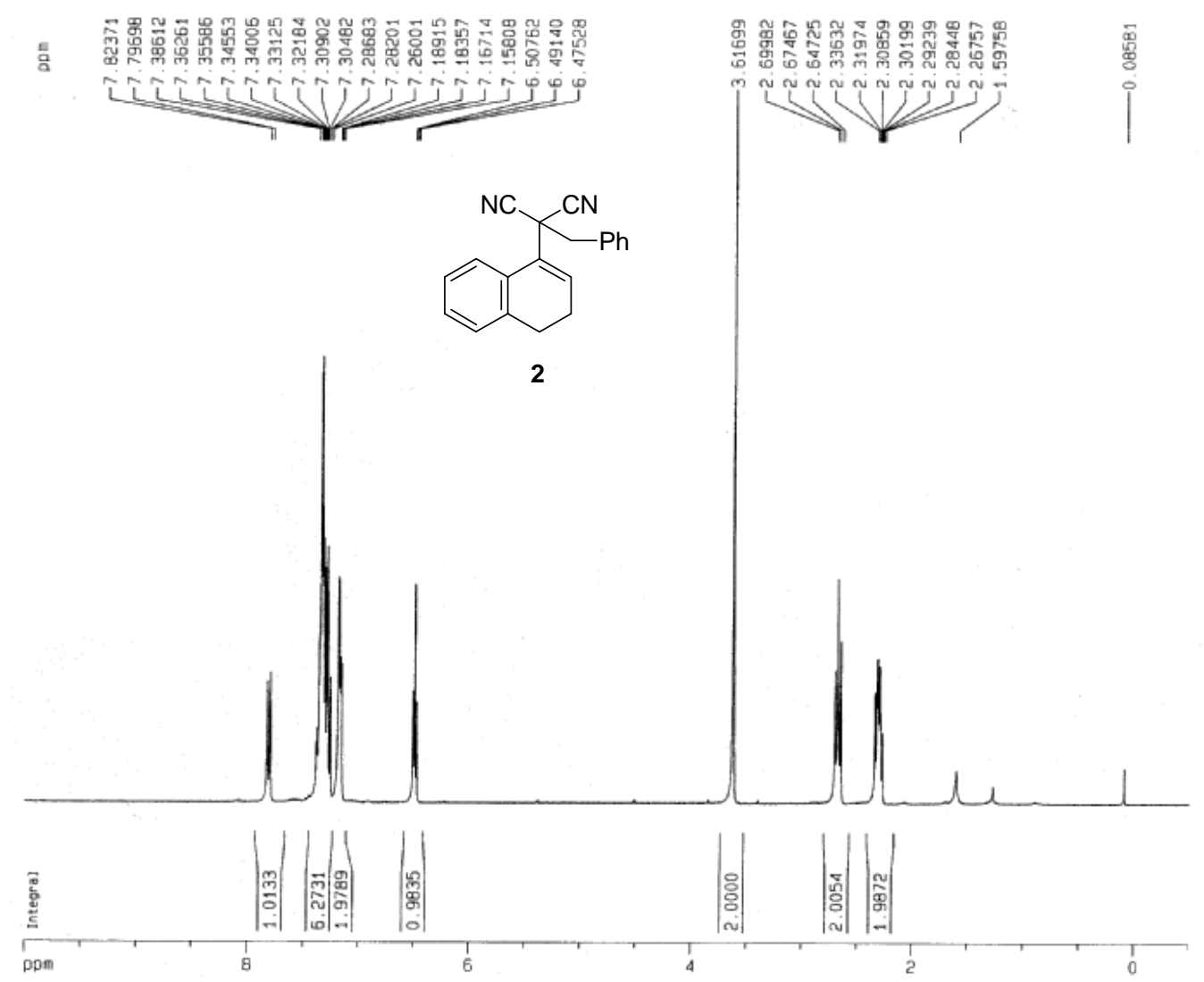



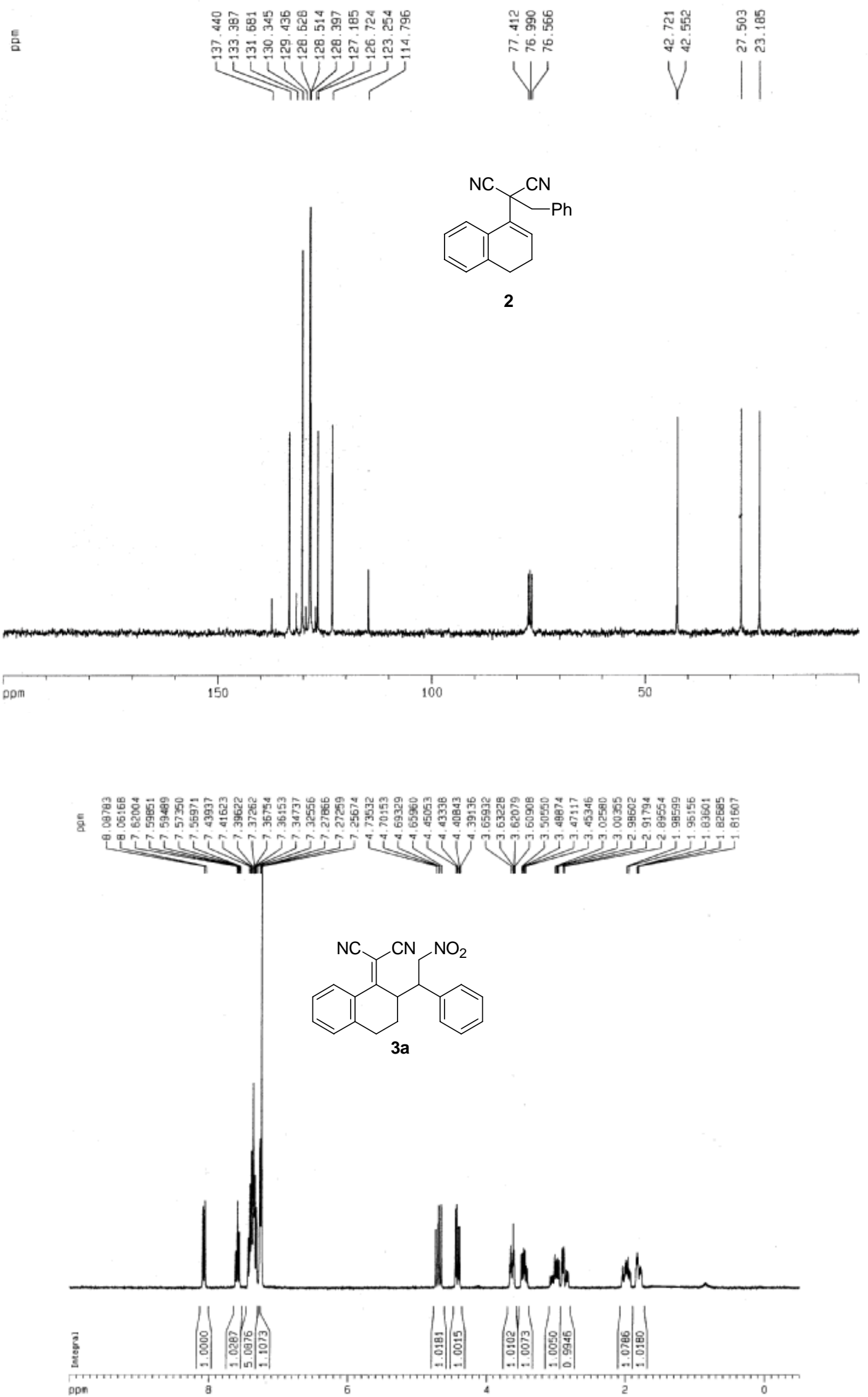

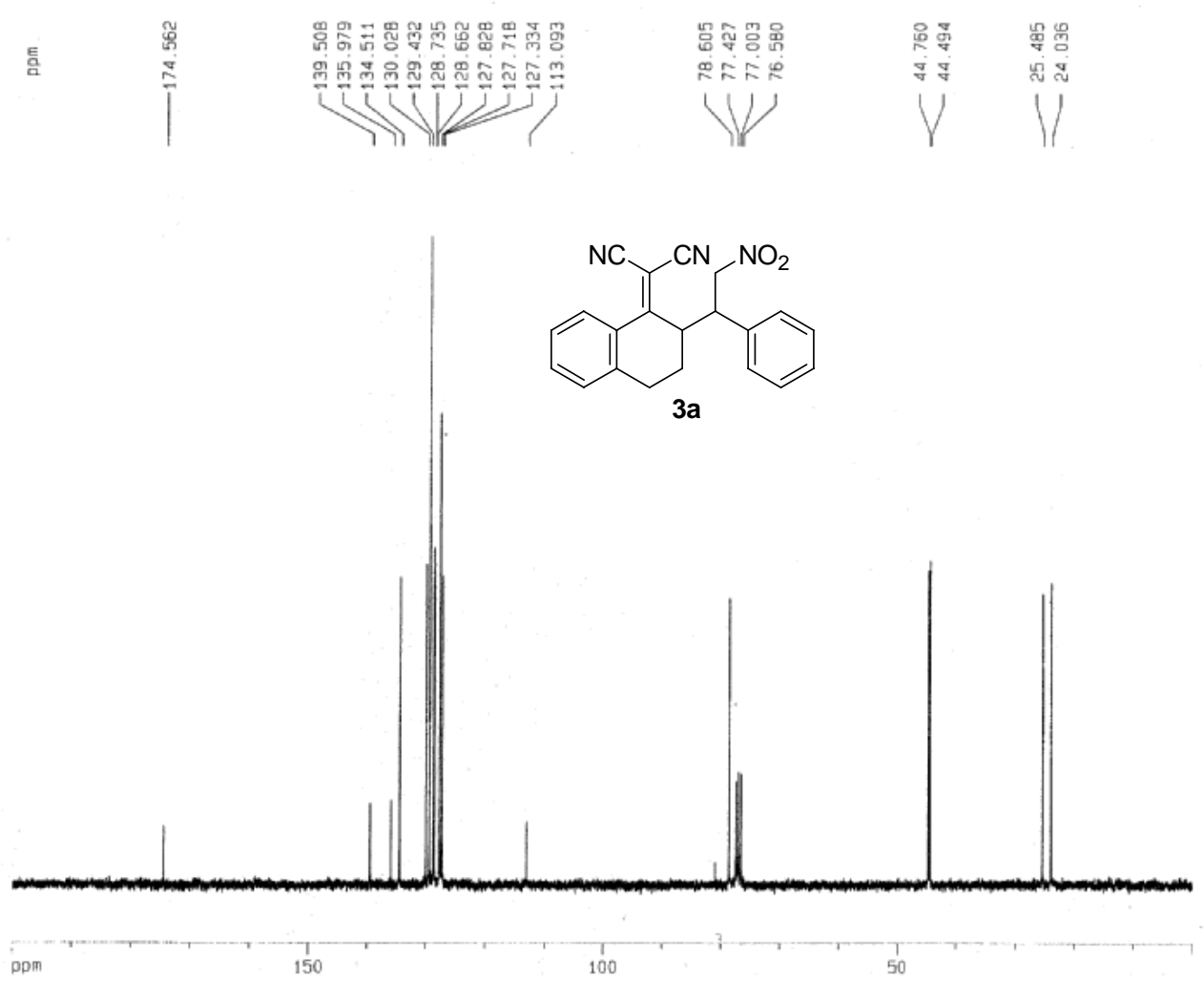

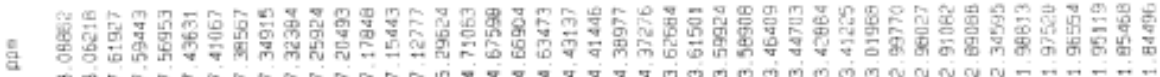

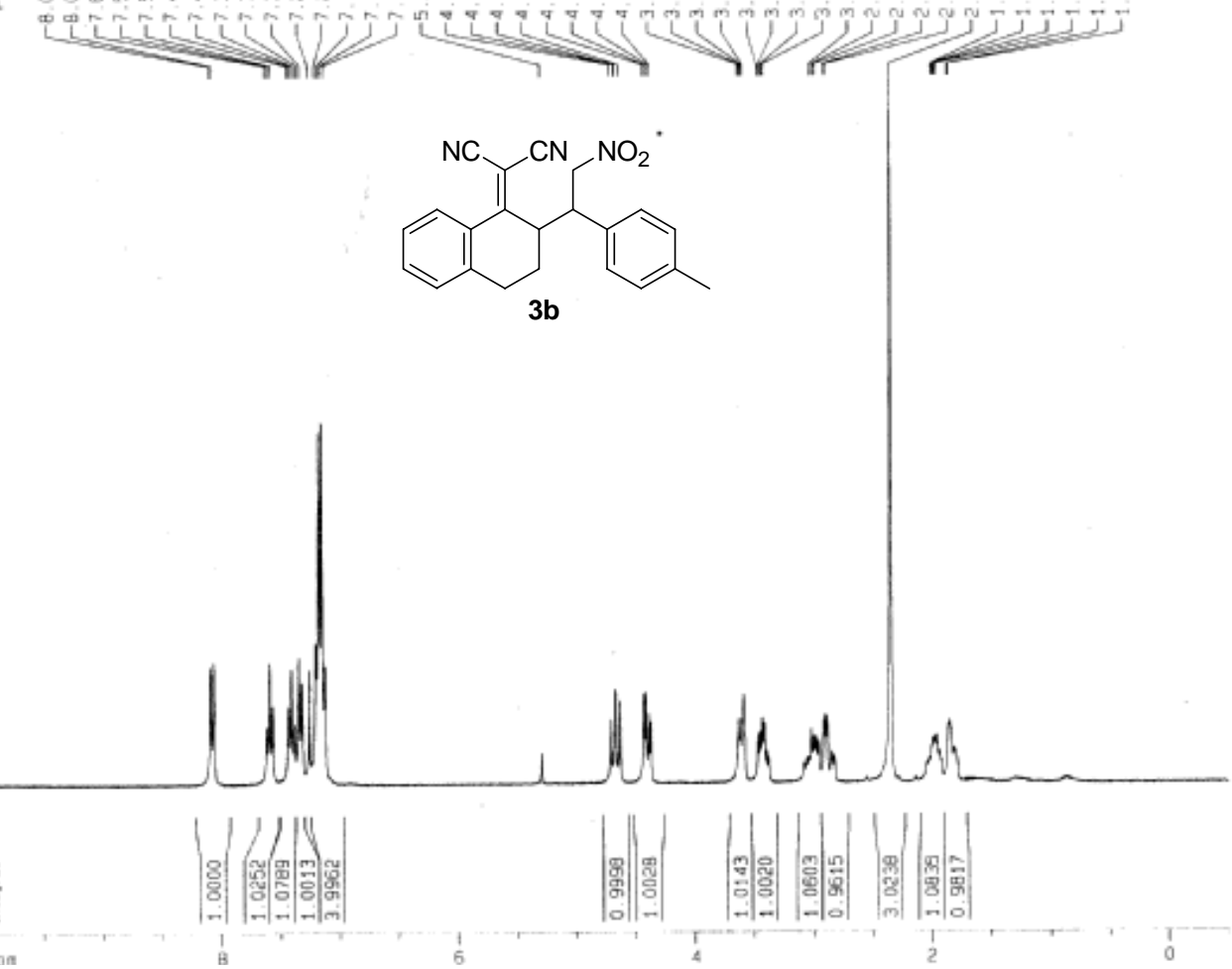




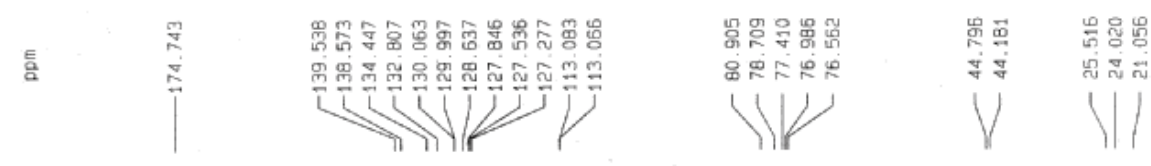

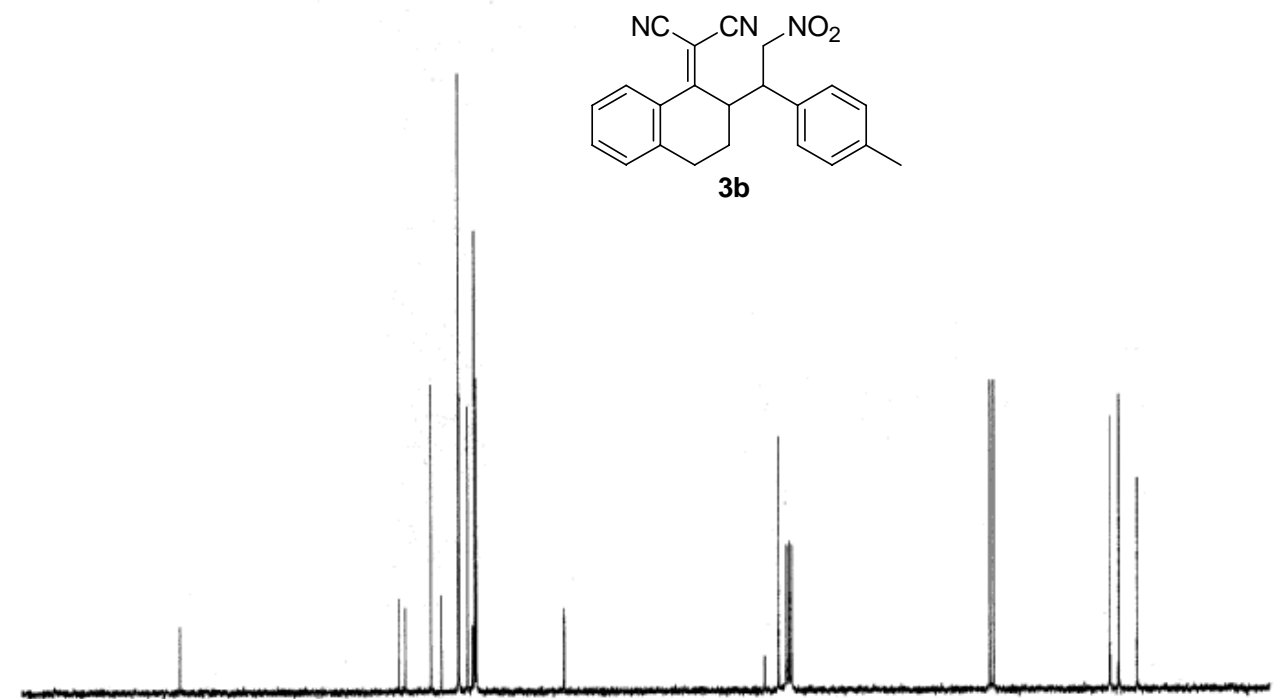

pom

$\frac{1}{150}$

100

50

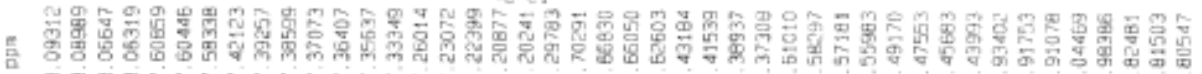

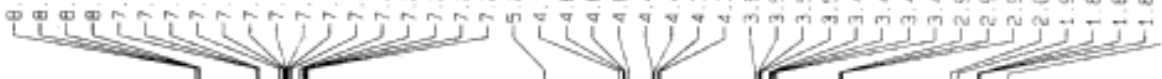

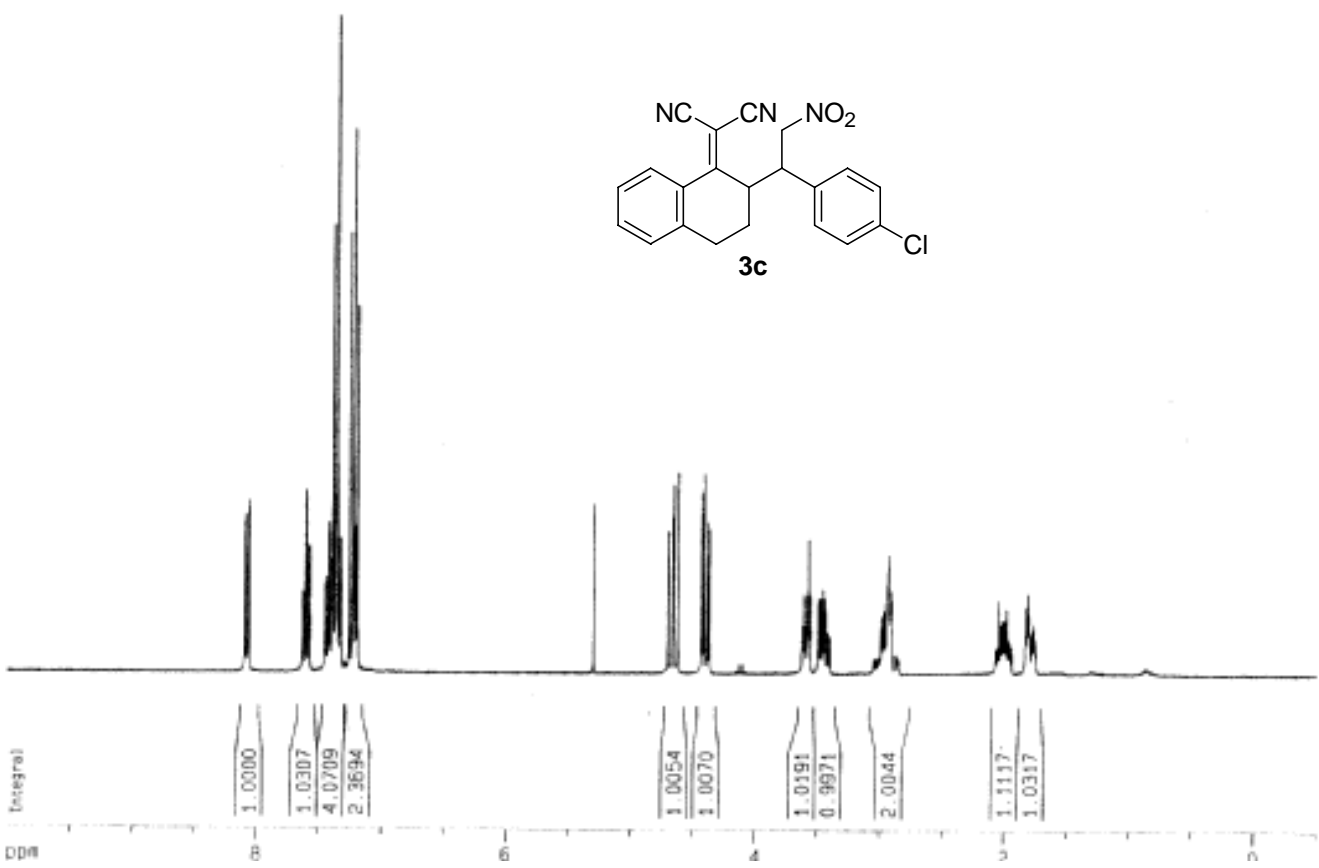




要言

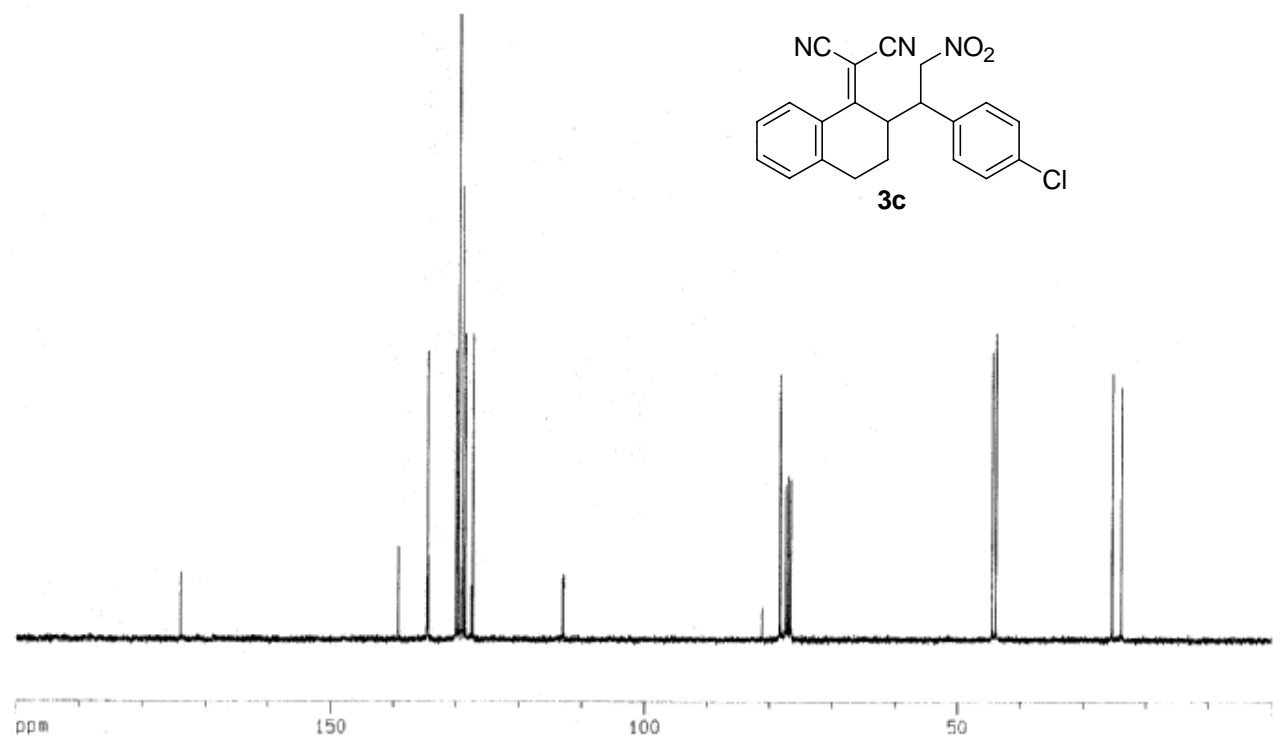

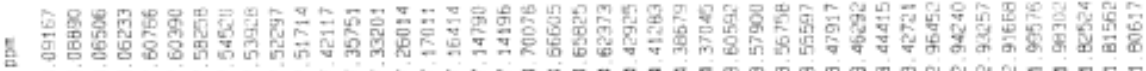
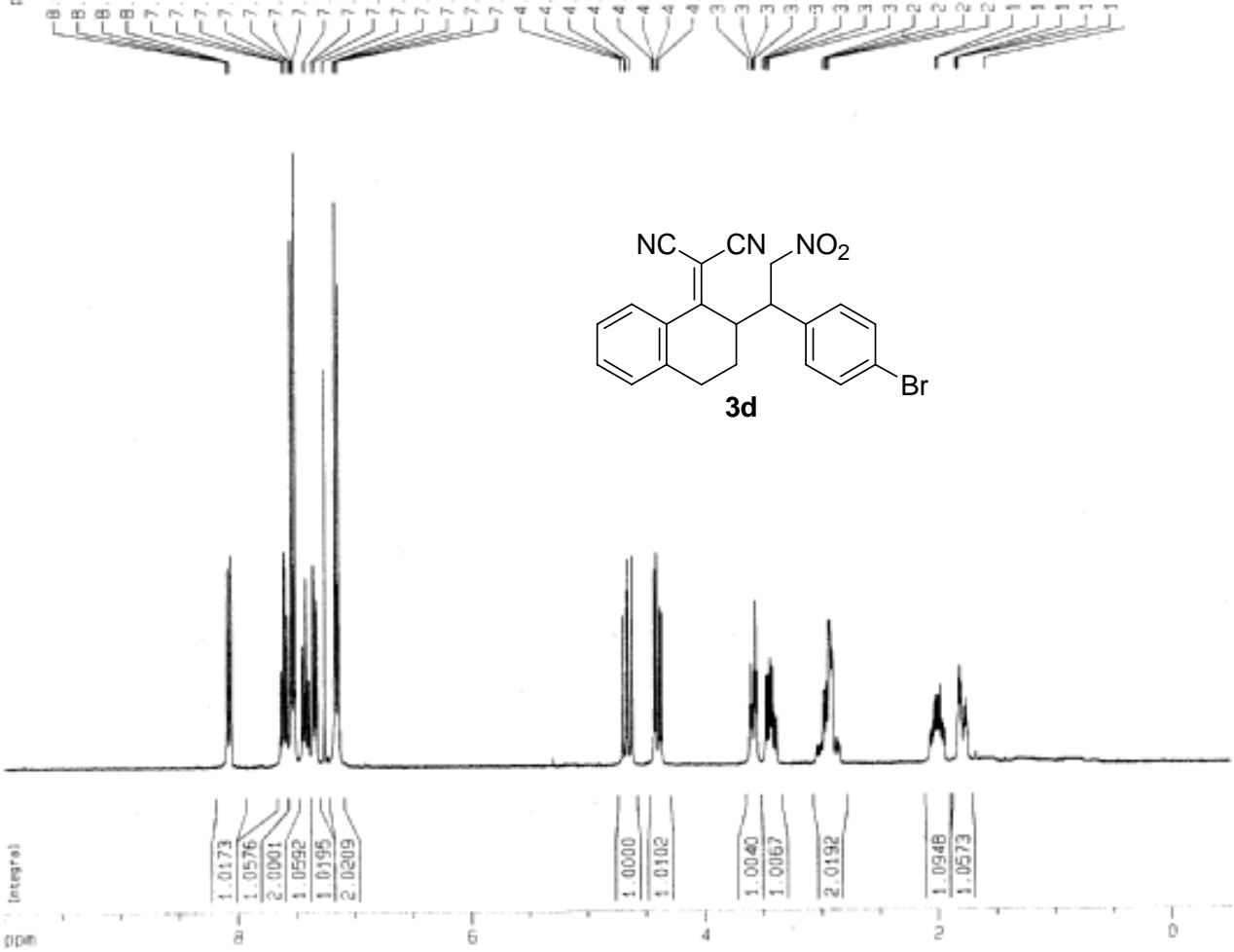

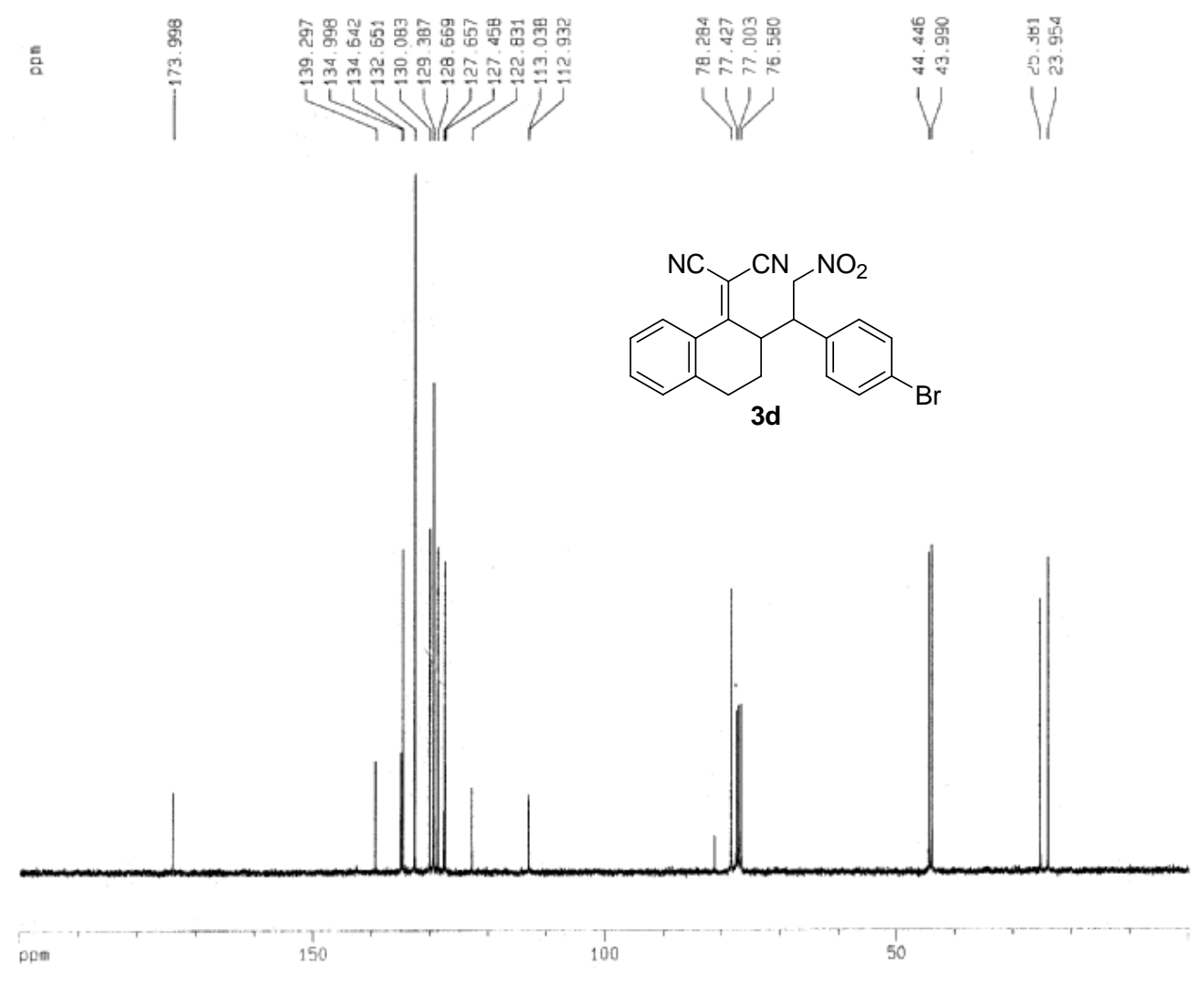

홍
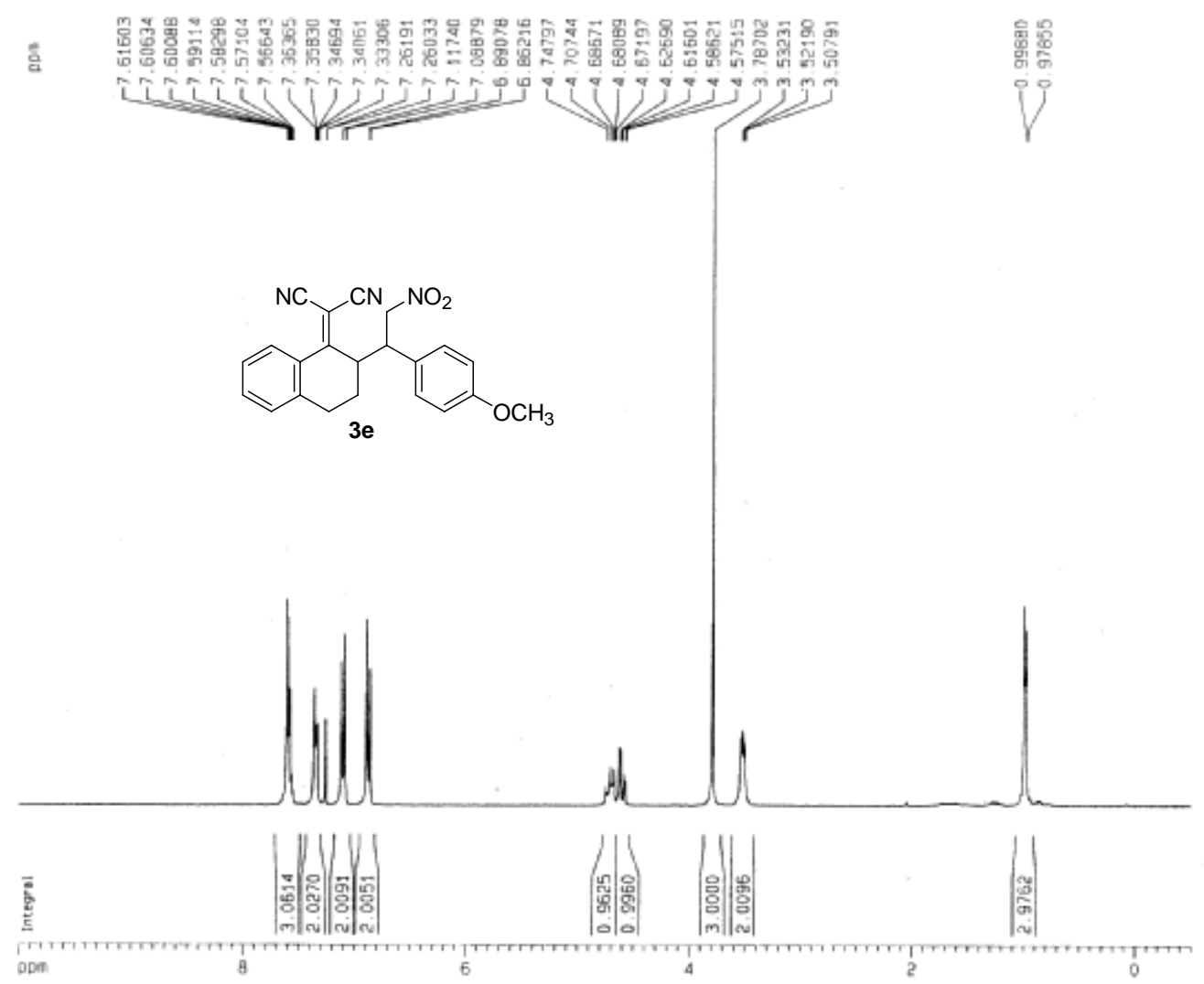

S21 


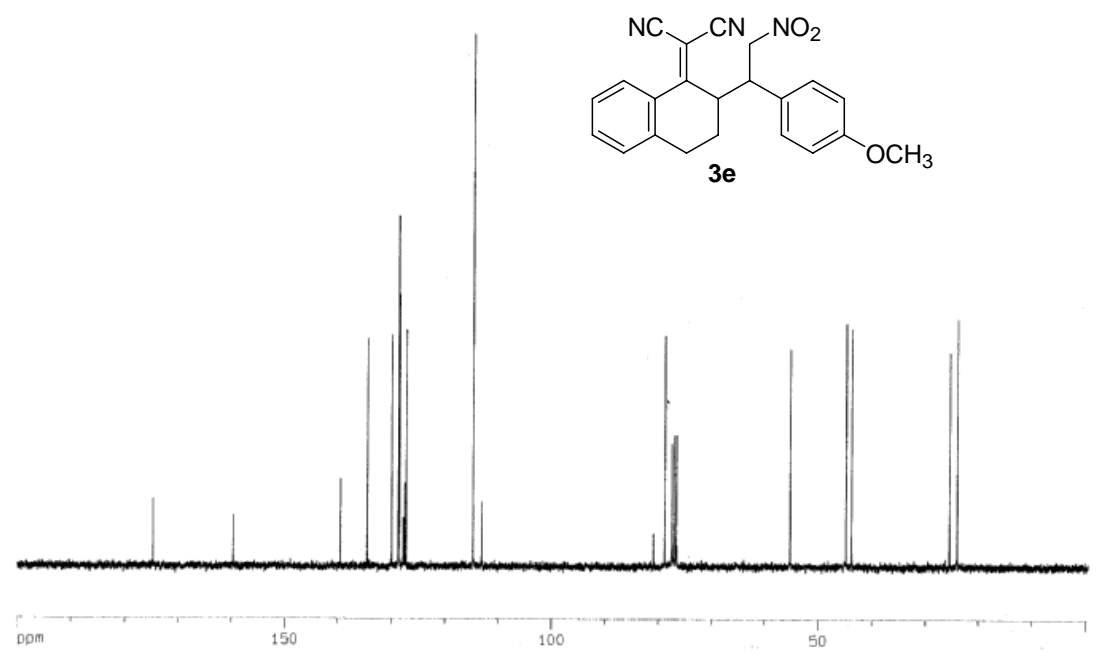

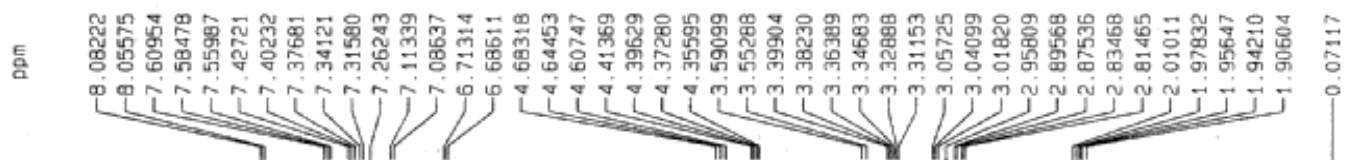<smiles>CC(C#N)=C1c2ccccc2CCC1C(C[Si])c1ccc(N(C)C)cc1</smiles>

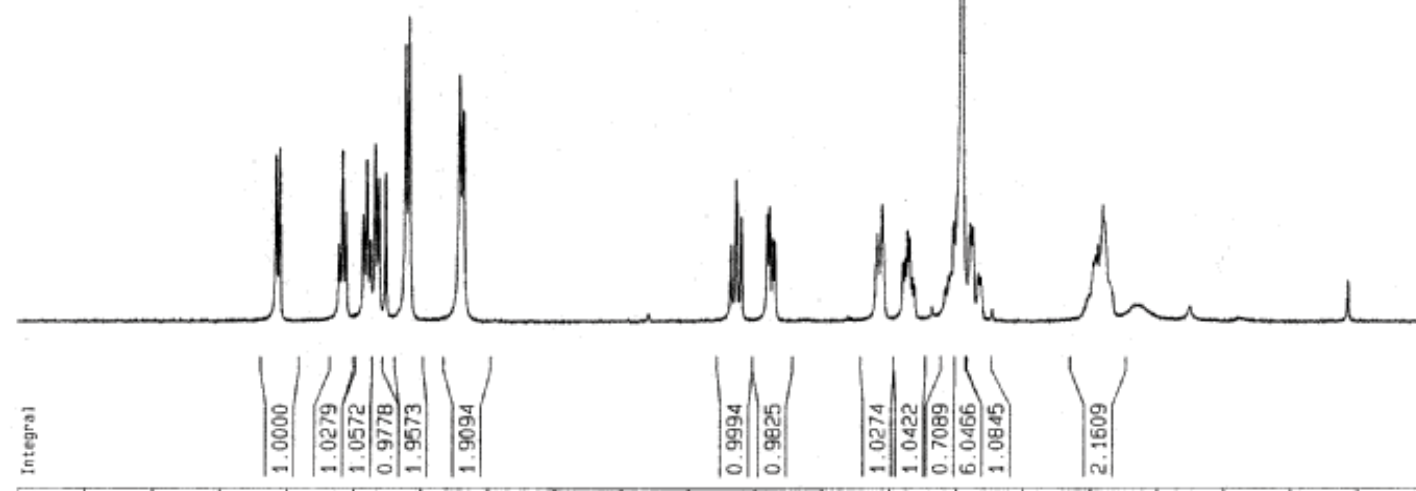



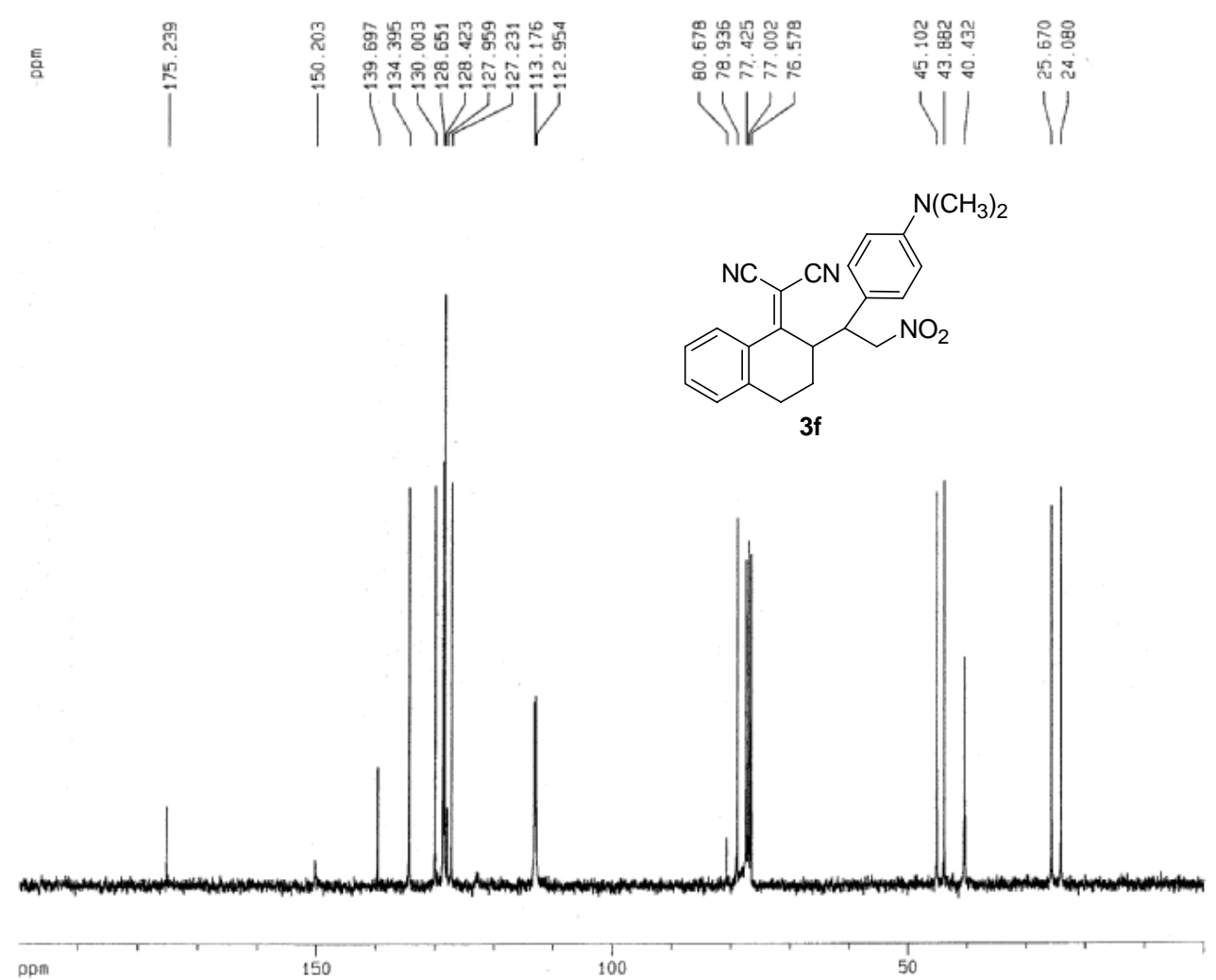

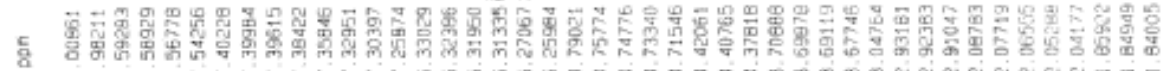

on

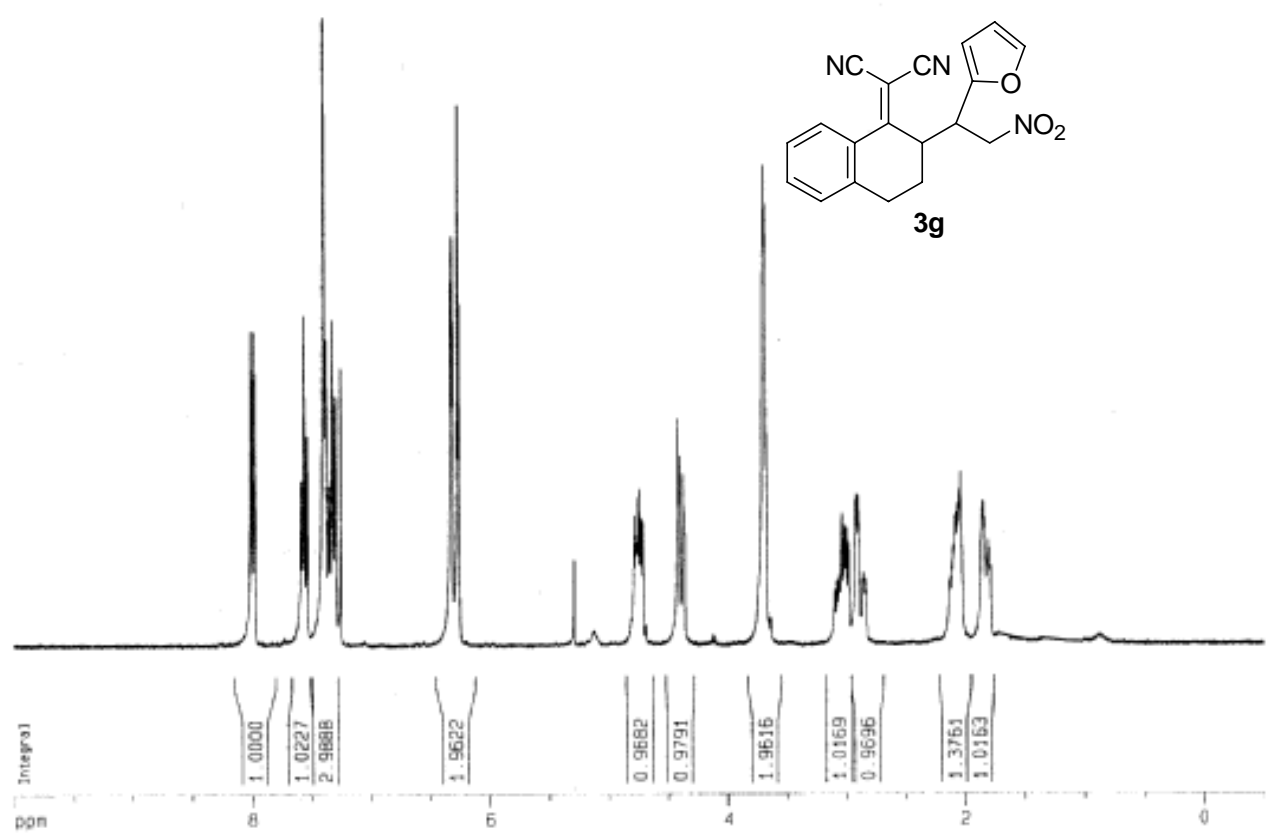




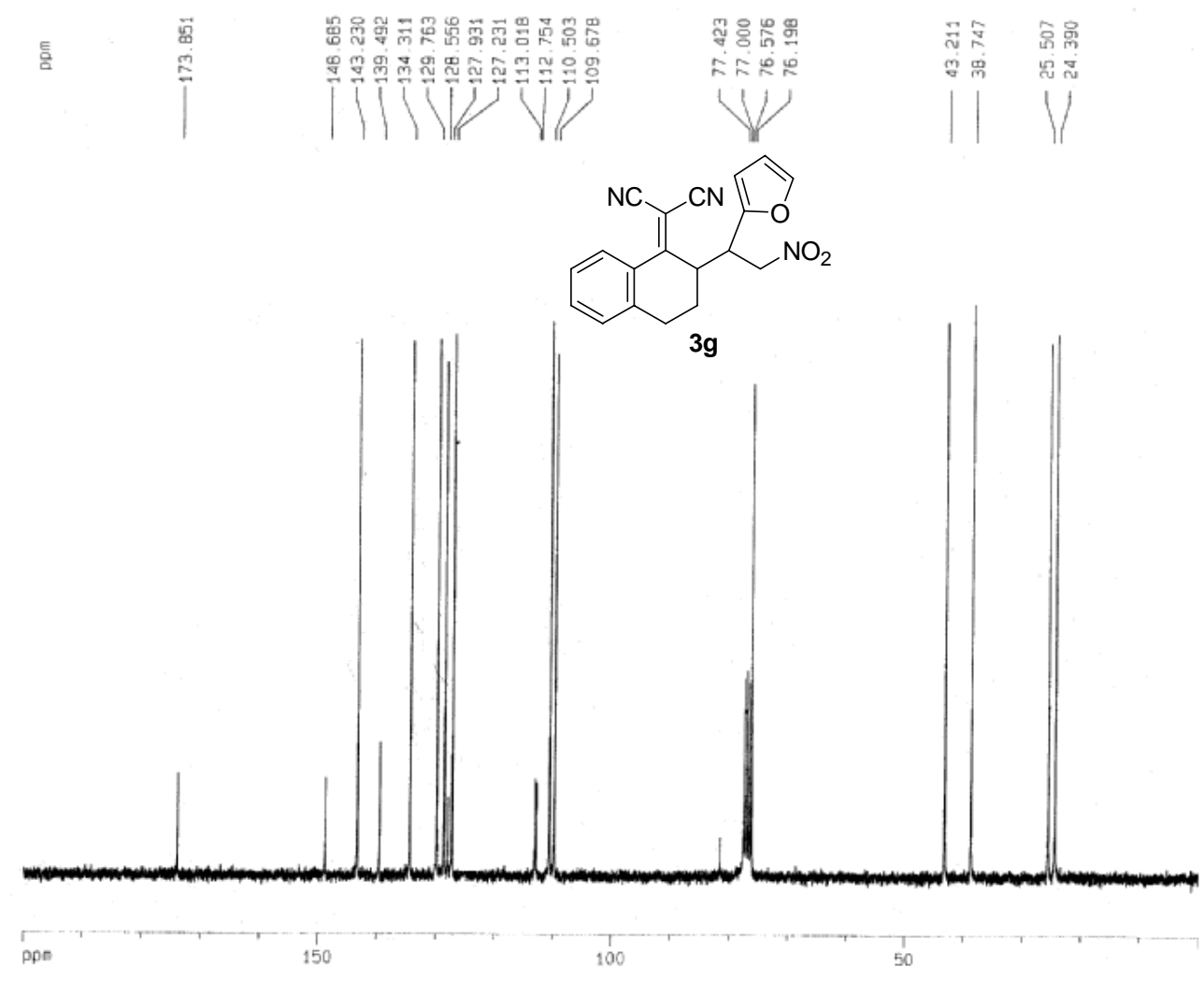

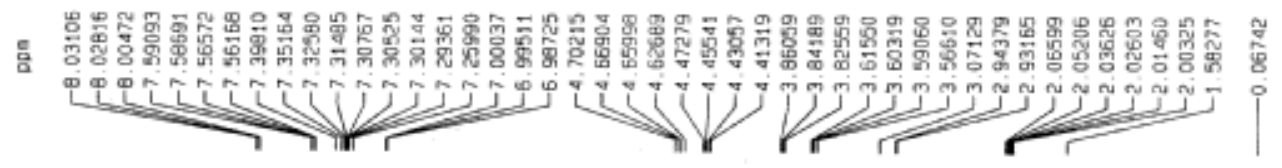

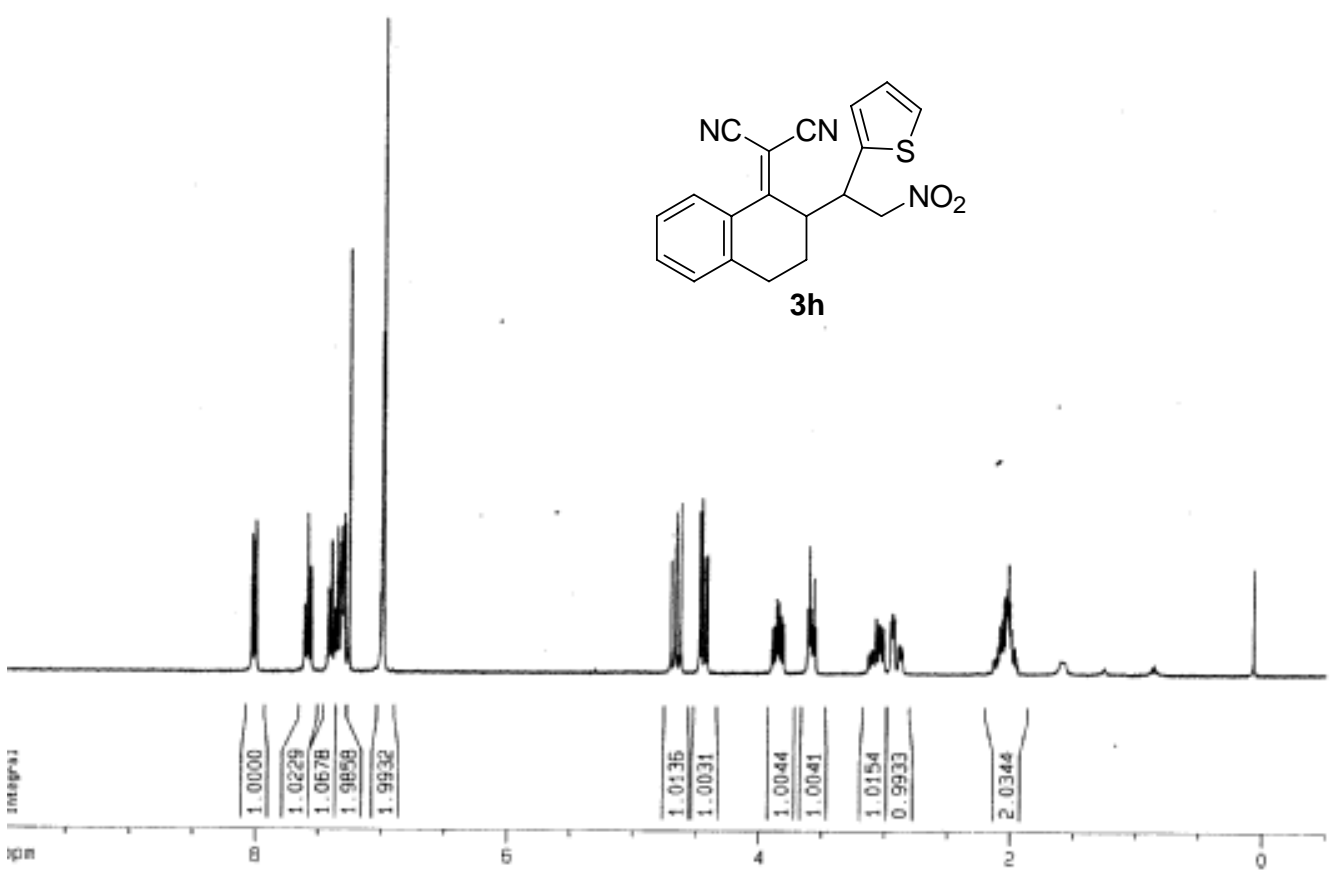




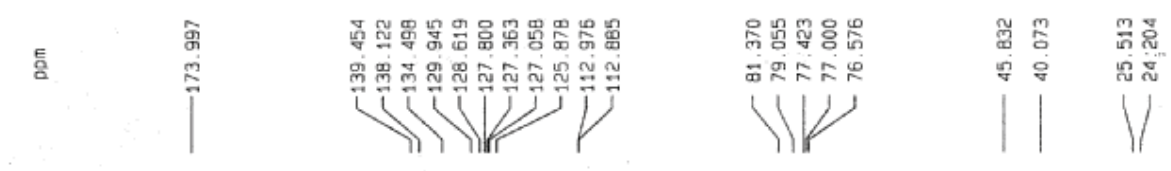

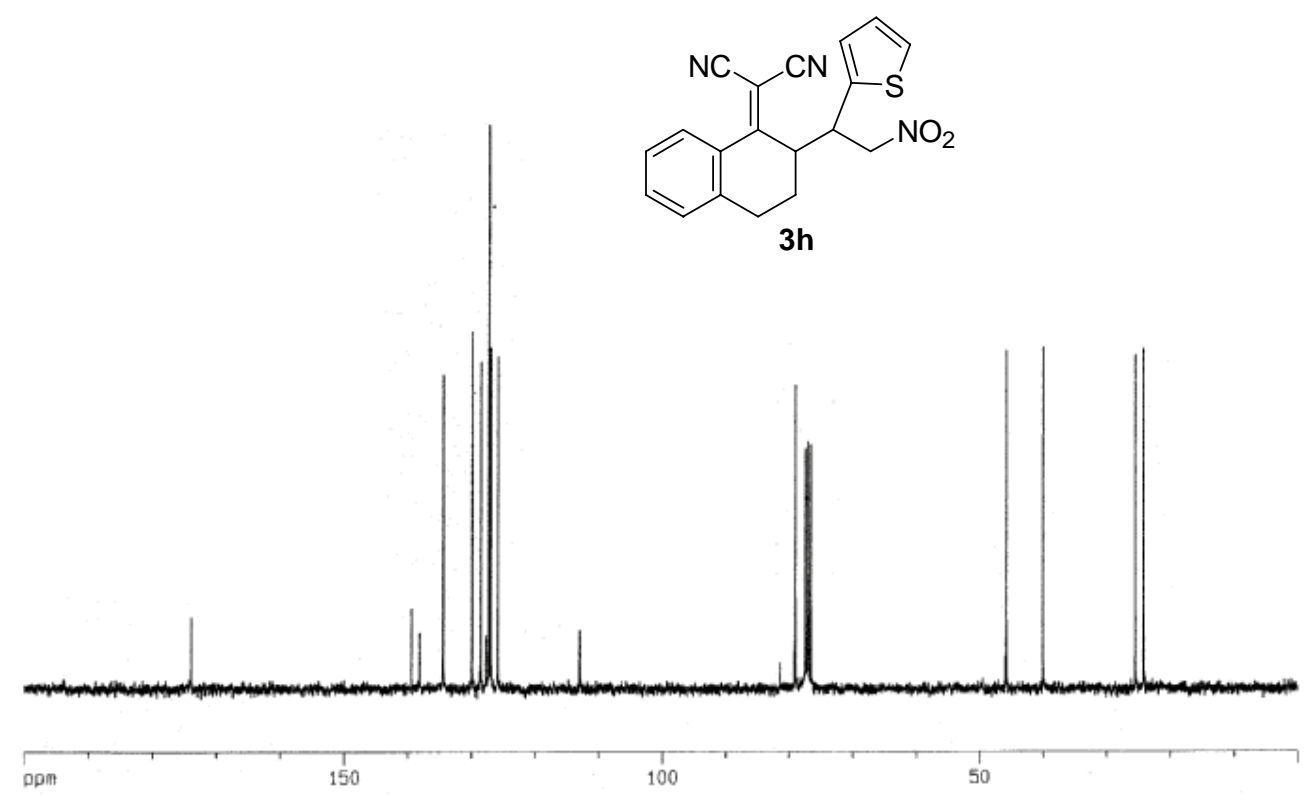

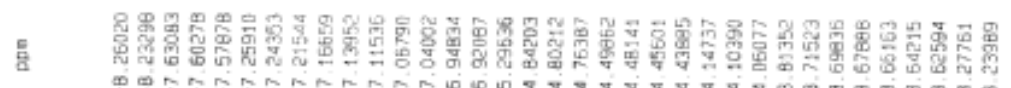
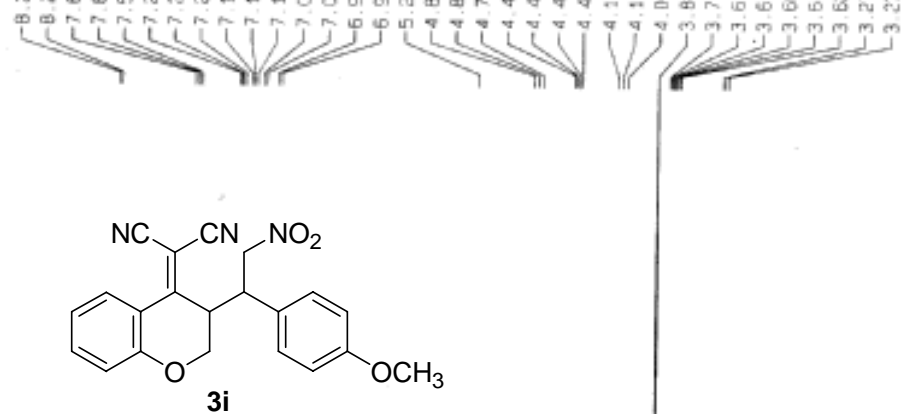

$3 i$

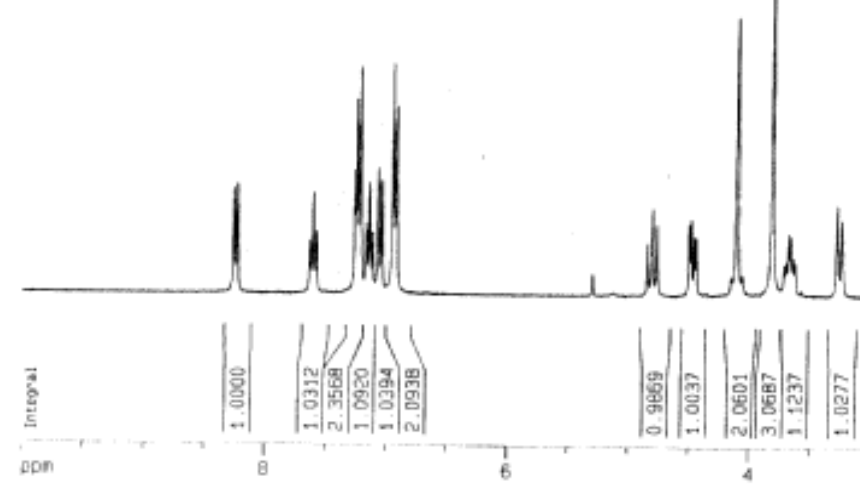




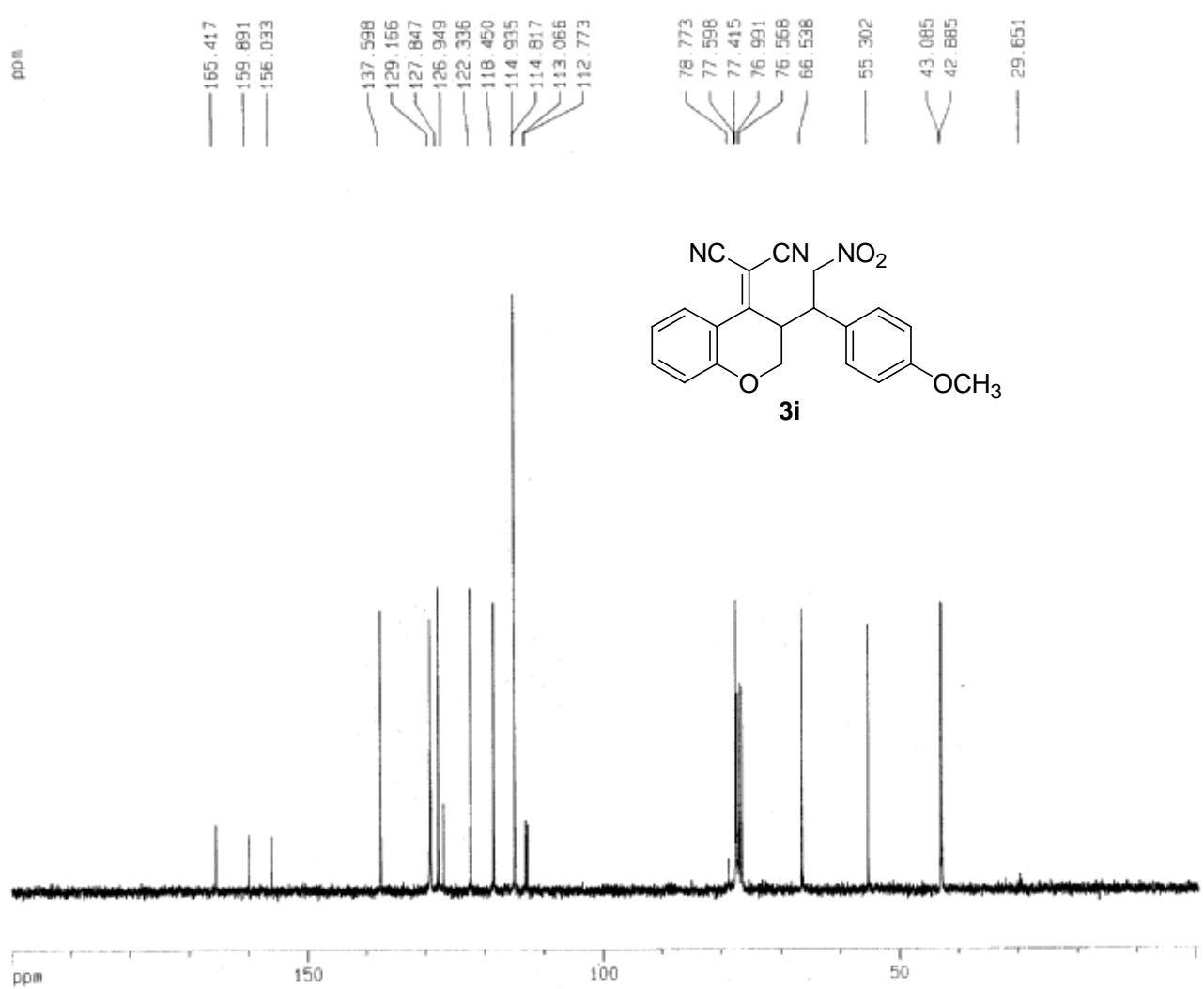

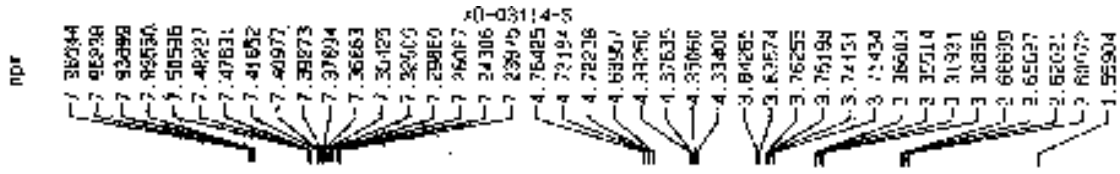

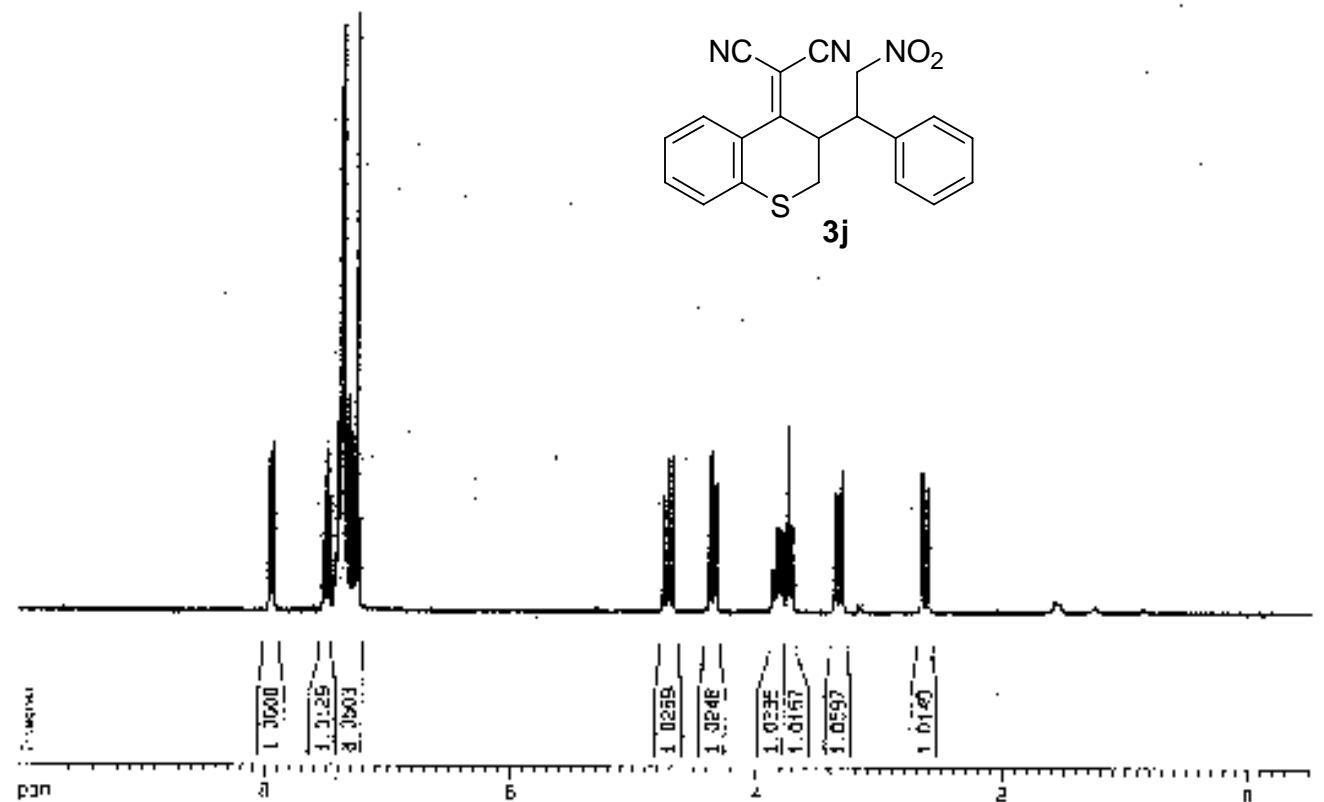




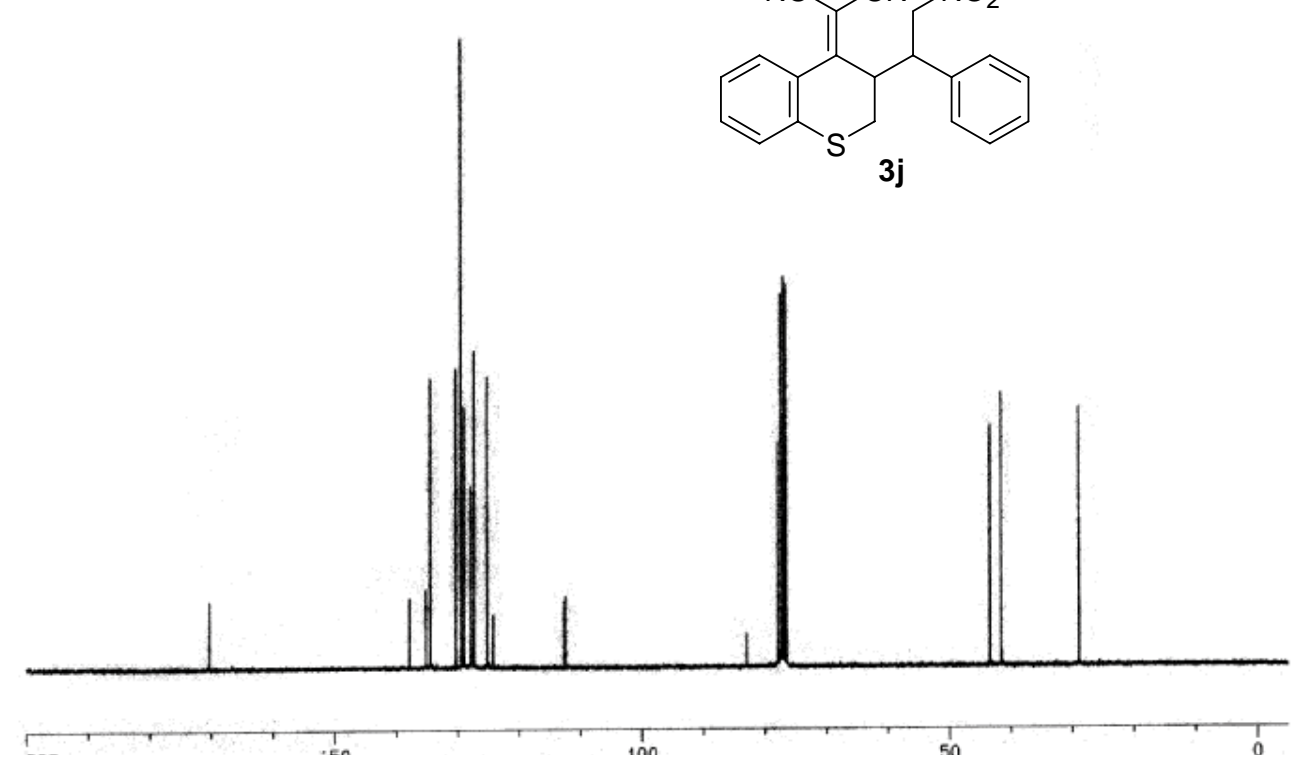

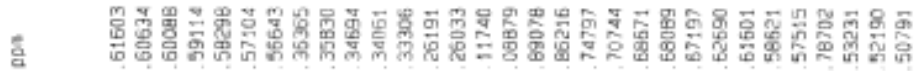

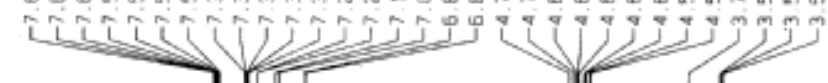<smiles>COc1ccc(C(C(C)N=O)C(C)C(=C(C#N)C#N)c2ccccc2)cc1</smiles>

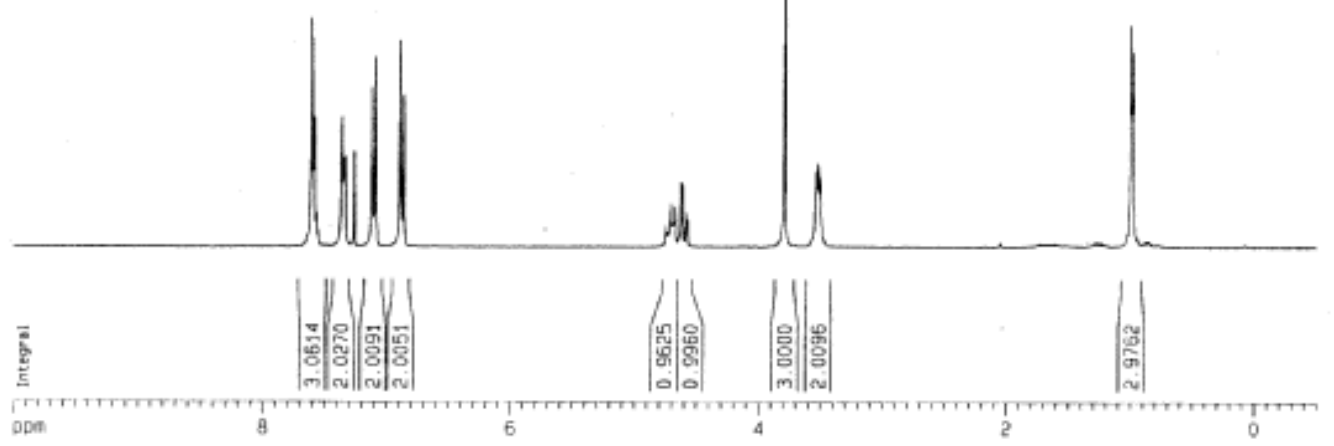



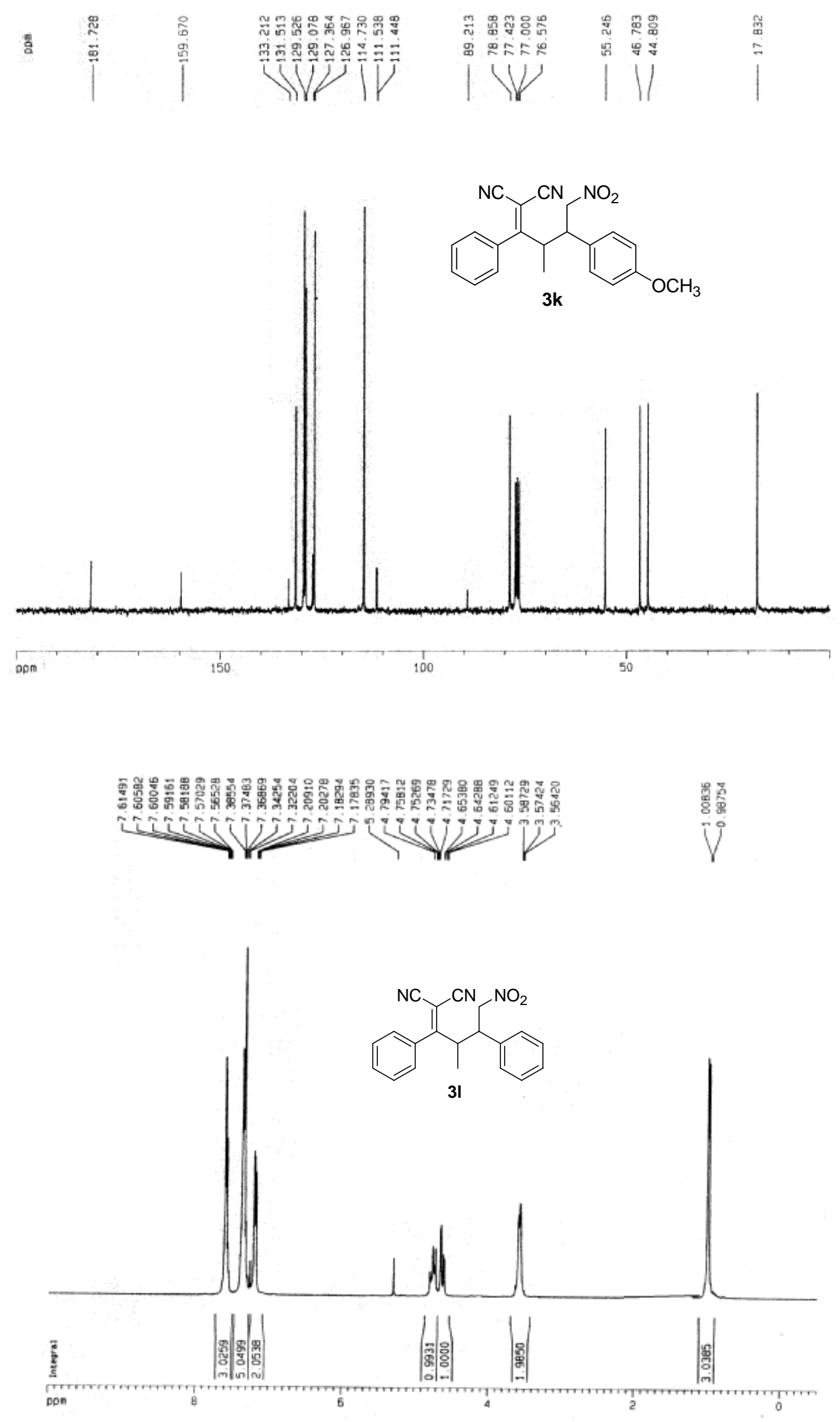


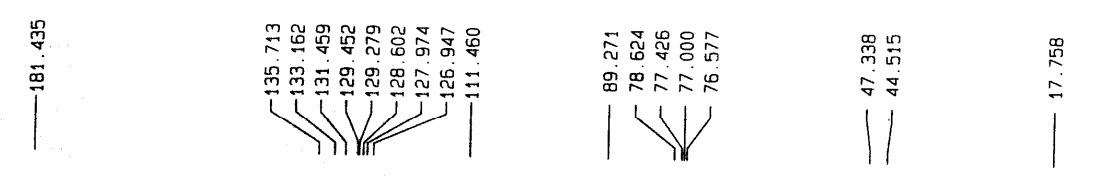
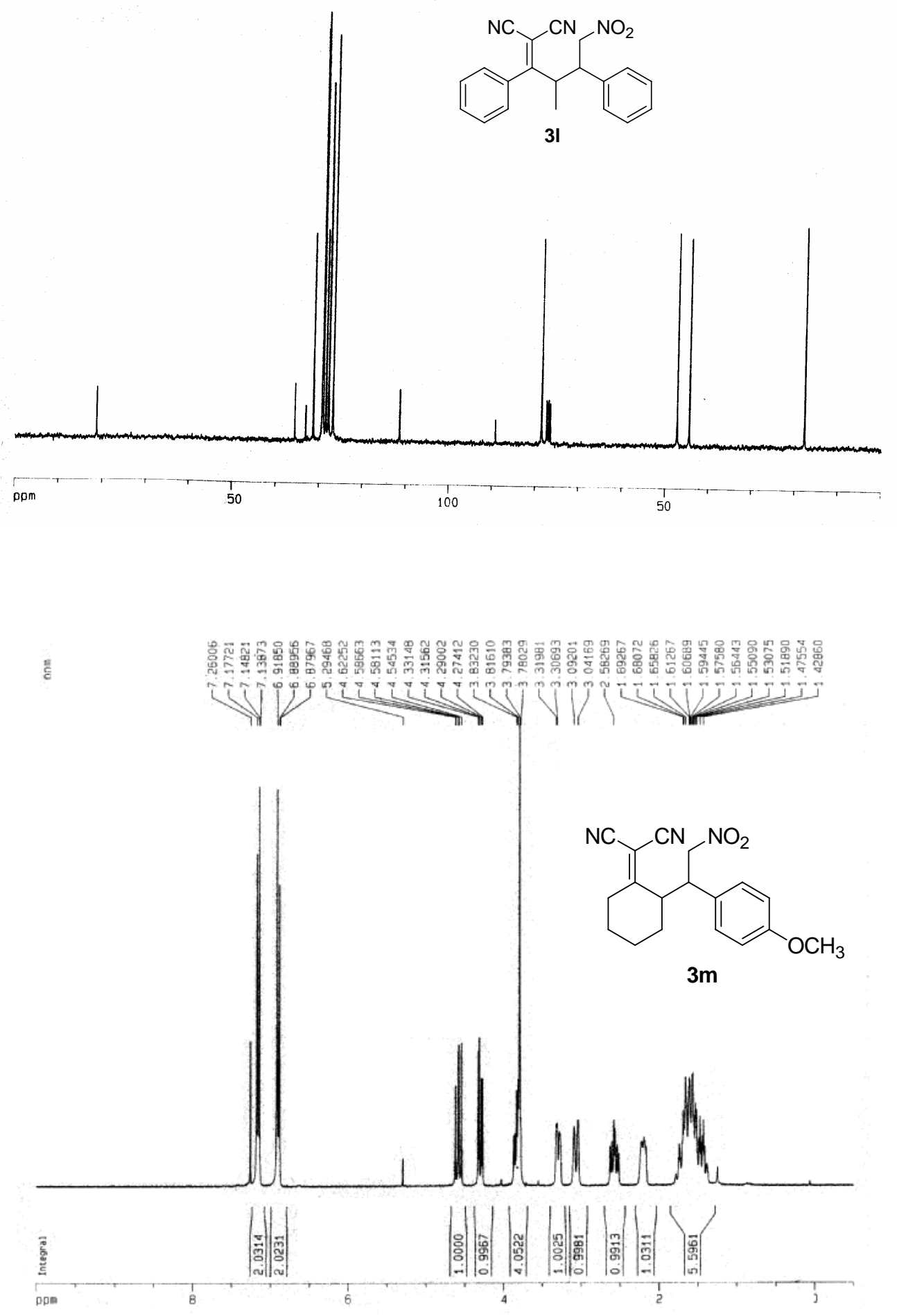

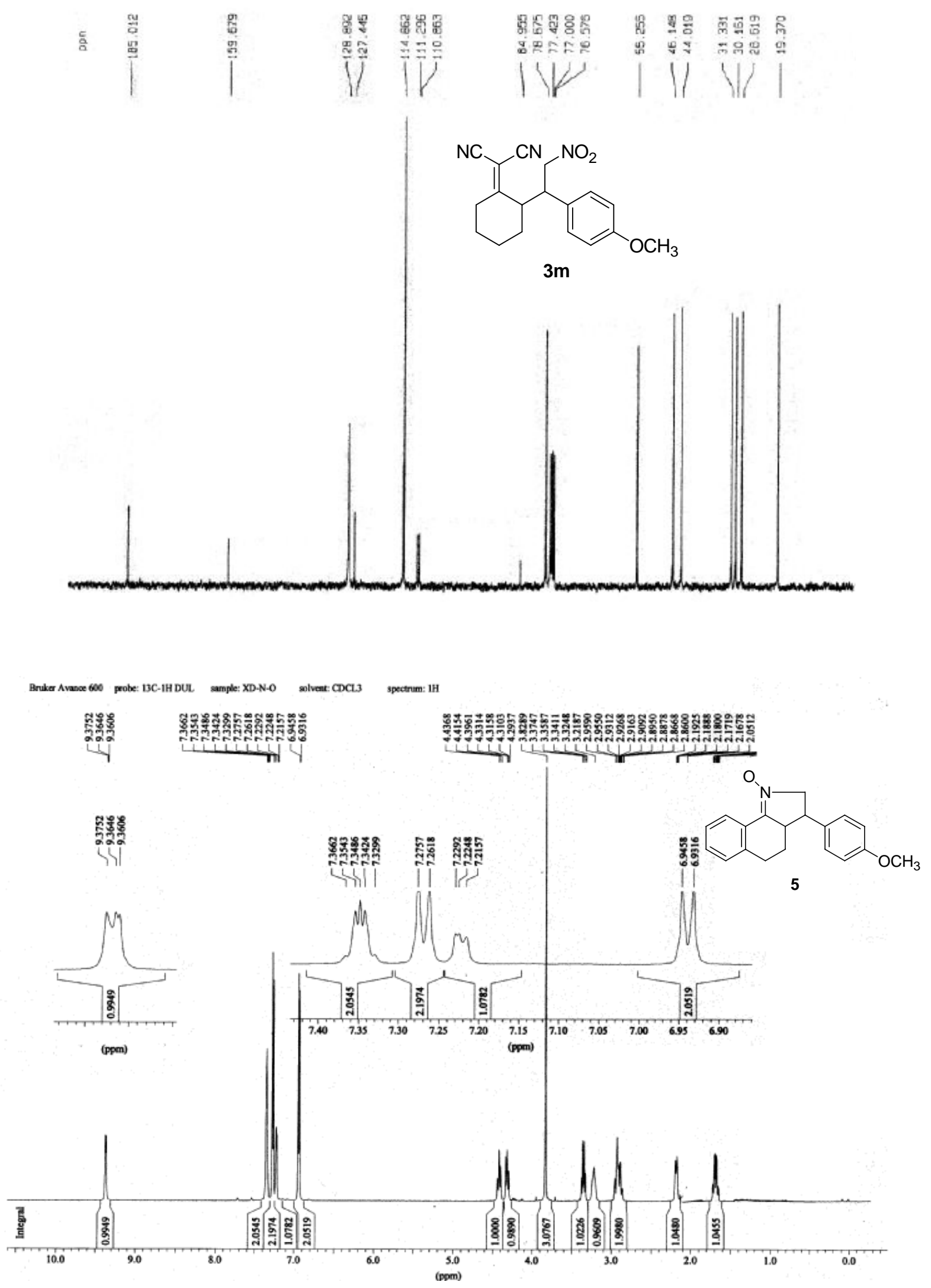


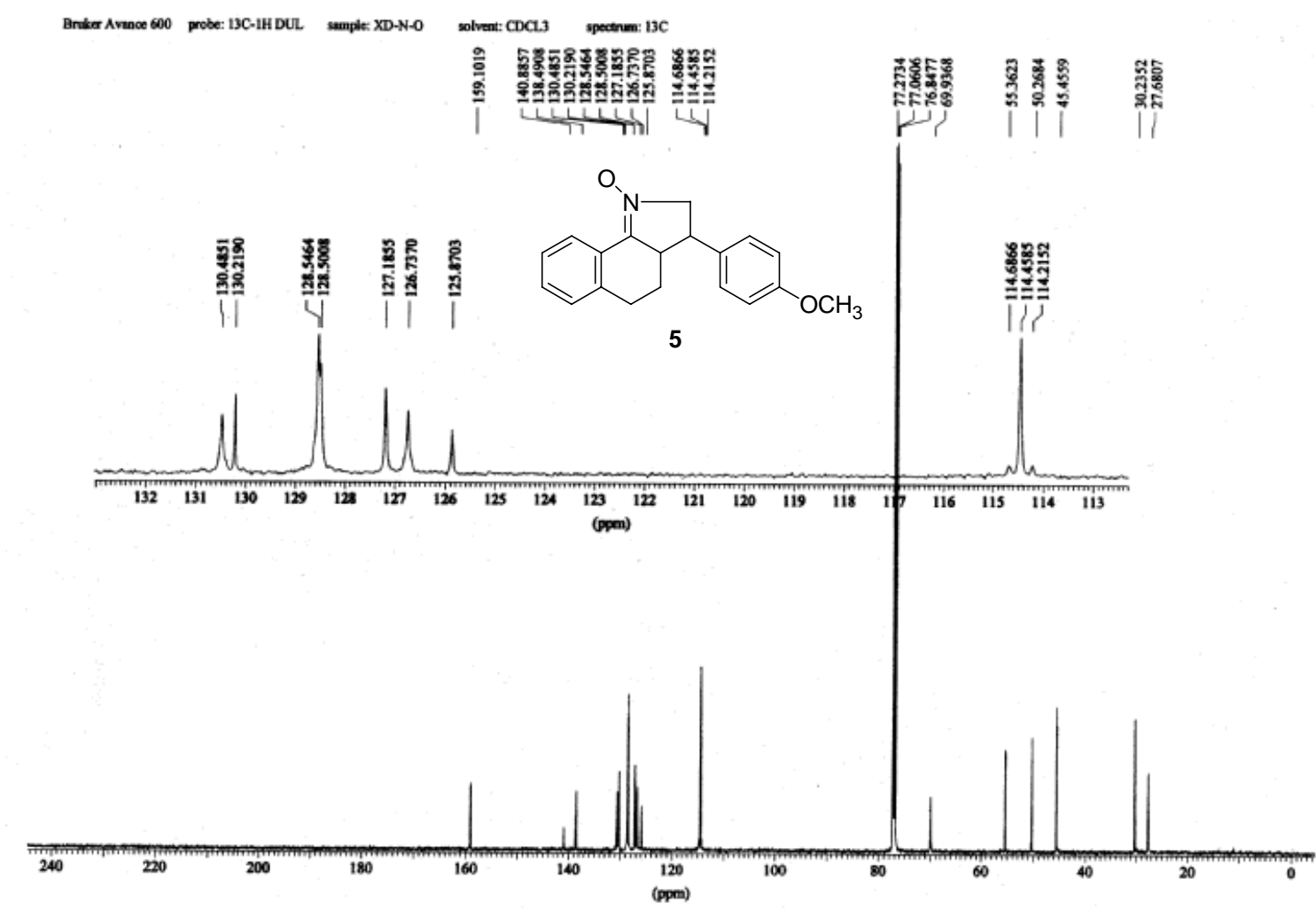

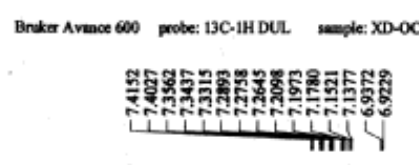

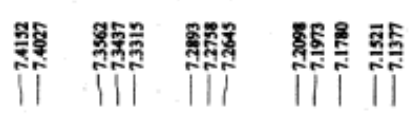
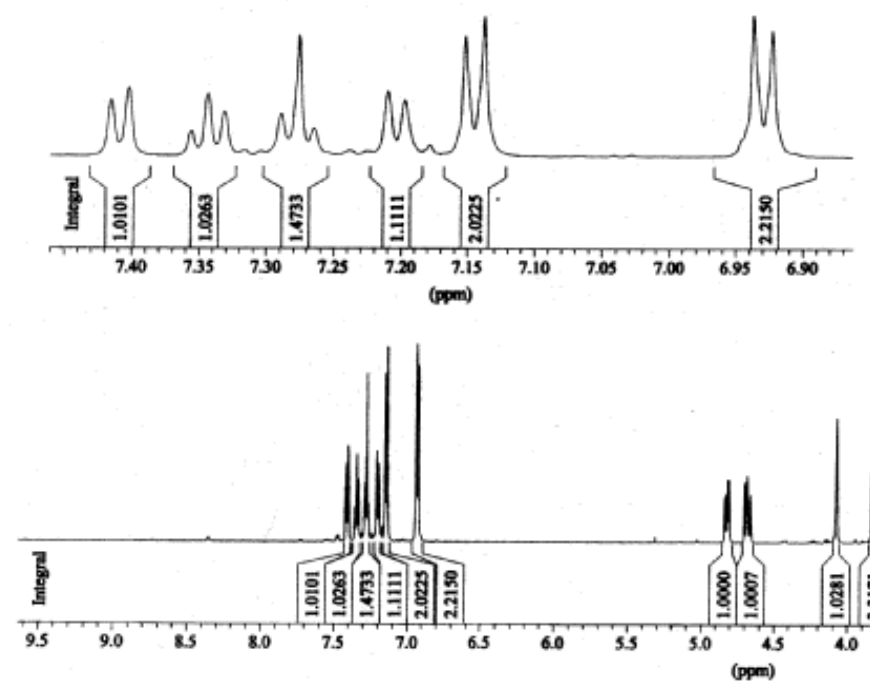

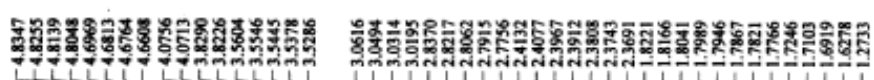

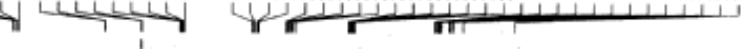

縭

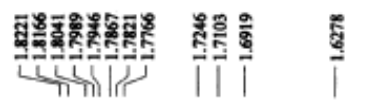

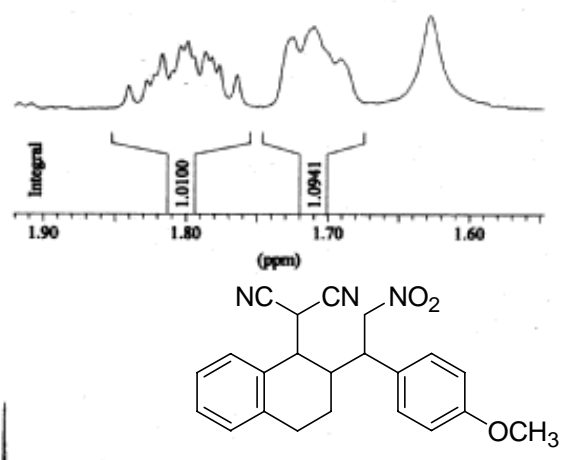



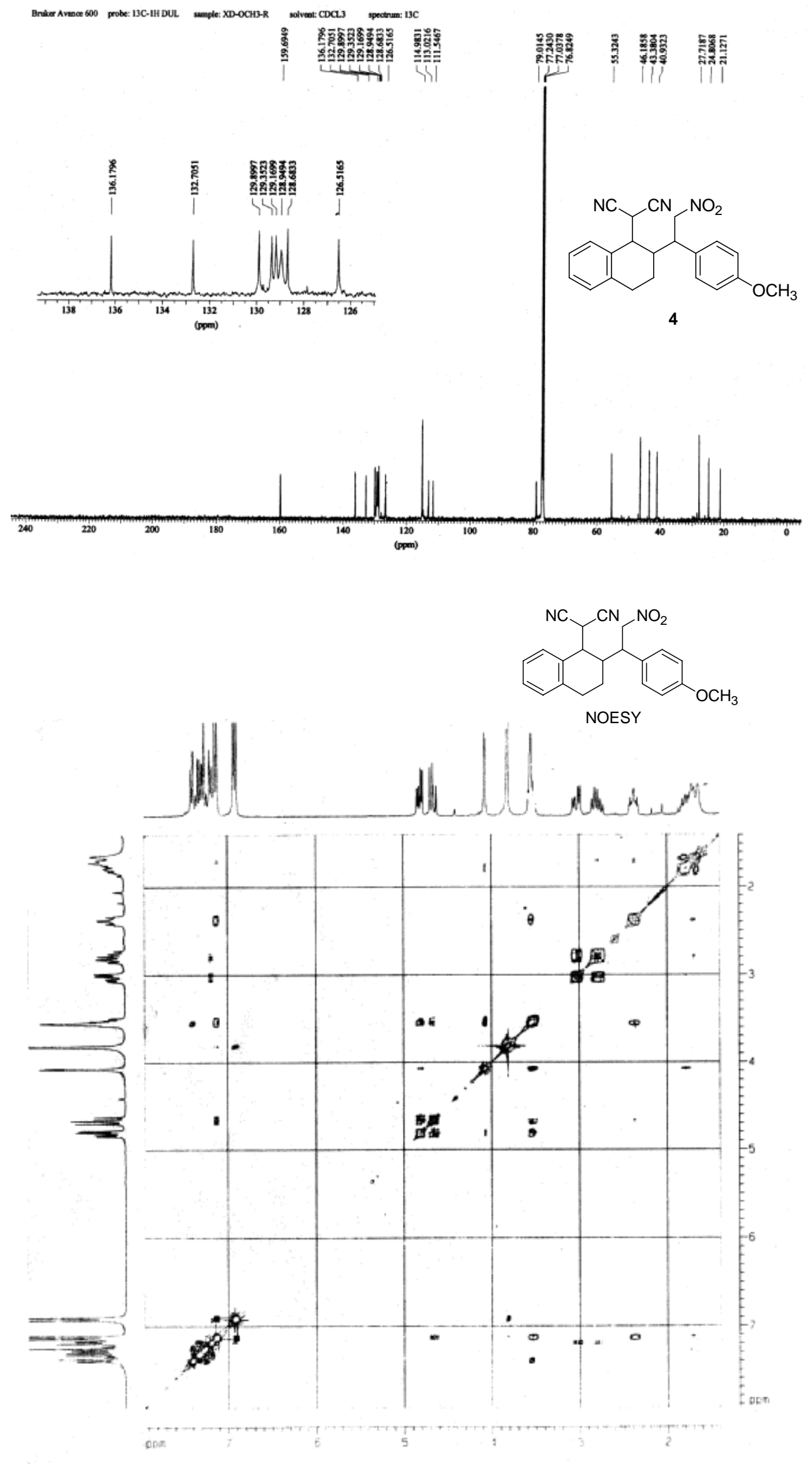

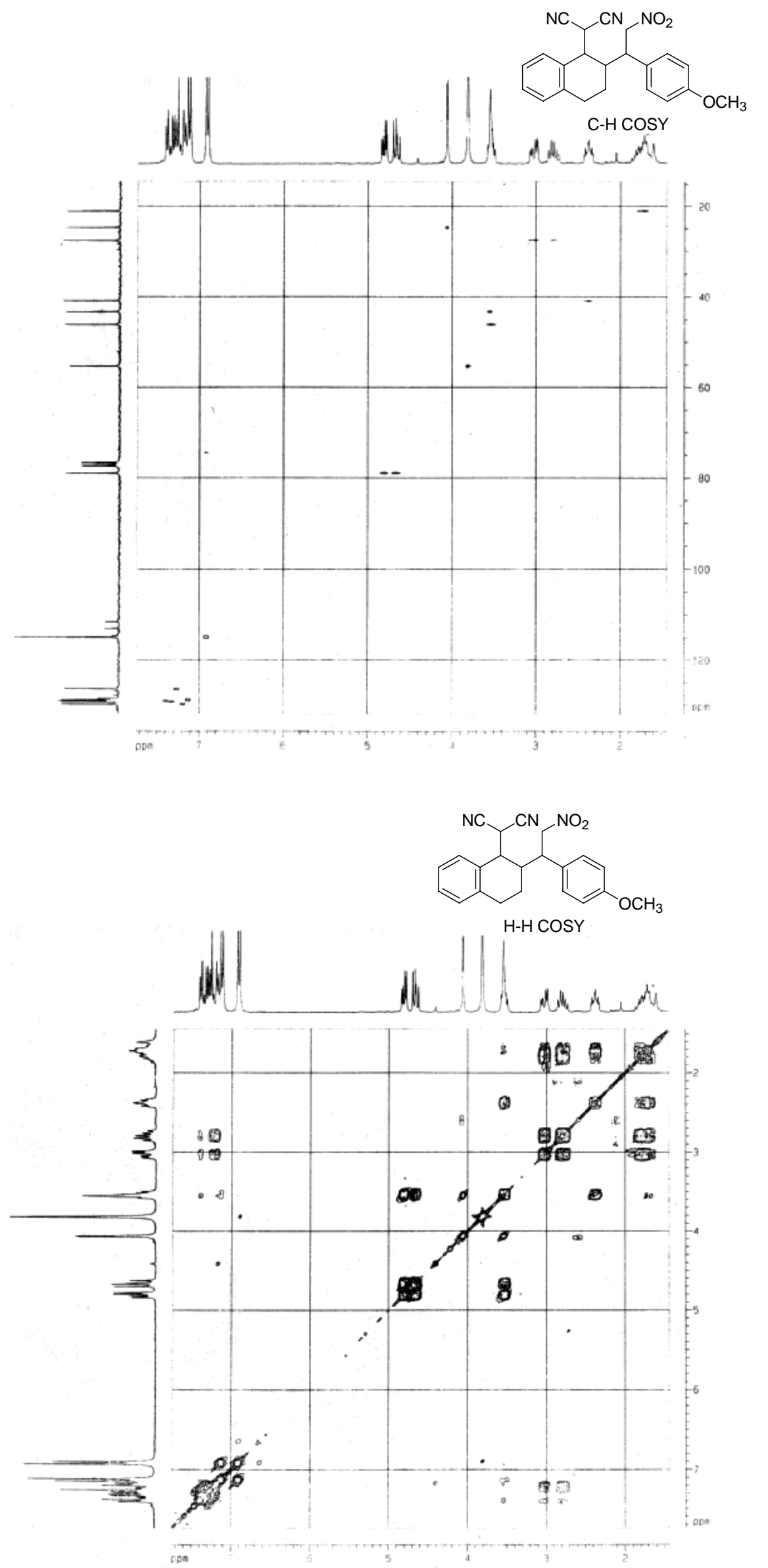


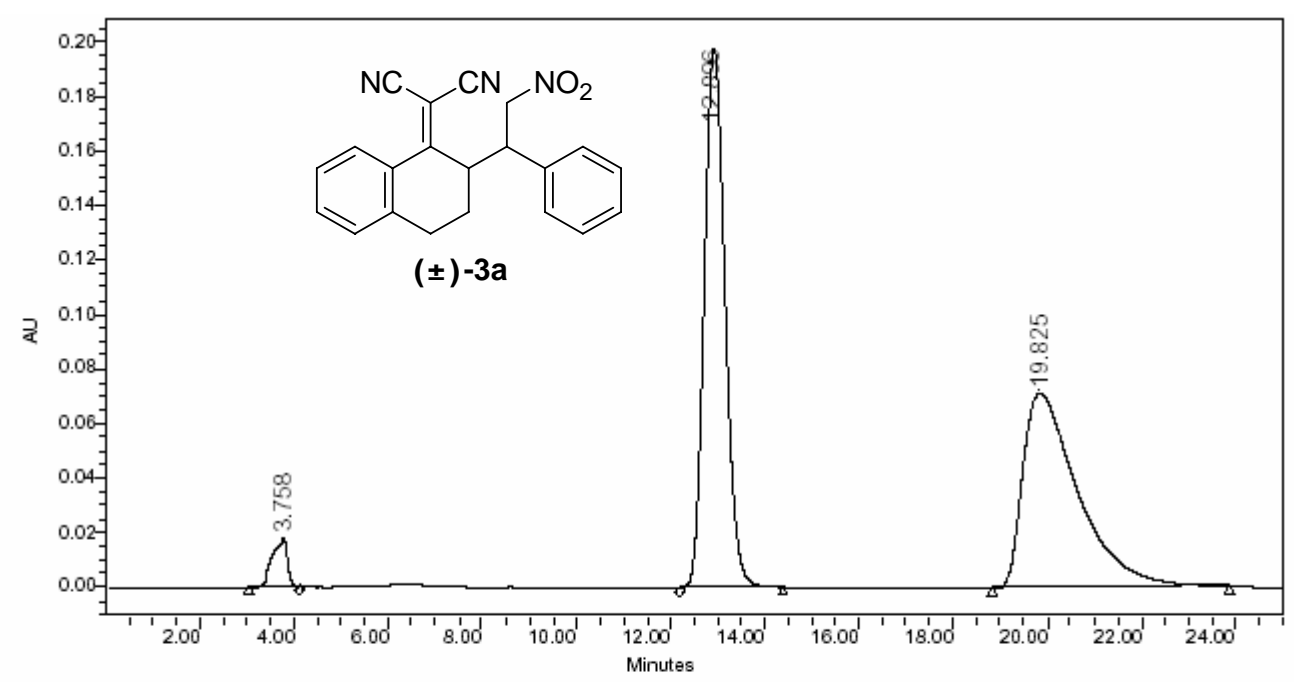

\begin{tabular}{|c|c|r|r|r|r|}
\hline & $\begin{array}{c}\text { RT } \\
(\mathrm{min})\end{array}$ & $\begin{array}{c}\text { Area } \\
\left(\mathrm{V}^{*} \mathrm{sec}\right)\end{array}$ & \% Area & $\begin{array}{l}\text { Height } \\
(\mathrm{V})\end{array}$ & $\begin{array}{c}\% \\
\text { Height }\end{array}$ \\
\hline 1 & 3.758 & 401271 & 3.26 & 17882 & 6.22 \\
\hline 2 & 12.896 & 6054855 & 49.15 & 197793 & 68.83 \\
\hline 3 & 19.825 & 5864150 & 47.60 & 71689 & 24.95 \\
\hline
\end{tabular}

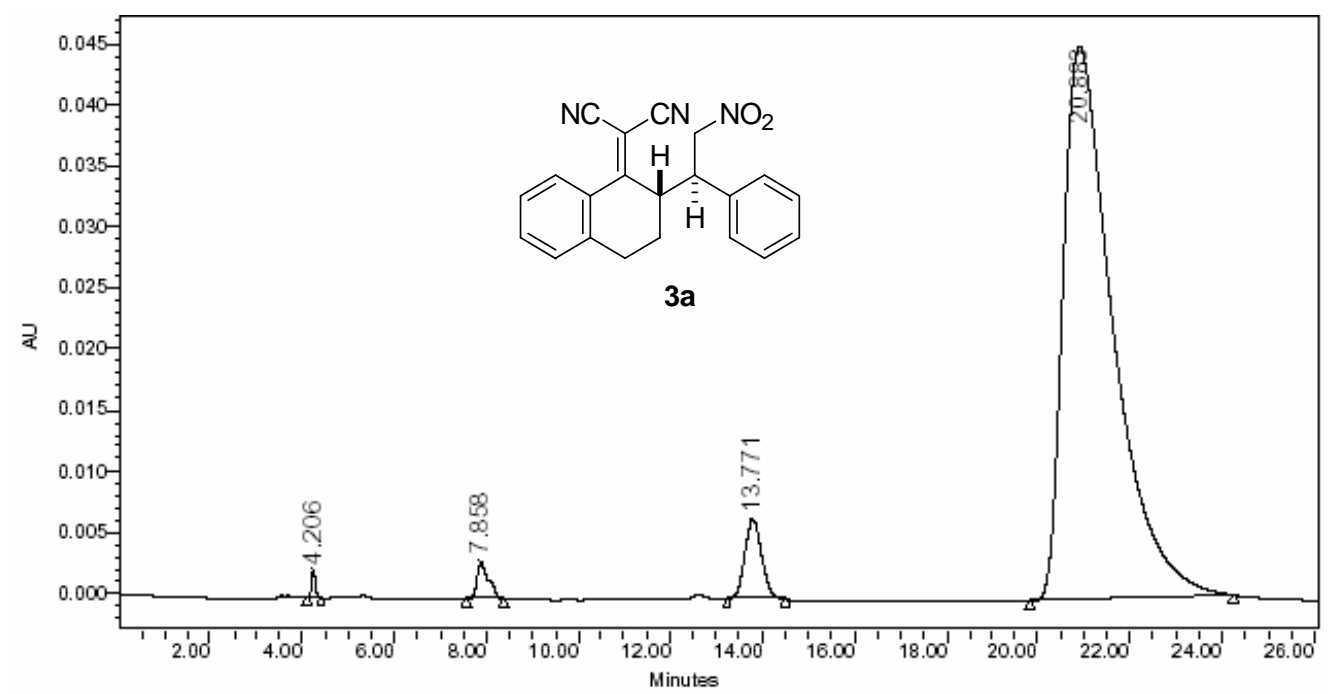

\begin{tabular}{|r|r|r|r|r|r|}
\hline & $\begin{array}{c}\text { RT } \\
(\mathrm{min})\end{array}$ & $\begin{array}{c}\text { Area } \\
\left(\mathrm{V}^{*} \mathrm{sec}\right)\end{array}$ & \% Area & $\begin{array}{c}\text { Height } \\
(\mathrm{V})\end{array}$ & $\begin{array}{c}\% \\
\text { Height }\end{array}$ \\
\hline 1 & 4.206 & 13268 & 0.36 & 2340 & 4.08 \\
\hline 2 & 7.858 & 55023 & 1.51 & 3096 & 5.40 \\
\hline 3 & 13.771 & 169840 & 4.66 & 6557 & 11.44 \\
\hline 4 & 20.883 & 3407526 & 93.47 & 45313 & 79.07 \\
\hline
\end{tabular}




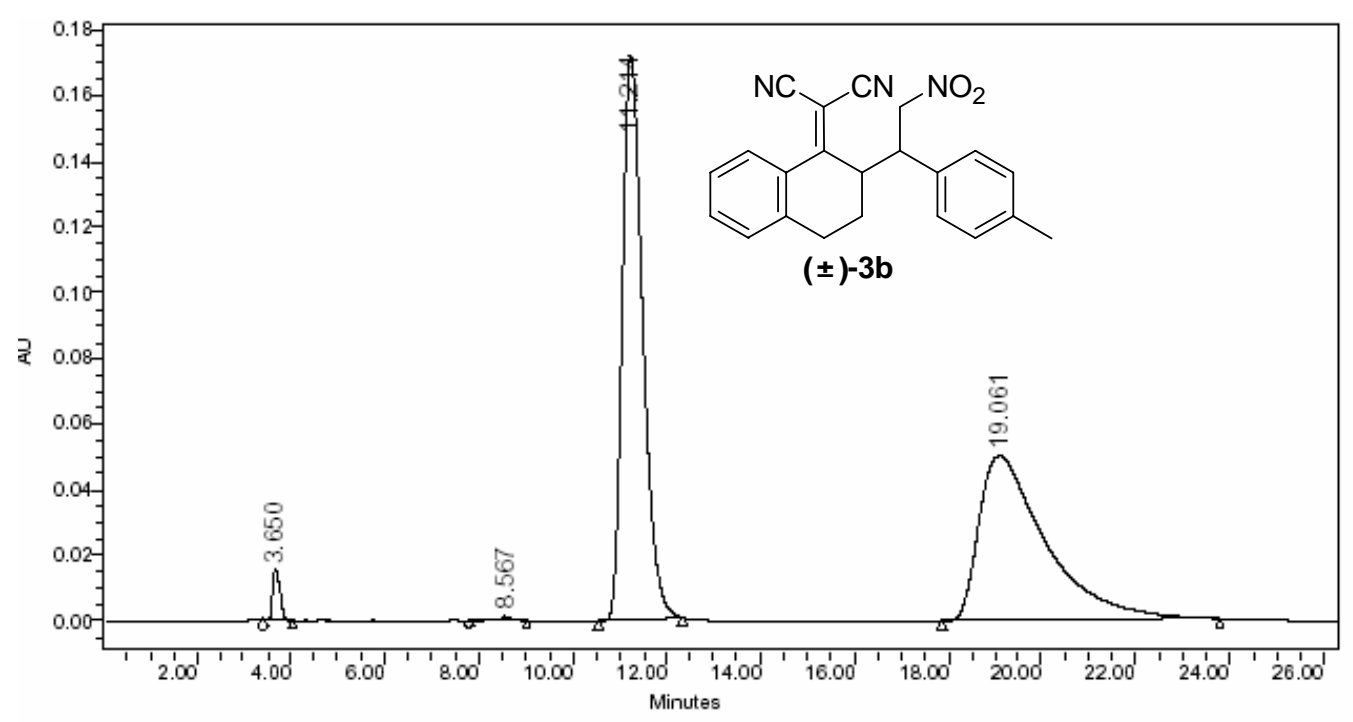

\begin{tabular}{|r|r|r|r|r|r|}
\hline & $\begin{array}{c}\text { RT } \\
(\mathrm{min})\end{array}$ & $\begin{array}{c}\text { Area } \\
\left(\mathrm{V}^{*} \text { sec }\right)\end{array}$ & \% Area & $\begin{array}{l}\text { Height } \\
(\mathrm{V})\end{array}$ & $\begin{array}{c}\% \\
\text { Height }\end{array}$ \\
\hline 1 & 3.650 & 177322 & 1.70 & 15398 & 6.45 \\
\hline 2 & 8.567 & 29674 & 0.29 & 1152 & 0.48 \\
\hline 3 & 11.214 & 5202209 & 49.96 & 172040 & 72.01 \\
\hline 4 & 19.061 & 5002653 & 48.05 & 50313 & 21.06 \\
\hline
\end{tabular}

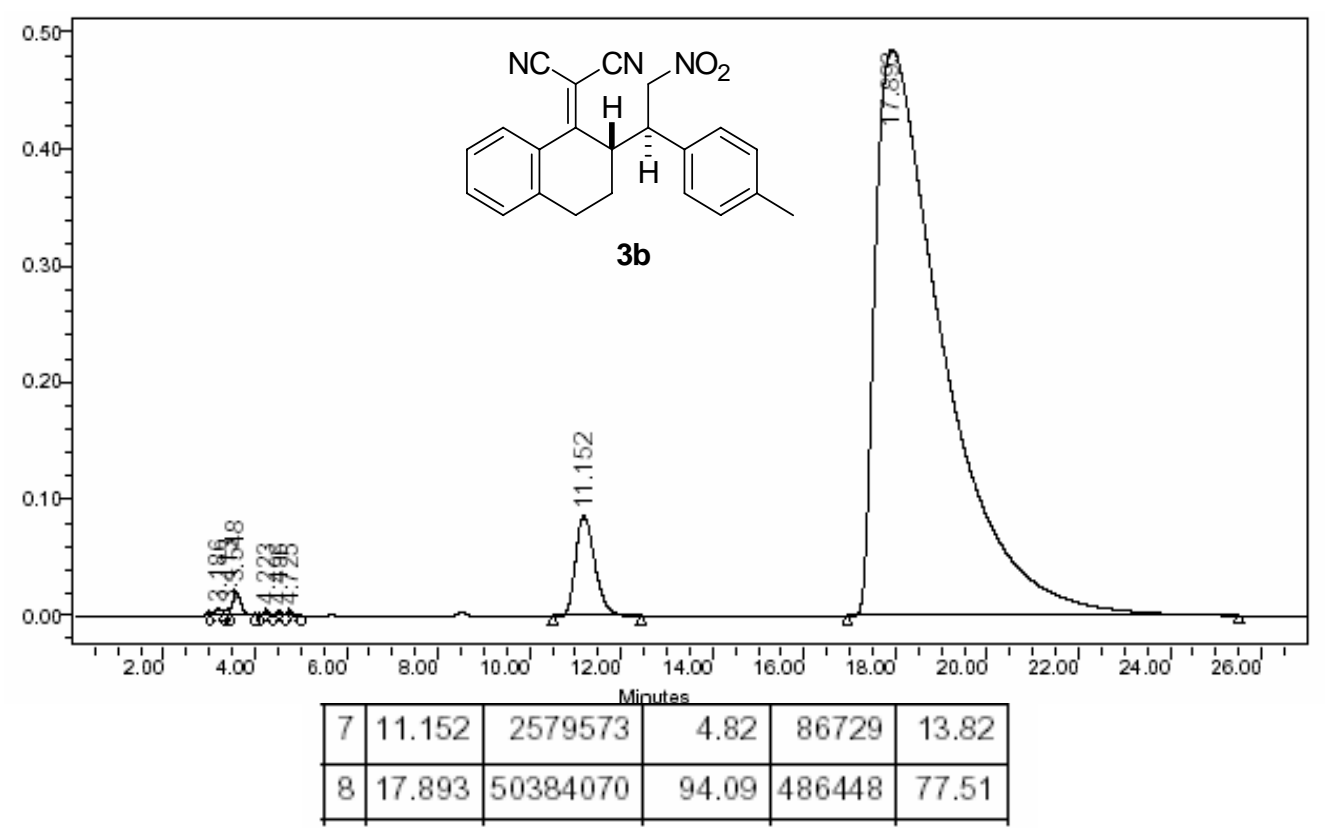




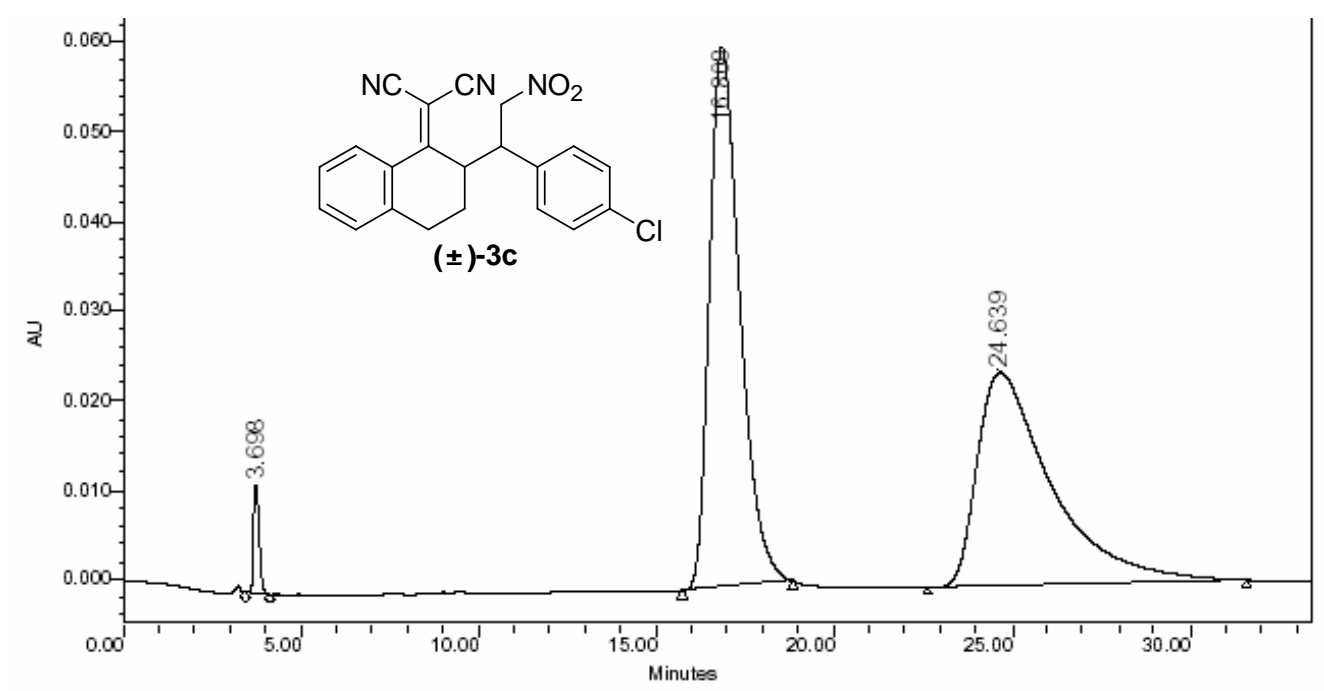

\begin{tabular}{|c|c|c|r|r|c|}
\hline & $\begin{array}{c}\text { RT } \\
(\text { min })\end{array}$ & $\begin{array}{c}\text { Area } \\
\left(V^{*} \text { sec }\right)\end{array}$ & \% Area & $\begin{array}{c}\text { Height } \\
(\mathrm{V})\end{array}$ & $\begin{array}{c}\% \\
\text { Height }\end{array}$ \\
\hline 1 & 3.698 & 139736 & 1.93 & 12121 & 12.58 \\
\hline 2 & 16.808 & 3606535 & 49.92 & 60358 & 62.64 \\
\hline 3 & 24.639 & 3478769 & 48.15 & 23871 & 24.78 \\
\hline
\end{tabular}

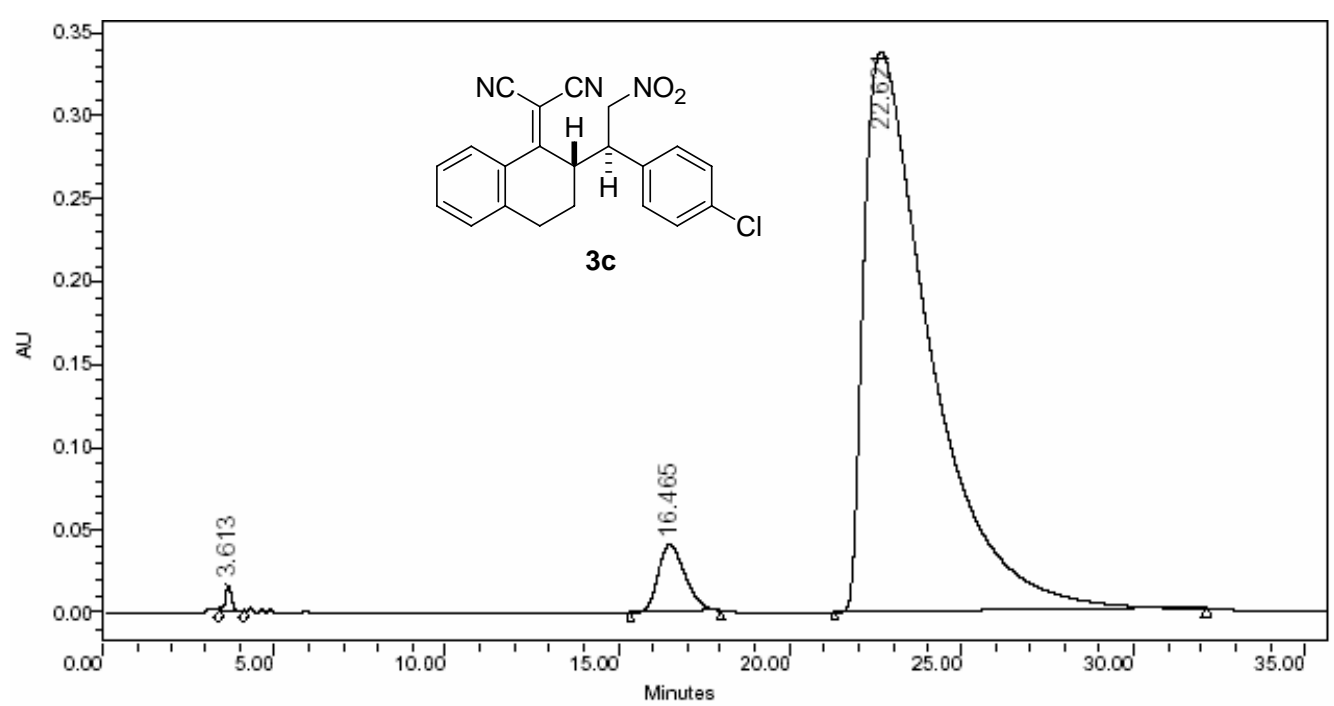

\begin{tabular}{|l|r|r|r|r|r|}
\hline & $\begin{array}{c}\text { RT } \\
(\mathrm{min})\end{array}$ & $\begin{array}{c}\text { Area } \\
\left(\mathrm{V}^{\star} \mathrm{sec}\right)\end{array}$ & $\%$ Area & $\begin{array}{c}\text { Height } \\
(\mathrm{V})\end{array}$ & $\begin{array}{c}\% \\
\text { Height }\end{array}$ \\
\hline 1 & 3.613 & 249837 & 0.52 & 16662 & 4.21 \\
\hline 2 & 16.465 & 2254917 & 4.66 & 40840 & 10.31 \\
\hline 3 & 22.621 & 45839289 & 94.82 & 338437 & 85.48 \\
\hline
\end{tabular}




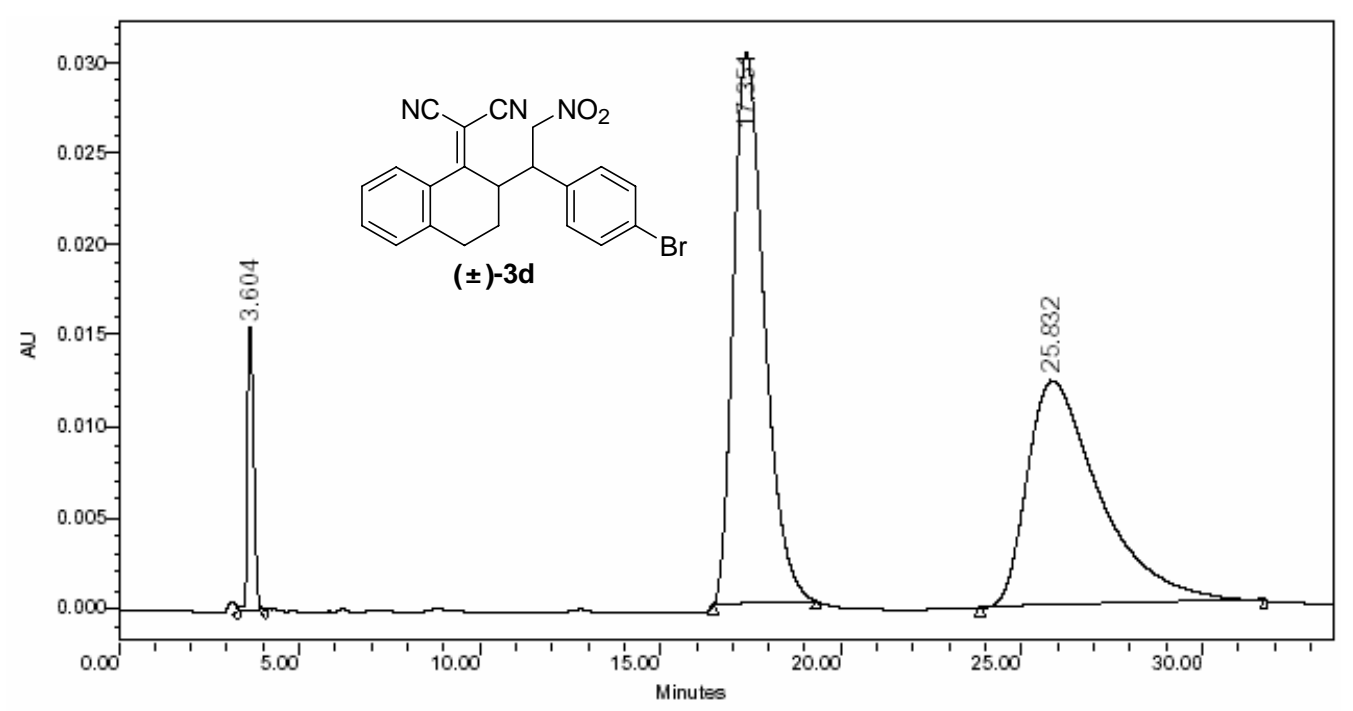

\begin{tabular}{|c|c|c|r|c|c|}
\hline & $\begin{array}{c}\text { RT } \\
(\mathrm{min})\end{array}$ & $\begin{array}{c}\text { Area } \\
\left(\mathrm{V}^{*} \text { sec }\right)\end{array}$ & $\%$ Area & $\begin{array}{c}\text { Height } \\
(\mathrm{V})\end{array}$ & $\begin{array}{c}\% \\
\text { Height }\end{array}$ \\
\hline 1 & 3.604 & 191779 & 5.08 & 15677 & 26.82 \\
\hline 2 & 17.351 & 1817379 & 48.18 & 30380 & 51.98 \\
\hline 3 & 25.832 & 1763019 & 46.74 & 12394 & 21.20 \\
\hline
\end{tabular}

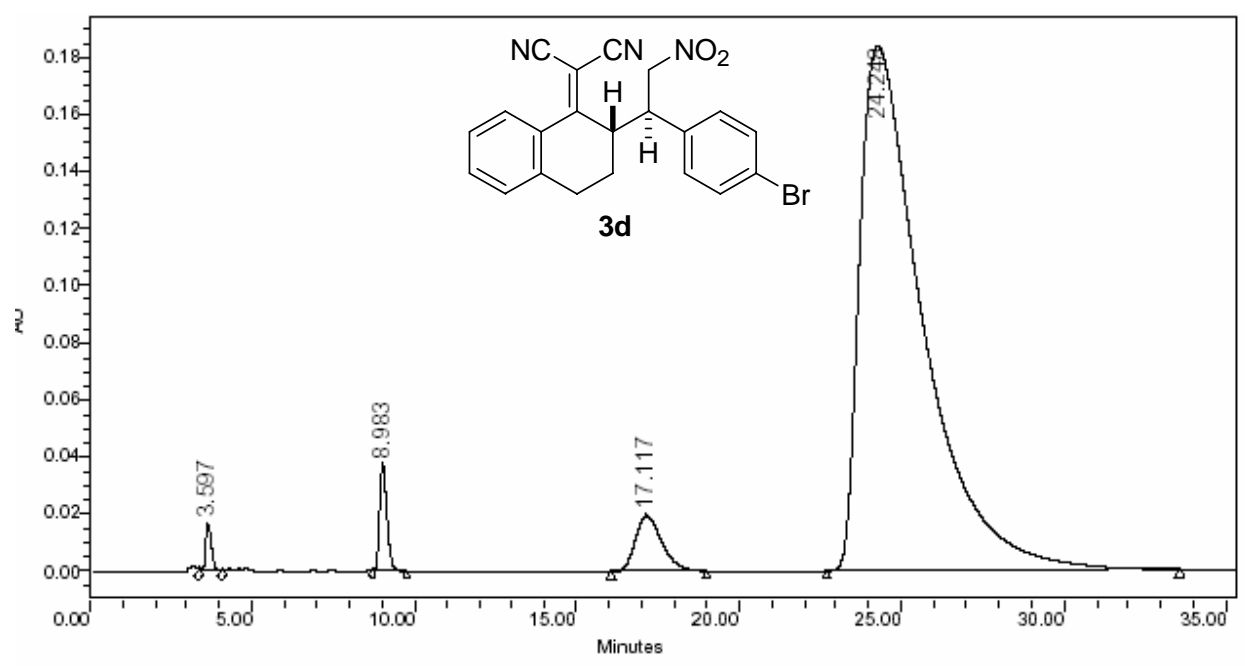

\begin{tabular}{|r|r|r|r|r|r|}
\hline & $\begin{array}{c}\text { RT } \\
(\mathrm{min})\end{array}$ & $\begin{array}{c}\text { Area } \\
\left(\mathrm{V}^{*} \mathrm{sec}\right)\end{array}$ & $\%$ Area & $\begin{array}{c}\text { Height } \\
(\mathrm{V})\end{array}$ & $\begin{array}{c}\% \\
\text { Height }\end{array}$ \\
\hline 1 & 3.597 & 227038 & 0.84 & 16693 & 6.48 \\
\hline 2 & 8.983 & 591800 & 2.20 & 37540 & 14.57 \\
\hline 3 & 17.117 & 1131150 & 4.21 & 19625 & 7.62 \\
\hline 4 & 24.248 & 24919636 & 92.74 & 183756 & 71.33 \\
\hline
\end{tabular}




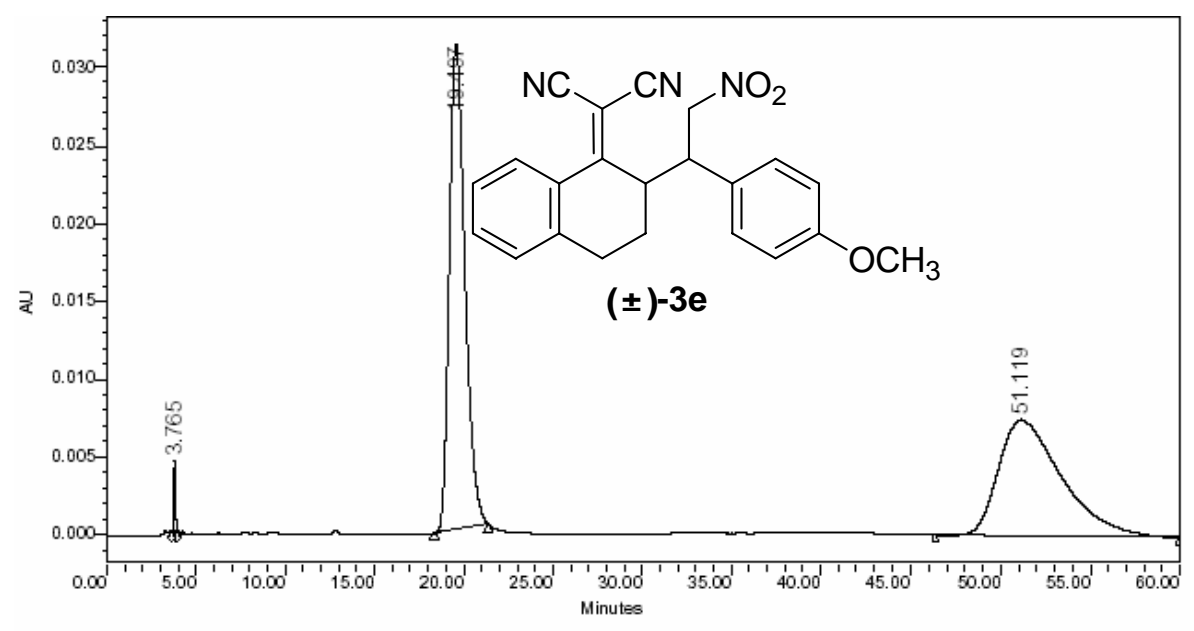

\begin{tabular}{|l|c|r|r|r|c|}
\hline & $\begin{array}{c}\text { RT } \\
(\mathrm{min})\end{array}$ & $\begin{array}{c}\text { Area } \\
\left(\mathrm{V}^{*} \text { sec) }\right.\end{array}$ & \% Area & $\begin{array}{c}\text { Height } \\
\text { (V) }\end{array}$ & $\begin{array}{c}\% \\
\text { Height }\end{array}$ \\
\hline 1 & 3.765 & 28397 & 0.75 & 4677 & 10.79 \\
\hline 2 & 19.497 & 1896303 & 50.17 & 31216 & 72.05 \\
\hline 3 & 51.119 & 1854857 & 49.08 & 7435 & 17.16 \\
\hline
\end{tabular}

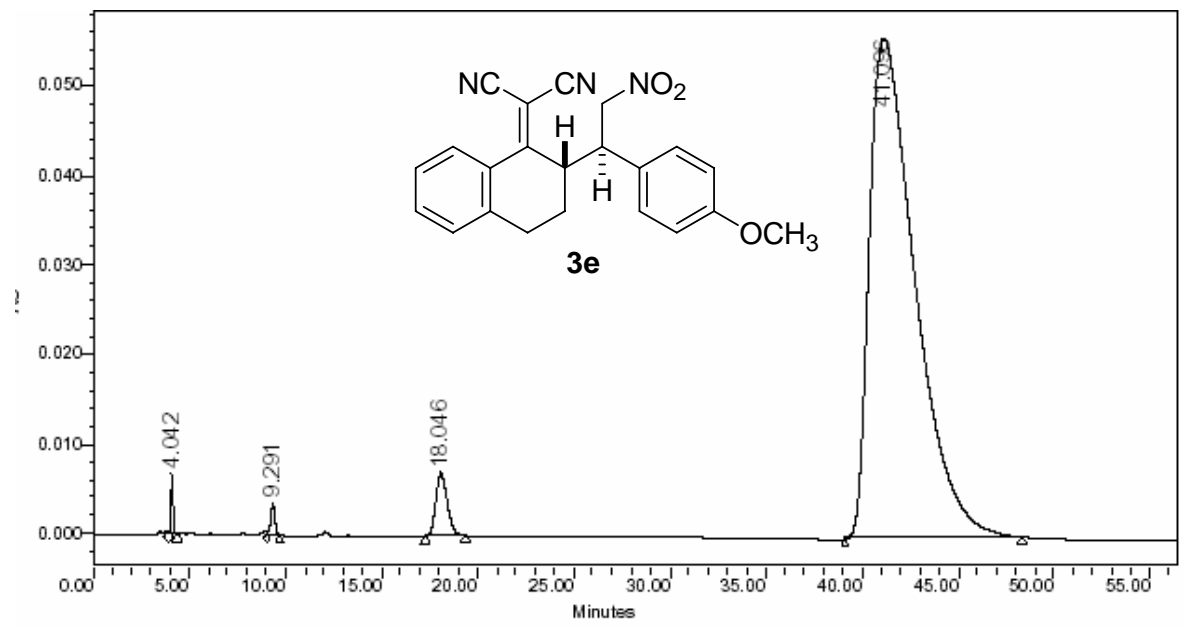

\begin{tabular}{|r|r|r|r|r|r|}
\hline & $\begin{array}{c}\text { RT } \\
(\mathrm{min})\end{array}$ & $\begin{array}{c}\text { Area } \\
\left(\mathrm{V}^{*} \mathrm{sec}\right)\end{array}$ & \% Area & $\begin{array}{l}\text { Height } \\
(\mathrm{V})\end{array}$ & $\begin{array}{c}\% \\
\text { Height }\end{array}$ \\
\hline 1 & 4.042 & 46171 & 0.48 & 6761 & 9.26 \\
\hline 2 & 9.291 & 55060 & 0.57 & 3483 & 4.77 \\
\hline 3 & 18.046 & 290738 & 3.02 & 6951 & 9.52 \\
\hline 4 & 41.096 & 9236070 & 95.93 & 55838 & 76.46 \\
\hline
\end{tabular}




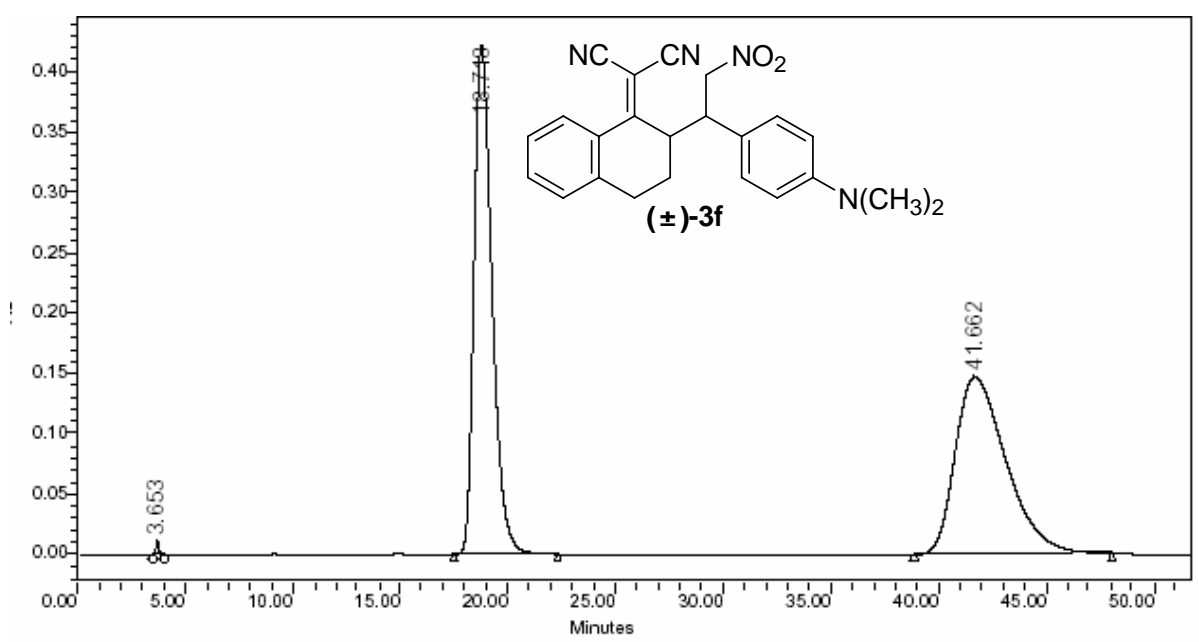

\begin{tabular}{|c|c|c|r|r|r|}
\hline & $\begin{array}{c}\text { RT } \\
(\text { min })\end{array}$ & $\begin{array}{c}\text { Area } \\
\left(V^{*} \text { sec }\right)\end{array}$ & $\%$ Area & $\begin{array}{c}\text { Height } \\
(\mathrm{V})\end{array}$ & $\begin{array}{c}\% \\
\text { Height }\end{array}$ \\
\hline 1 & 3.653 & 108047 & 0.22 & 11696 & 2.01 \\
\hline 2 & 18.718 & 24523289 & 50.20 & 421891 & 72.65 \\
\hline 3 & 41.662 & 24223792 & 49.58 & 147164 & 25.34 \\
\hline
\end{tabular}

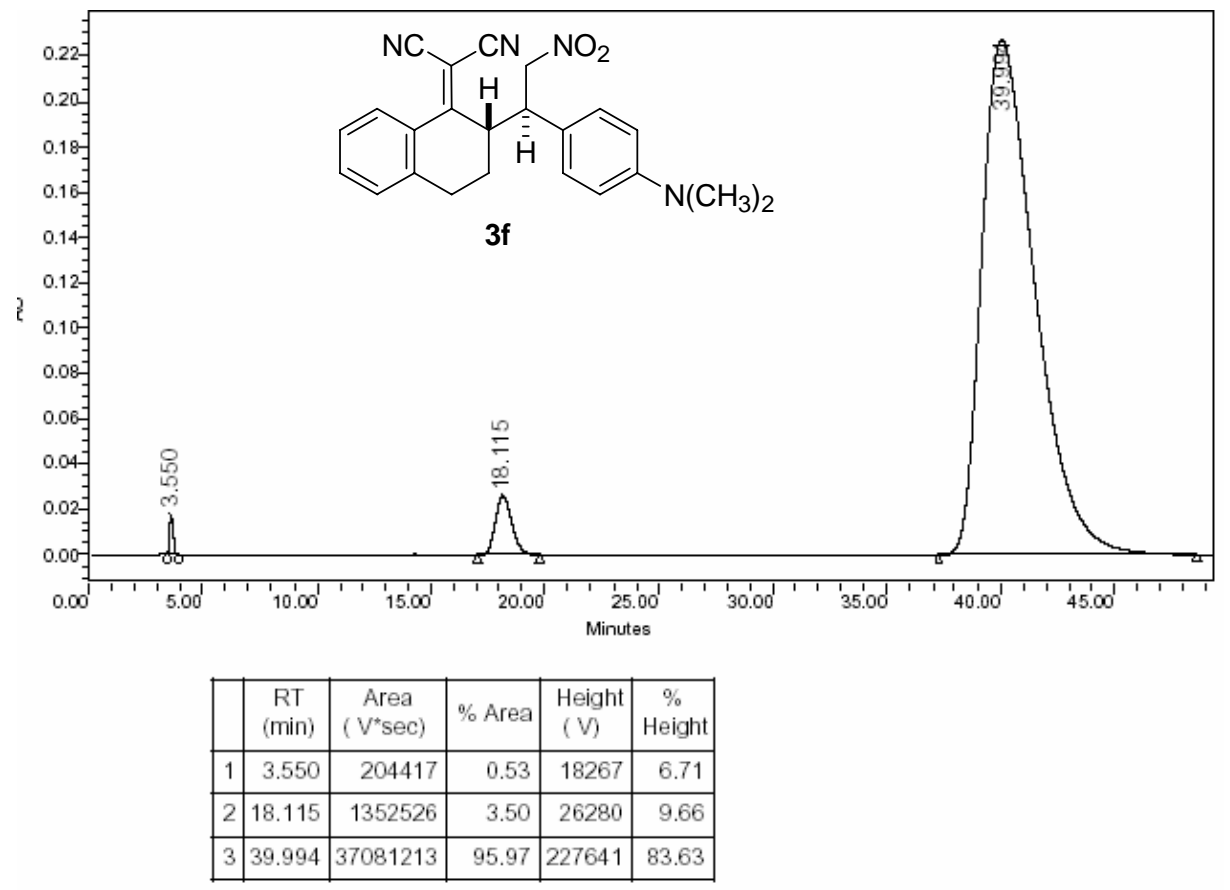




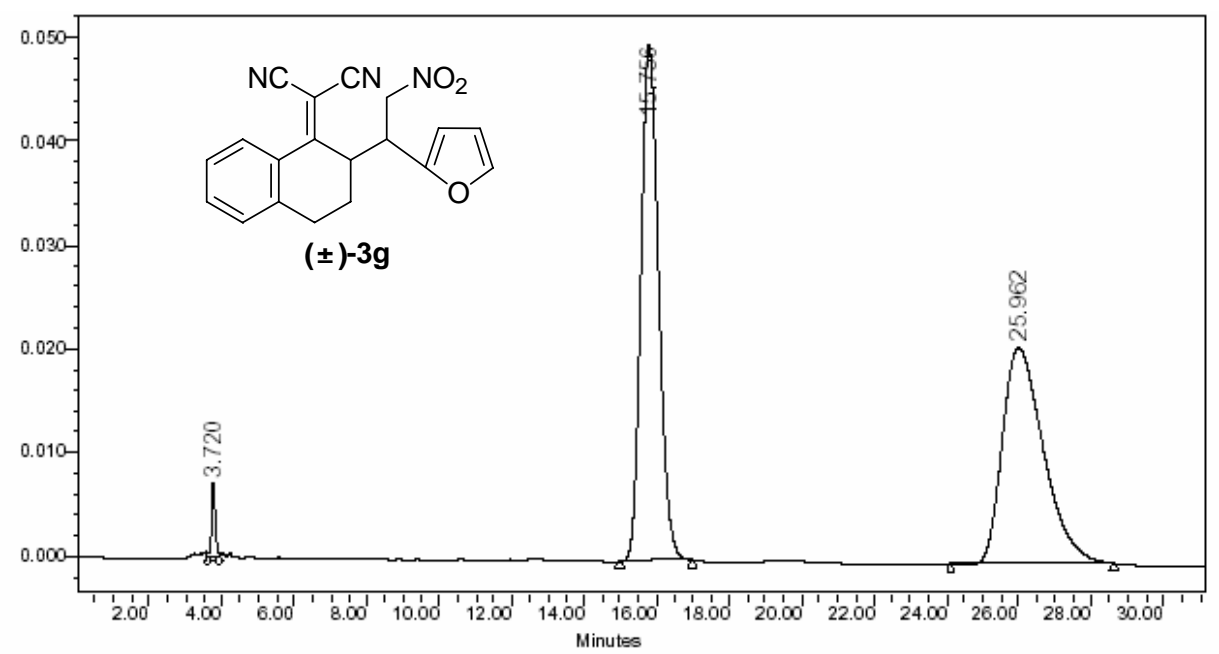

\begin{tabular}{|l|r|r|r|r|r|}
\hline & $\begin{array}{c}\text { RT } \\
(\mathrm{min})\end{array}$ & $\begin{array}{c}\text { Area } \\
\left(\mathrm{V}^{*} \mathrm{sec}\right)\end{array}$ & $\%$ Area & $\begin{array}{c}\text { Height } \\
(\mathrm{V})\end{array}$ & $\begin{array}{c}\% \\
\text { Height }\end{array}$ \\
\hline 1 & 3.720 & 50355 & 1.48 & 7223 & 9.25 \\
\hline 2 & 15.756 & 1687764 & 49.70 & 49886 & 63.90 \\
\hline 3 & 25.962 & 1657569 & 48.81 & 20957 & 26.85 \\
\hline
\end{tabular}

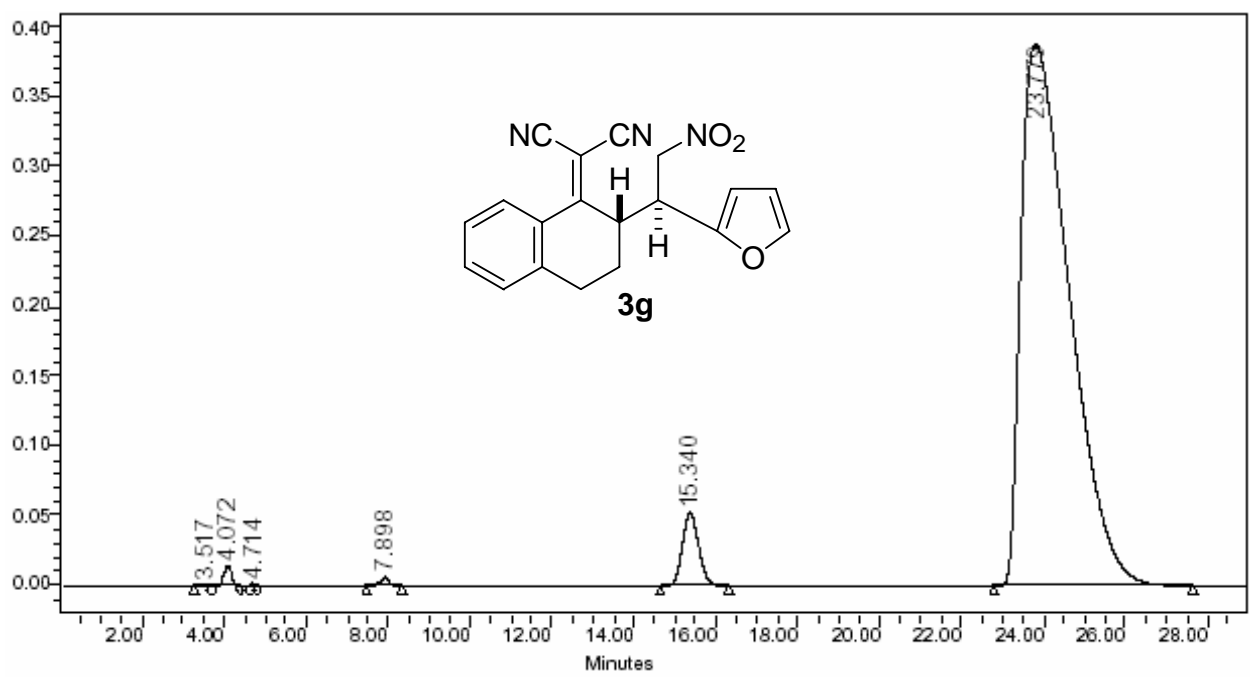

\begin{tabular}{|r|r|r|r|r|r|}
\hline & $\begin{array}{c}\text { RT } \\
(\mathrm{min})\end{array}$ & $\begin{array}{c}\text { Area } \\
\left(\mathrm{V}^{*} \mathrm{sec}\right)\end{array}$ & $\%$ Area & $\begin{array}{c}\text { Height } \\
(\mathrm{V})\end{array}$ & $\begin{array}{c}\% \\
\text { Height }\end{array}$ \\
\hline 1 & 3.517 & 14153 & 0.04 & 1015 & 0.22 \\
\hline 2 & 4.072 & 193990 & 0.58 & 14822 & 3.19 \\
\hline 3 & 4.714 & 8288 & 0.02 & 1281 & 0.28 \\
\hline 4 & 7.898 & 101973 & 0.30 & 6658 & 1.43 \\
\hline 5 & 15.340 & 1487653 & 4.42 & 52285 & 11.24 \\
\hline 6 & 23.779 & 31853625 & 94.63 & 389067 & 83.65 \\
\hline
\end{tabular}




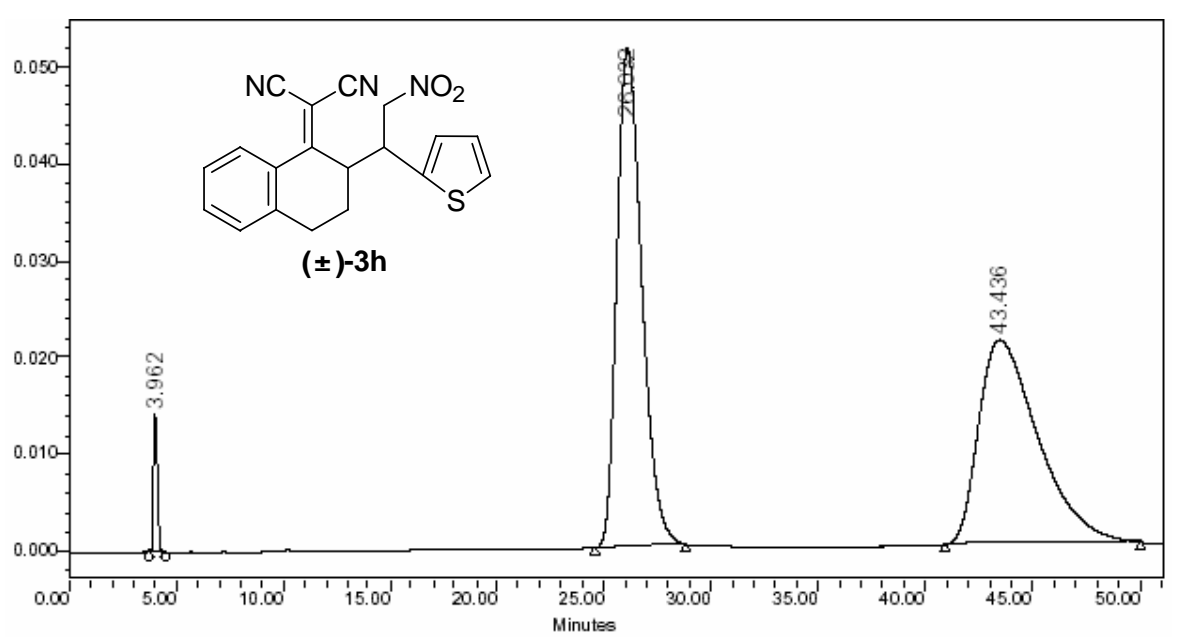

\begin{tabular}{|c|c|c|r|c|c|}
\hline & $\begin{array}{c}\text { RT } \\
(\text { min })\end{array}$ & $\begin{array}{c}\text { Area } \\
\left(V^{*} \text { sec }\right)\end{array}$ & $\%$ Area & $\begin{array}{c}\text { Height } \\
(\mathrm{V})\end{array}$ & $\begin{array}{c}\% \\
\text { Height }\end{array}$ \\
\hline 1 & 3.962 & 184092 & 2.19 & 14385 & 16.55 \\
\hline 2 & 26.032 & 4163035 & 49.43 & 51504 & 59.25 \\
\hline 3 & 43.436 & 4075716 & 48.39 & 21039 & 24.20 \\
\hline
\end{tabular}

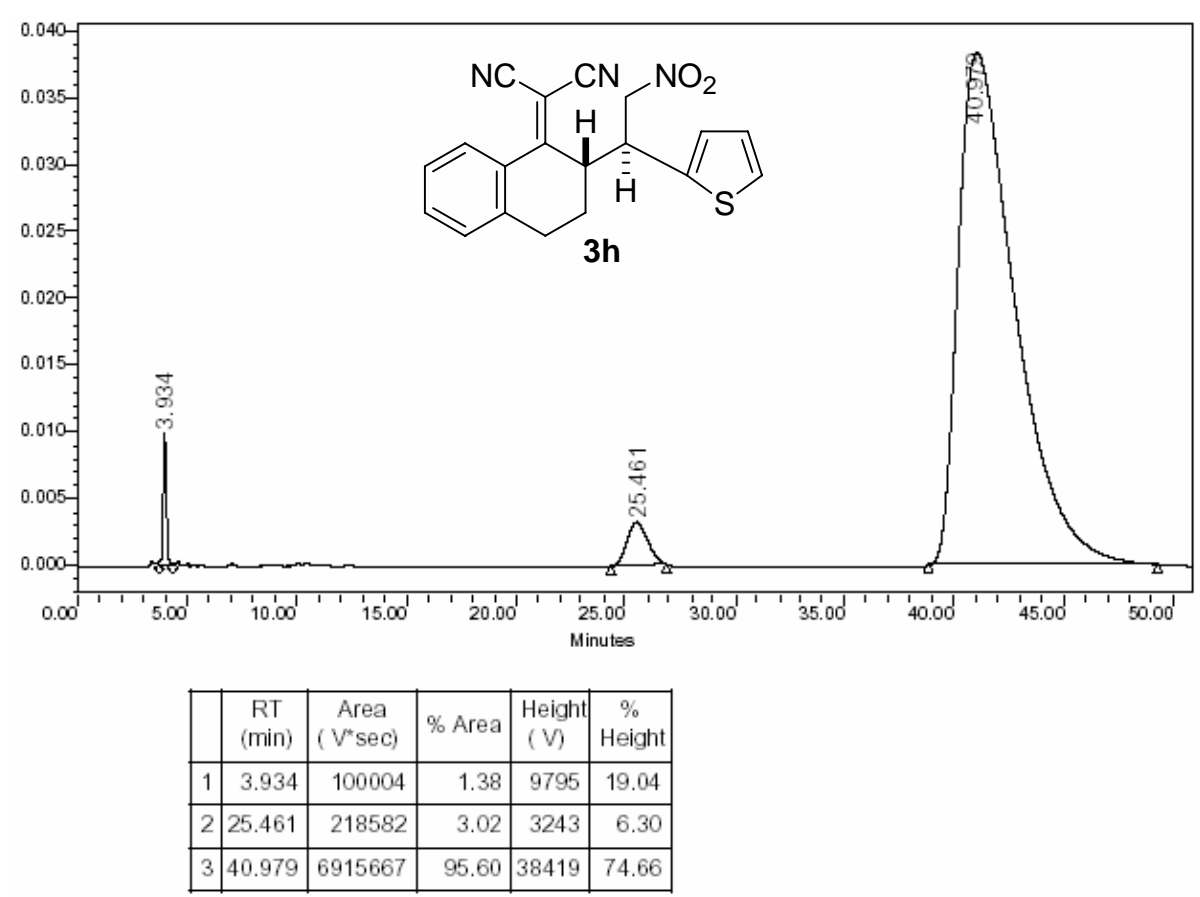




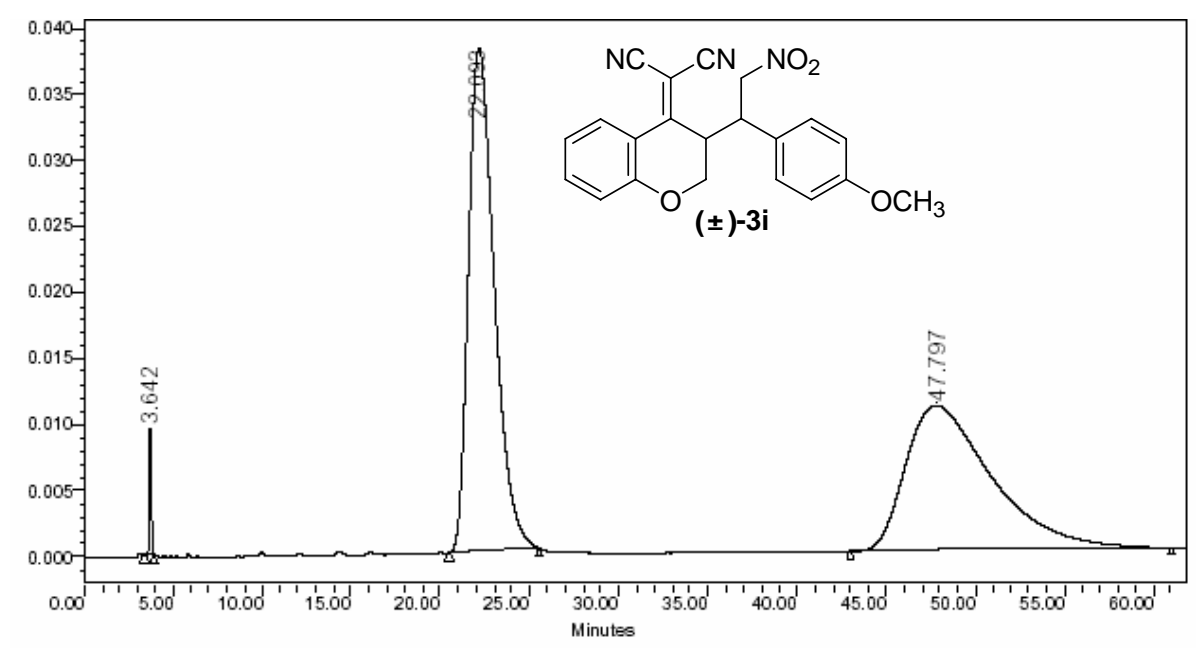

\begin{tabular}{|l|r|r|r|r|c|}
\hline & $\begin{array}{c}\text { RT } \\
(\mathrm{min})\end{array}$ & $\begin{array}{c}\text { Area } \\
\left(\mathrm{V}^{*} \text { sec }\right)\end{array}$ & \% Area & $\begin{array}{c}\text { Height } \\
(\mathrm{V})\end{array}$ & $\begin{array}{c}\% \\
\text { Height }\end{array}$ \\
\hline 1 & 3.642 & 84958 & 1.11 & 9641 & 16.40 \\
\hline 2 & 22.093 & 3856423 & 50.30 & 38113 & 64.85 \\
\hline 3 & 47.797 & 3725539 & 48.59 & 11017 & 18.75 \\
\hline
\end{tabular}
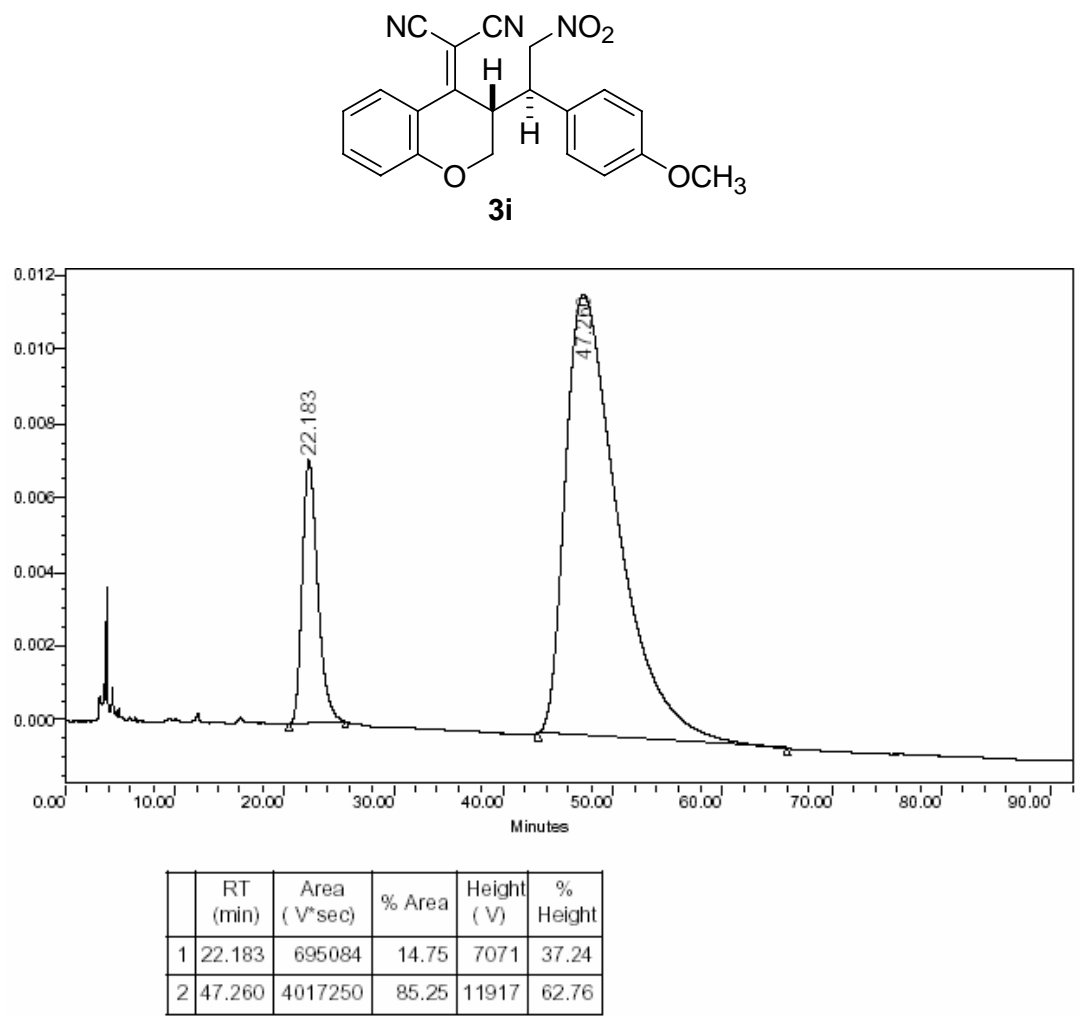

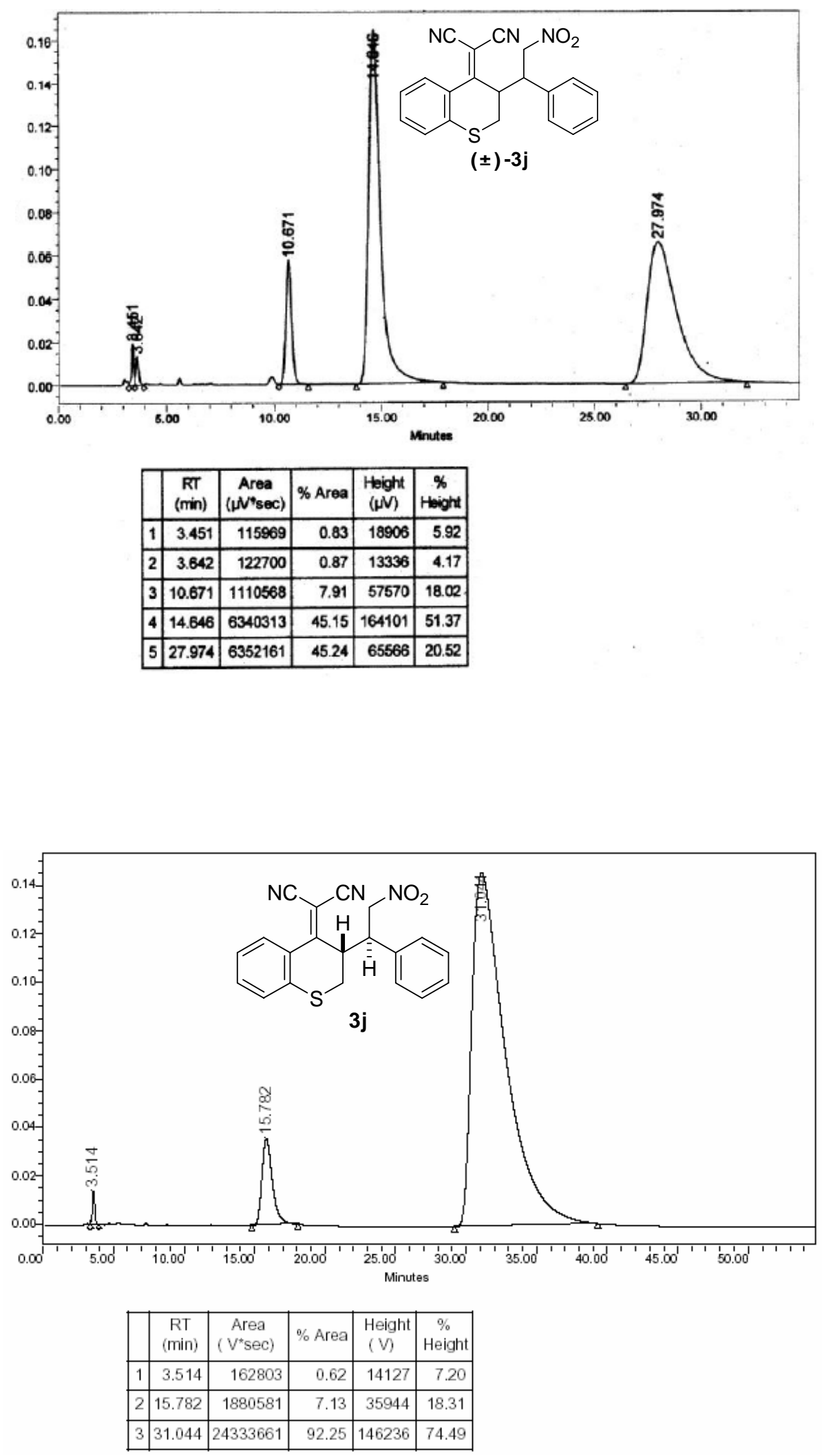

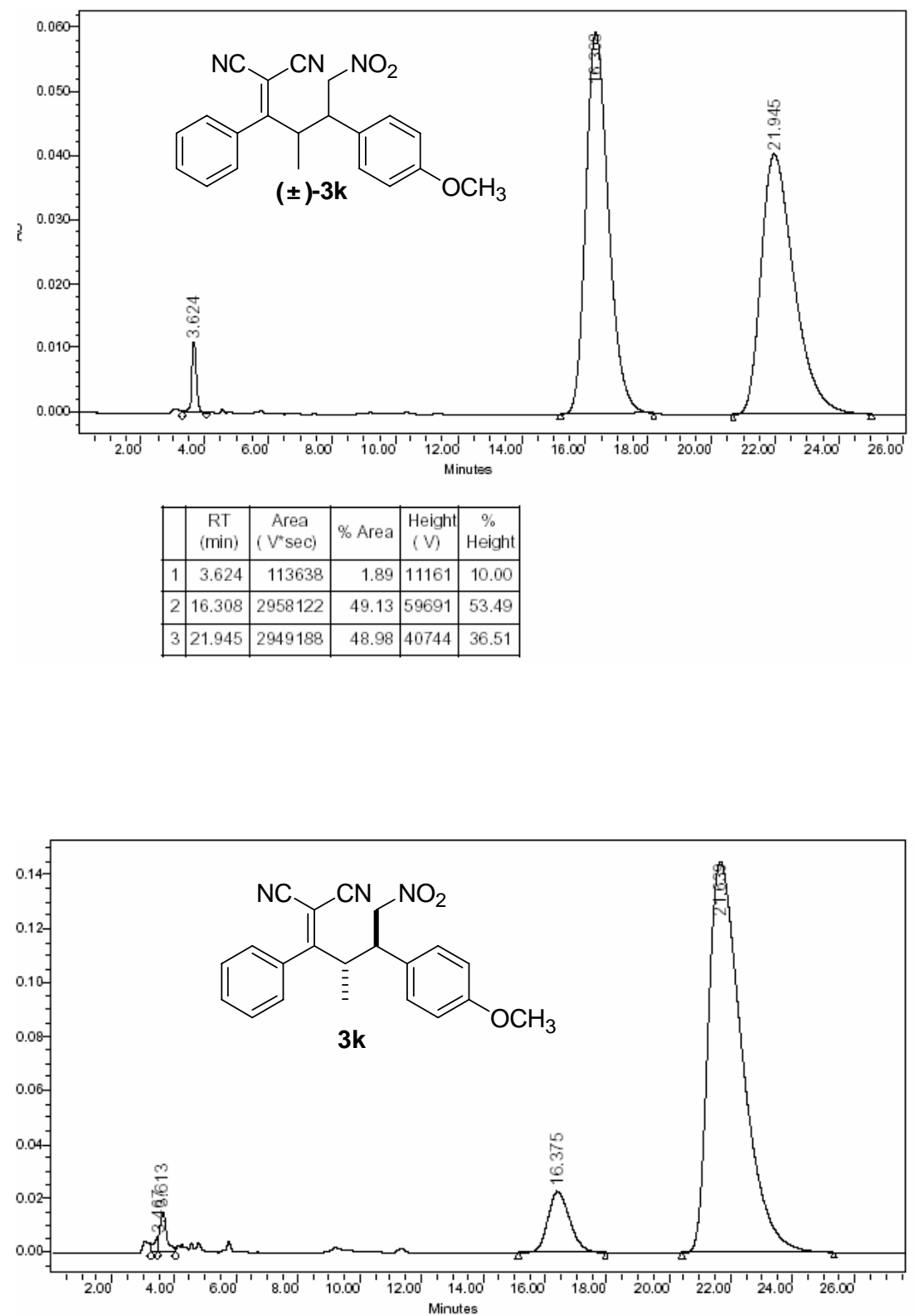

\begin{tabular}{|l|c|r|r|r|r|}
\hline & $\begin{array}{c}\text { RT } \\
(\mathrm{min})\end{array}$ & $\begin{array}{c}\text { Area } \\
(\mathrm{V} \text { (sec) }\end{array}$ & $\%$ Area & $\begin{array}{c}\text { Height } \\
(\mathrm{V})\end{array}$ & $\begin{array}{c}\% \\
\text { Height }\end{array}$ \\
\hline 1 & 3.467 & 59414 & 0.48 & 6208 & 3.28 \\
\hline 2 & 3.613 & 230773 & 1.86 & 15362 & 8.11 \\
\hline 3 & 16.375 & 1141217 & 9.18 & 22835 & 12.06 \\
\hline 4 & 21.639 & 11002348 & 88.49 & 145006 & 76.56 \\
\hline
\end{tabular}




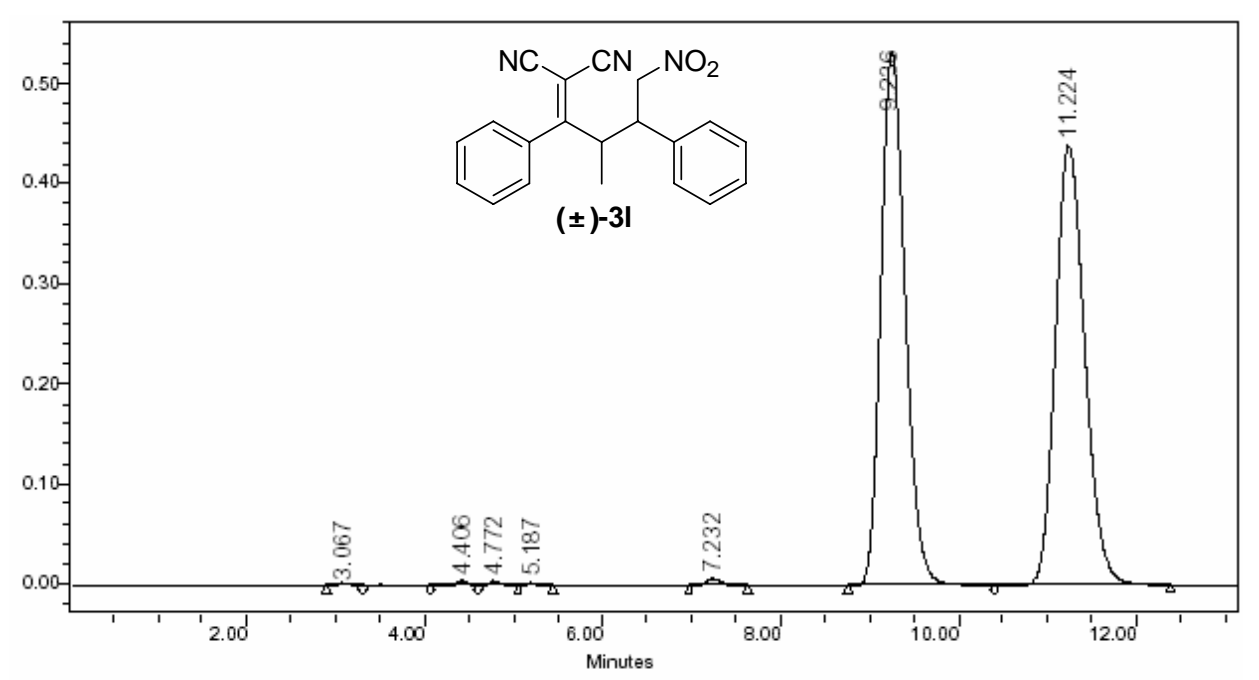

\begin{tabular}{|r|r|r|r|r|r|}
\hline & $\begin{array}{c}\text { RT } \\
(\mathrm{min})\end{array}$ & $\begin{array}{c}\text { Area } \\
\left(\mathrm{V}^{*} \mathrm{sec}\right)\end{array}$ & \% Area & $\begin{array}{c}\text { Height } \\
(\mathrm{V})\end{array}$ & $\begin{array}{c}\% \\
\text { Height }\end{array}$ \\
\hline 1 & 3.067 & 16661 & 0.08 & 1284 & 0.13 \\
\hline 2 & 4.406 & 41827 & 0.20 & 5075 & 0.51 \\
\hline 3 & 4.772 & 37441 & 0.18 & 4064 & 0.41 \\
\hline 4 & 5.187 & 21100 & 0.10 & 3122 & 0.31 \\
\hline 5 & 7.232 & 92681 & 0.45 & 7426 & 0.75 \\
\hline 6 & 9.236 & 10170041 & 49.60 & 534244 & 53.69 \\
\hline 7 & 11.224 & 10125505 & 49.38 & 439780 & 44.20 \\
\hline
\end{tabular}

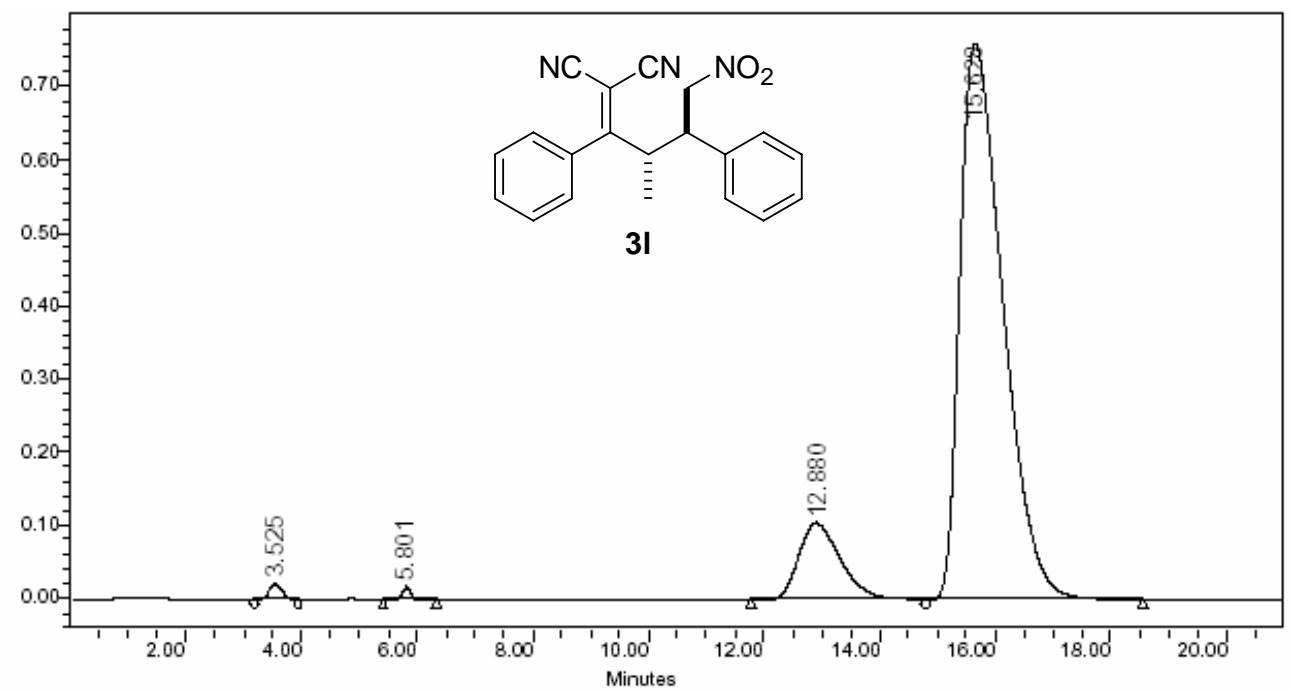

\begin{tabular}{|r|r|r|r|r|r|}
\hline & $\begin{array}{c}\text { RT } \\
(\text { min })\end{array}$ & $\begin{array}{c}\text { Area } \\
\left(V^{*} \text { sec }\right)\end{array}$ & \% Area & $\begin{array}{c}\text { Height } \\
(\mathrm{V})\end{array}$ & $\begin{array}{c}\% \\
\text { Height }\end{array}$ \\
\hline 1 & 3.525 & 377584 & 0.83 & 22252 & 2.46 \\
\hline 2 & 5.801 & 182509 & 0.40 & 17404 & 1.92 \\
\hline 3 & 12.880 & 5419023 & 11.97 & 105312 & 11.63 \\
\hline 4 & 15.628 & 39275491 & 86.79 & 760584 & 83.99 \\
\hline
\end{tabular}




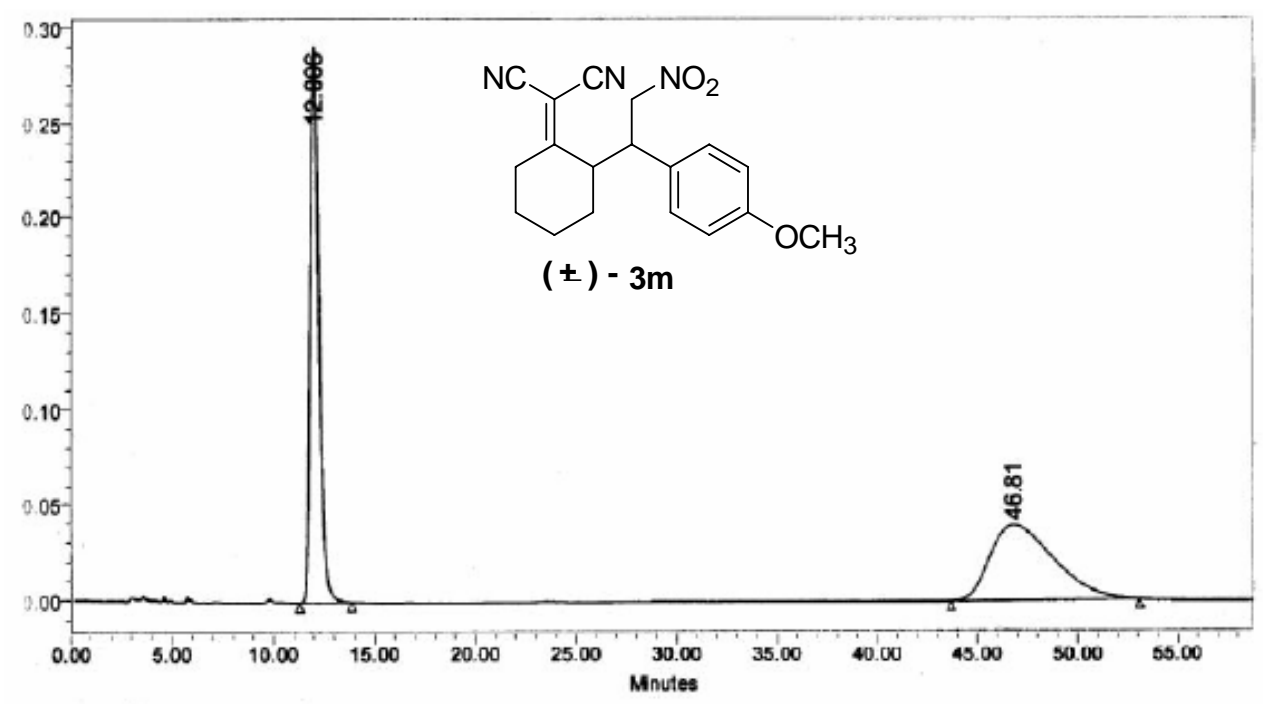

\begin{tabular}{|c|c|c|c|c|c|}
\hline & $\begin{array}{c}\text { RT } \\
(m i n)\end{array}$ & $\begin{array}{c}\text { Area } \\
(\mu \mathrm{N} * \mathrm{sec})\end{array}$ & \% Area & $\begin{array}{c}\text { Height } \\
(\mu \mathrm{N})\end{array}$ & $\begin{array}{c}\% \\
\text { Height }\end{array}$ \\
\hline 1 & 12.006 & 8909252 & 50.70 & 291085 & 88.11 \\
\hline 2 & 46.811 & 8664875 & 49.30 & 39270 & 11.89 \\
\hline
\end{tabular}

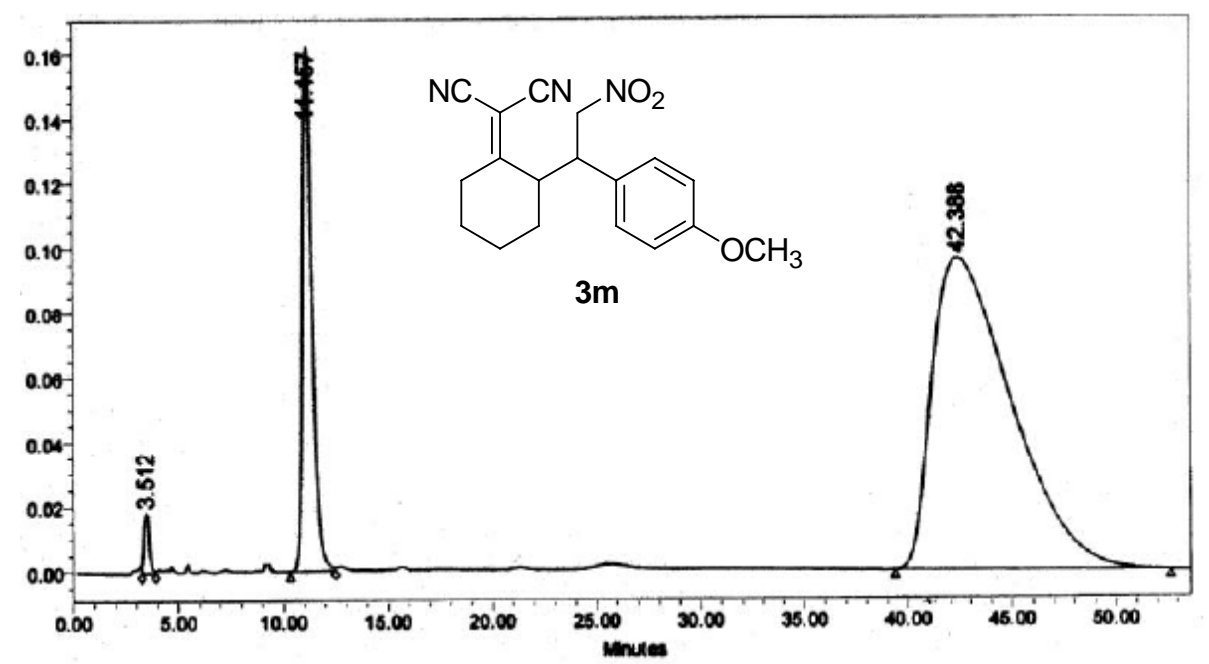

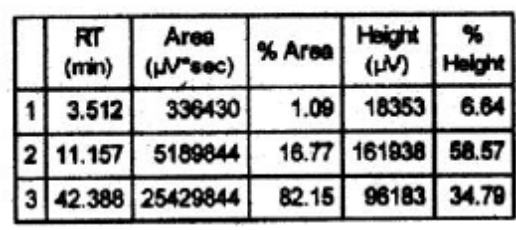

S46 

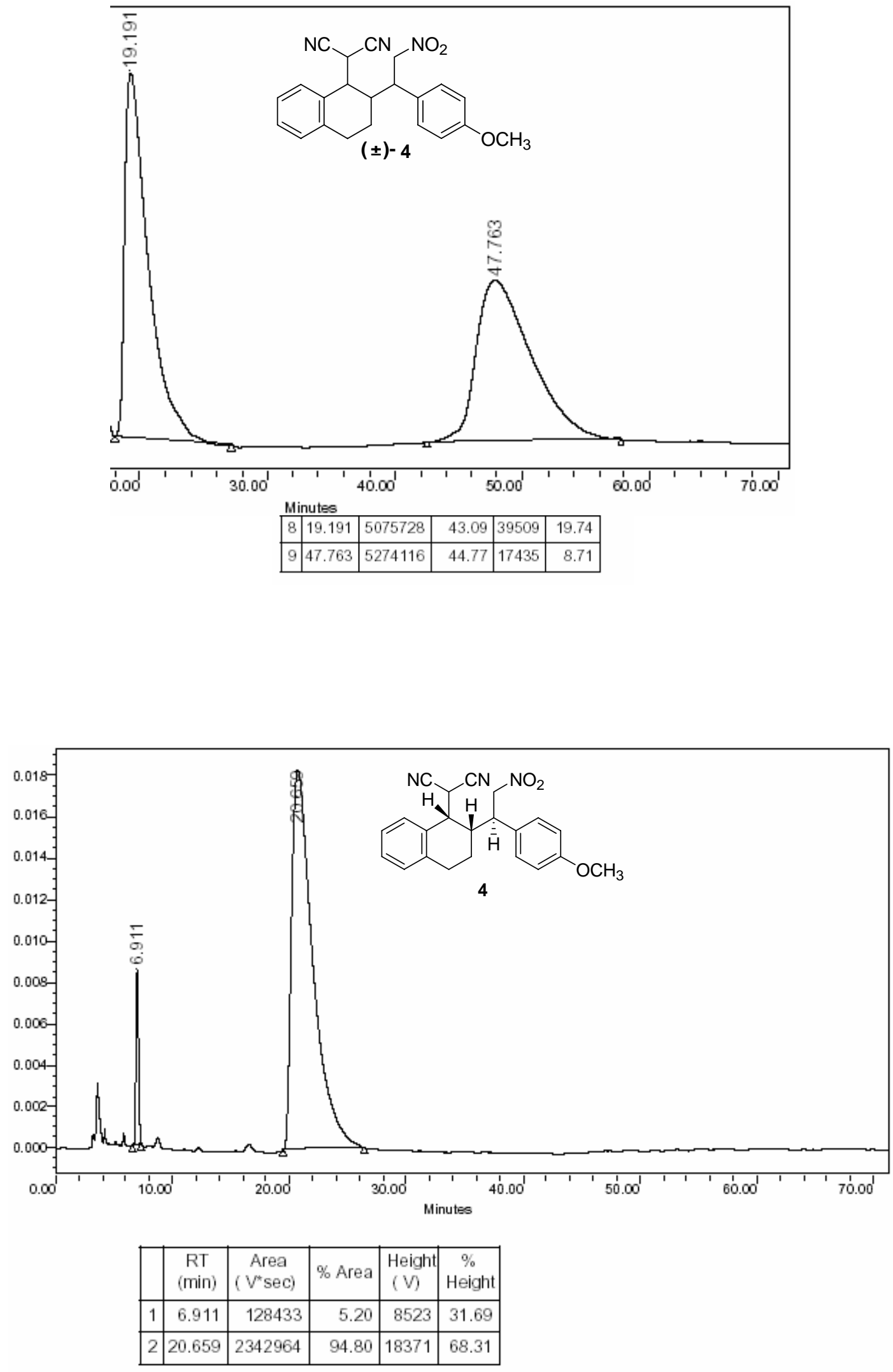

S47 


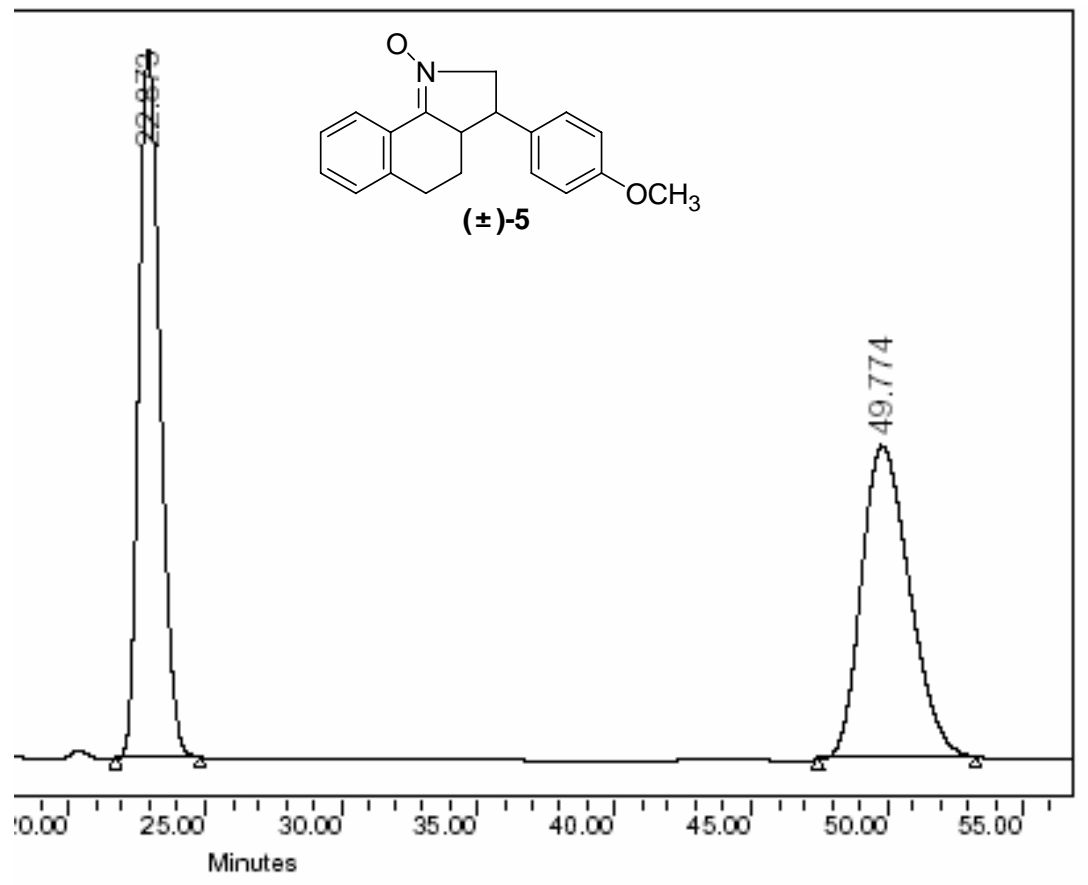

\begin{tabular}{|r|r|r|r|r|}
\hline 22.873 & 6762189 & 43.59 & 123670 & 45.18 \\
\hline 49.774 & 6712889 & 43.27 & 54656 & 19.97 \\
\hline
\end{tabular}

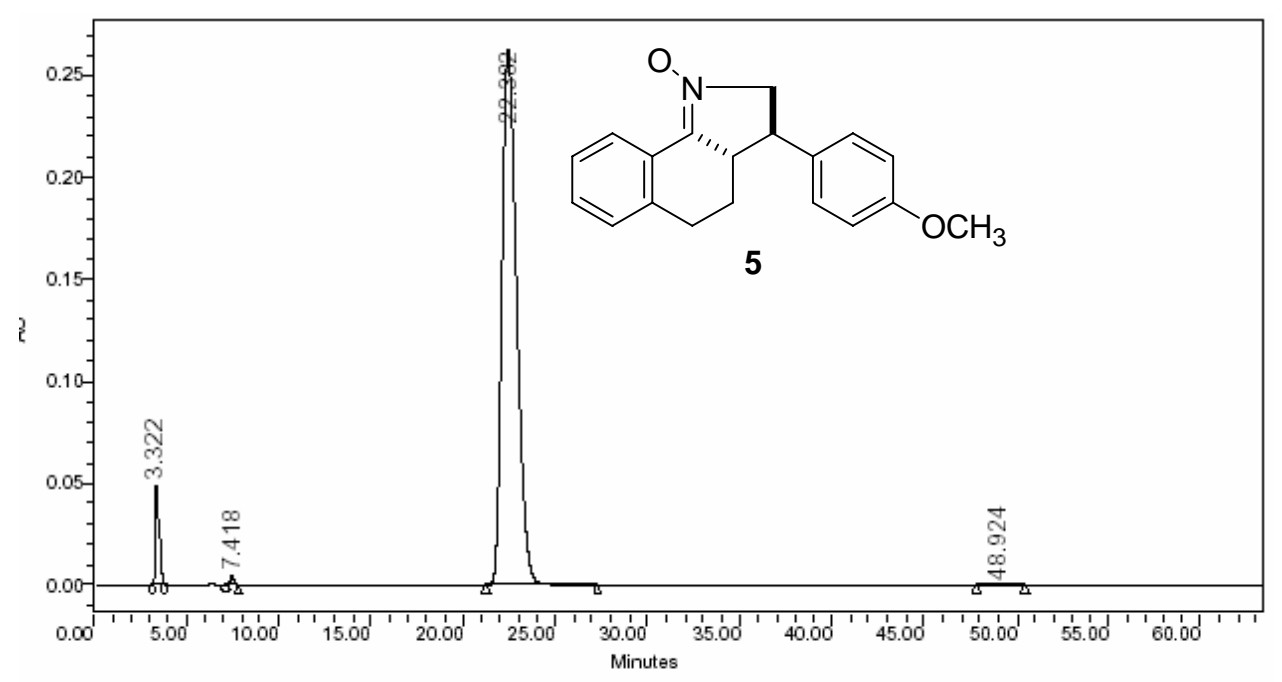

\begin{tabular}{|r|c|r|r|r|r|}
\hline & $\begin{array}{c}\text { RT } \\
(\mathrm{min})\end{array}$ & $\begin{array}{c}\text { Area } \\
\left(\mathrm{V}^{*} \mathrm{sec}\right)\end{array}$ & $\%$ Area & $\begin{array}{l}\text { Height } \\
(\mathrm{V})\end{array}$ & $\begin{array}{c}\% \\
\text { Height }\end{array}$ \\
\hline 1 & 3.322 & 742219 & 4.81 & 49307 & 15.55 \\
\hline 2 & 7.418 & 68905 & 0.45 & 4571 & 1.44 \\
\hline 3 & 22.382 & 14601402 & 94.69 & 263095 & 82.98 \\
\hline 4 & 48.924 & 7251 & 0.05 & 85 & 0.03 \\
\hline
\end{tabular}

